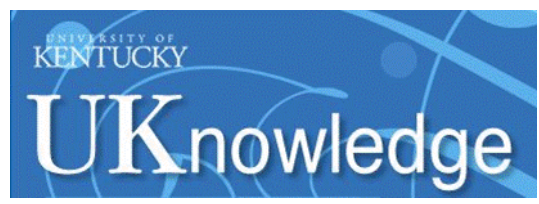

University of Kentucky

UKnowledge

Theses and Dissertations--Electrical and Computer Engineering

Electrical and Computer Engineering

2019

\title{
OPTIMAL DISTRIBUTION FEEDER RECONFIGURATION WITH DISTRIBUTED GENERATION USING INTELLIGENT TECHNIQUES
}

\author{
Ahmad Ghaweta \\ University of Kentucky, agh228@g.uky.edu \\ Digital Object Identifier: https://doi.org/10.13023/etd.2019.010
}

Right click to open a feedback form in a new tab to let us know how this document benefits you.

\author{
Recommended Citation \\ Ghaweta, Ahmad, "OPTIMAL DISTRIBUTION FEEDER RECONFIGURATION WITH DISTRIBUTED \\ GENERATION USING INTELLIGENT TECHNIQUES" (2019). Theses and Dissertations--Electrical and \\ Computer Engineering. 134. \\ https://uknowledge.uky.edu/ece_etds/134
}

This Doctoral Dissertation is brought to you for free and open access by the Electrical and Computer Engineering at UKnowledge. It has been accepted for inclusion in Theses and Dissertations--Electrical and Computer Engineering by an authorized administrator of UKnowledge. For more information, please contact UKnowledge@lsv.uky.edu. 


\section{STUDENT AGREEMENT:}

I represent that my thesis or dissertation and abstract are my original work. Proper attribution has been given to all outside sources. I understand that I am solely responsible for obtaining any needed copyright permissions. I have obtained needed written permission statement(s) from the owner(s) of each third-party copyrighted matter to be included in my work, allowing electronic distribution (if such use is not permitted by the fair use doctrine) which will be submitted to UKnowledge as Additional File.

I hereby grant to The University of Kentucky and its agents the irrevocable, non-exclusive, and royalty-free license to archive and make accessible my work in whole or in part in all forms of media, now or hereafter known. I agree that the document mentioned above may be made available immediately for worldwide access unless an embargo applies.

I retain all other ownership rights to the copyright of my work. I also retain the right to use in future works (such as articles or books) all or part of my work. I understand that I am free to register the copyright to my work.

\section{REVIEW, APPROVAL AND ACCEPTANCE}

The document mentioned above has been reviewed and accepted by the student's advisor, on behalf of the advisory committee, and by the Director of Graduate Studies (DGS), on behalf of the program; we verify that this is the final, approved version of the student's thesis including all changes required by the advisory committee. The undersigned agree to abide by the statements above.

Ahmad Ghaweta, Student

Dr. Yuan Liao, Major Professor

Dr. Aaron M. Cramer, Director of Graduate Studies 
OPTIMAL DISTRIBUTION FEEDER RECONFIGURATION WITH DISTRIBUTED GENERATION USING INTELLIGENT TECHNIQUES

DISSERTATION

A dissertation submitted in partial fulfillment of the requirements for the degree of Doctor of Philosophyin the College of Engineering at the University of Kentucky By

Ahmad Ghaweta

Lexington, Kentucky

Director: Dr. Yuan Liao, Professor of Electrical Engineering Lexington, Kentucky

2018

Copyright $^{(}$Ahmad Ghaweta 2018 
ABSTRACT OF DISSERTATION

\section{OPTIMAL DISTRIBUTION FEEDER RECONFIGURATION WITH DISTRIBUTED GENERATION USING INTELLIGENT TECHNIQUES}

Feeder reconfiguration is performed by changing the open/close status of two types of switches: normally open tie switches and normally closed sectionalizing switches. A whole feeder or part of a feeder may be served from another feeder by closing a tie switch linking the two while an appropriate sectionalizing switch must be opened to maintain the radial structure of the system. Feeder reconfiguration is mainly aiming to reduce the system overall power losses and improve voltage profile. In this dissertation, several approaches have been proposed to reconfigure the radial distribution networks including the potential impact of integrating Distributed Energy Resources (DER) into the grid. These approaches

provide a Fast-Genetic Algorithm "FGA" in which the size and convergence speed is improved compared to the conventional genetic algorithm. The size of the population matrix is also smaller because of the simple way of constructing the meshed network. Additionally, FGA deals with integer variable instead of a binary one, which makes FGA a unique method. The number of the mesh/loop is based on the number of tie switches in a particular network. The validity of the proposed FGA is investigated by comparing the obtained results with the one obtained from the most recent approaches. The second 
the approach is the implementation of the Differential Evolution (DE) algorithm. DE is a population-based method using three operators including crossover, mutation, and selection. It differs from GA in that genetic algorithms rely on crossover while DE relies on mutation. Mutation is based on the differences between randomly sampled pairs of solutions in the population. DE has three advantages: the ability to find the global optimal result regardless of the initial values, fast convergence, and requirement of a few control parameters. DE is a well-known and straightforward population-based probabilistic approach for comprehensive optimization.

In distribution systems, if a utility company has the right to control the location and size of distributed generations, then the location and size of DGs may be determined based on some optimization methods. This research provides a promising approach to finding the optimal size and location of the planned DER units using the proposed DE algorithm. DGs location is obtained using the sensitivity of power losses with respect to real power injection at each bus. Then the most sensitive bus is selected for installing the DG unit. Because the integration of the DG adds positive real power injections, the optimal location is the one with the most negative sensitivity in order to get the largest power loss reduction. Finally, after the location is specified, the proposed Differential Evolution Algorithm (DEA) is used to obtain the optimal size of the DG unit. Only the feasible solutions that satisfy all the constraints are considered.

The objective of installing DG units to the distribution network is to reduce the system losses and enhance the network voltage profile. Nowadays, these renewable DGs are required to equip with reactive power devices (such as static VAR compensators, capacitor banks, etc.), to provide reactive power as well as to control the voltage at their terminal bus. DGs have various technical benefits such as voltage profile improvement, relief in feeder loading, power loss minimization, stability improvement, and voltage deviation mitigation. The distributed generation may not achieve its full potential of benefits if placed at any random location in the system. It is necessary to investigate and determine the optimum location and size of the DG. Most distribution networks are radial in nature with limited short-circuit capacity. Therefore, there is a limit to which power can be injected into the distribution network without compromising the power quality and the system stability. This research is aiming to investigate this by applying DG technologies to the 
grid and keeping the system voltage within a defined boundary $[0.95-1.05$ p.u]. The requirements specified in IEEE Standard 1547 are considered.

This research considers four objectives related to minimization of the system power loss, minimization of the deviations of the nodes voltage, minimization of branch current constraint violation, and minimization of feeder's currents imbalance. The research formulates the problem as a multi-objective problem. The effectiveness of the proposed methods is demonstrated on different revised IEEE test systems including 16 and 33-bus radial distribution system.

KEYWORDS: network reconfiguration, Genetic Algorithm (GA), Differential Evolution Algorithm (DEA), Distributed Generation (DG), Sensitivity Analysis, Optimal Location and Size of DGs units.

Ahmad Ghaweta

2018

Date 


\title{
OPTIMAL DISTRIBUTION FEEDER RECONFIGURATION WITH DISTRIBUTED GENERATION USING INTELLIGENT TECHNIQUES
}

\author{
By
}

Ahmad Ghaweta

Dr. Yuan Liao

Director of Dissertation

Dr. Aaron M. Cramer

Director of Graduate Studies

Date 
This Dissertation is dedicated to my parents, my family, my brothers and sisters, my friend and to all who taught me. 


\section{Acknowledgments}

I am heartily thankful to my advisor, Dr. Yuan Liao, whose encouragement, guidance, and support from the initial stage to the final stage, helped me develop an understanding of the subject and complete the research.

Special thanks go to Dr. Paul Dolloff, Dr. Yuming Zhang, and Dr. Fazleena Badurdeen, who are on my dissertation committee, for their time in reviewing and evaluating this dissertation as well as for their guidance on my research. I also wish to thank my outside examiner Dr. Zhongwei Shen for his valuable time.

I would like to thank my wife for her encouragement and support through my PhD study. I wish to thank my entire extended family for providing a loving environment for me. My brothers, my sisters, my uncles, and my cousins were particularly supportive.

My deepest gratitude is due to my parents, Bashir Ghaweta and Mona Alhadi. They bore me, raised me, supported me, taught me, and loved me. I dedicate this dissertation to them. Lastly, I offer my regards and blessings to all of those who supported me in any respect during the completion of the thesis. It is a pleasure to thank many people who made this dissertation possible. This work was supported by the Ministry of Higher Education and Scientific Research of Libya. Special thanks to my country for helping make it possible and true. 


\section{TABLE OF CONTENTS}

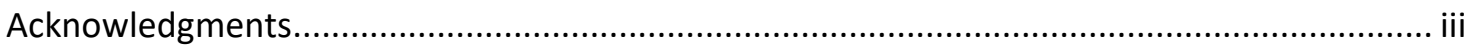

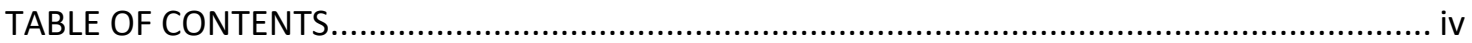

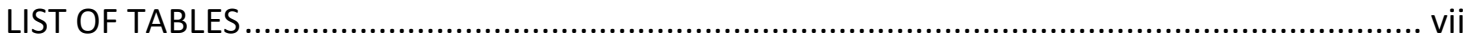

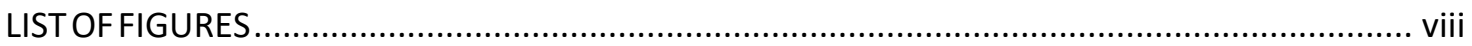

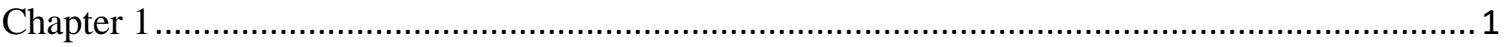

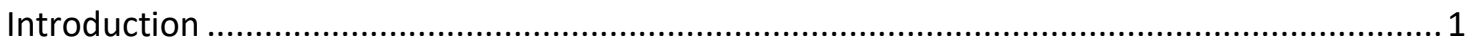

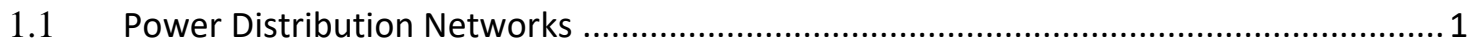

1.2 The impacts and benefits of integrating DERs in the distribution networks .................. 3

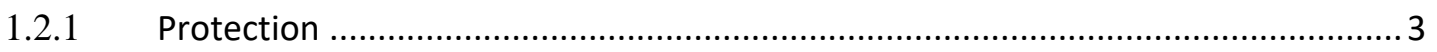

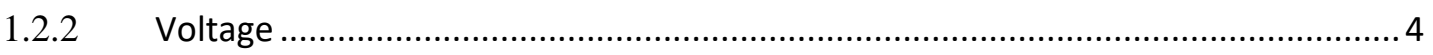

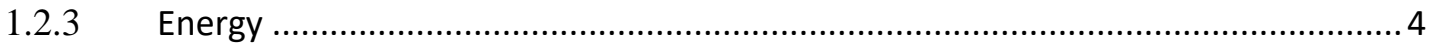

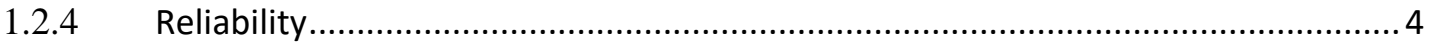

1.2.5 Capacity

1.3 Loss minimization of the Power Distribution system …............................................ 5

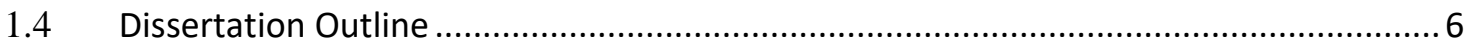

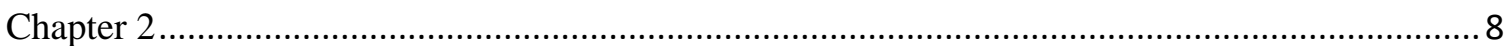

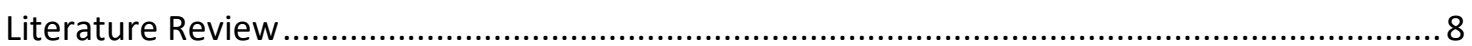

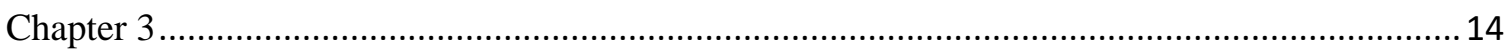

Distribution Feeder Reconfiguration For Loss Reduction ................................................ 14

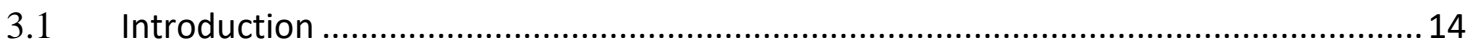

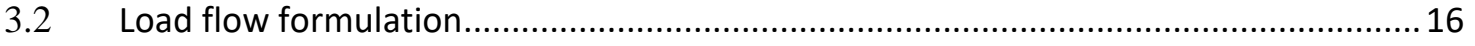

3.3 Power system modeling using graph theory ........................................................ 17

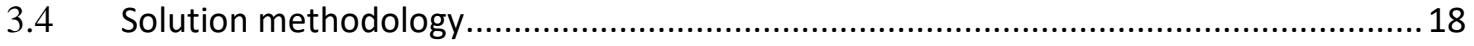

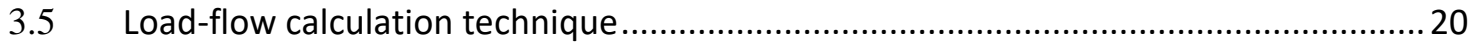

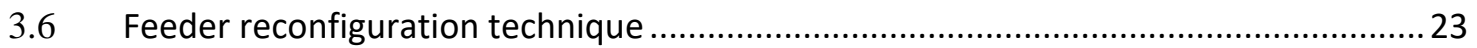

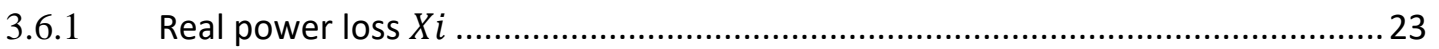

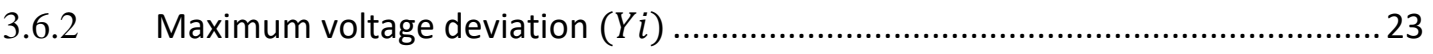

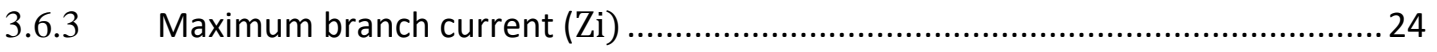

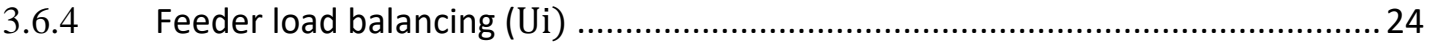

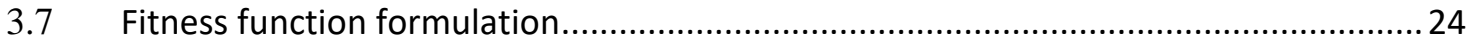

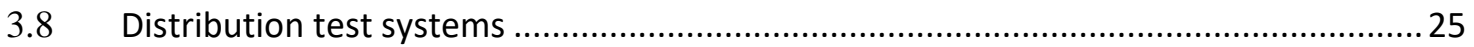




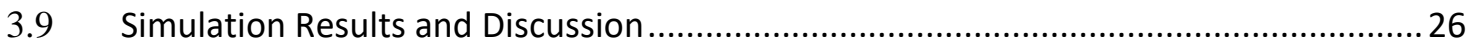

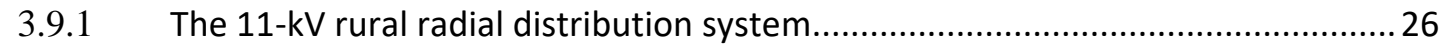

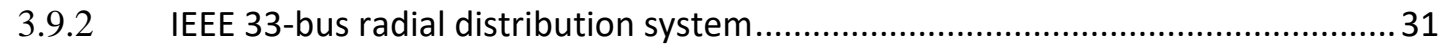

3.10 Test feeder performance after reconfiguration with the presence of DGs units..........34

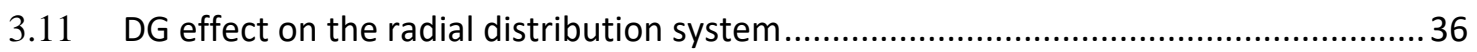

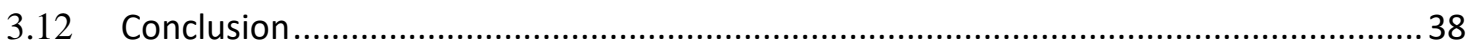

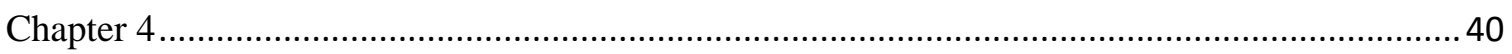

Distribution Feeder Reconfiguration for Power Loss Reduction Using Fast Genetic Algorithm

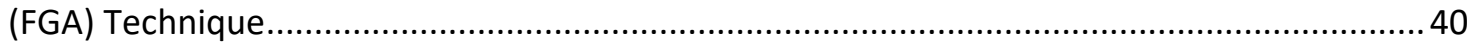

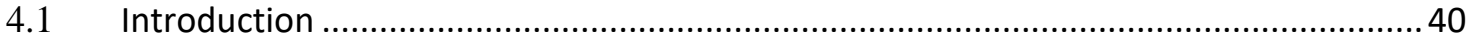

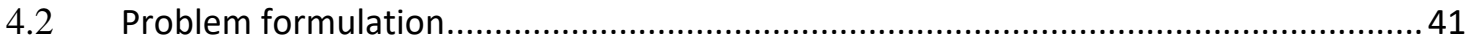

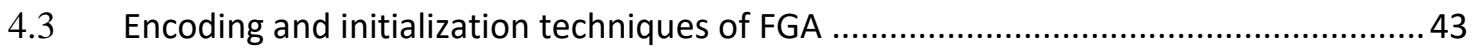

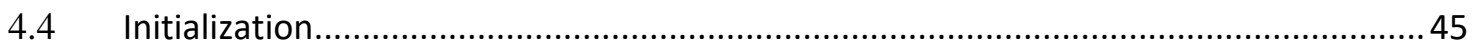

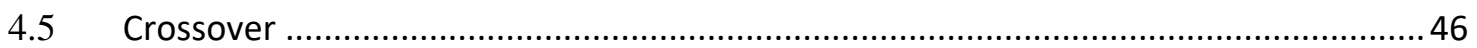

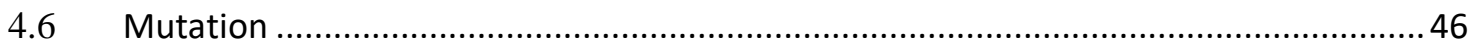

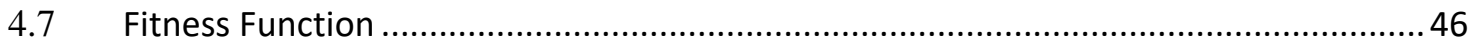

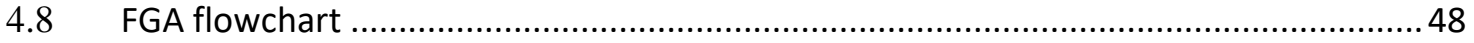

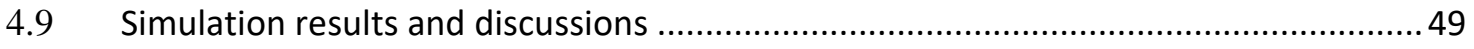

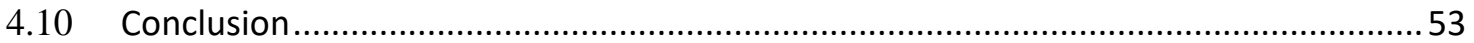

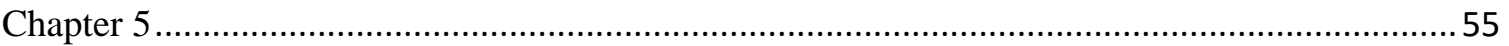

Optimal Distribution Feeder Reconfiguration with Distributed Generation using Differential

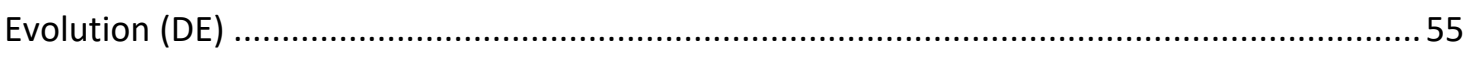

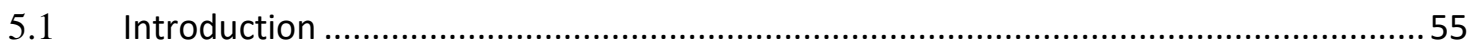

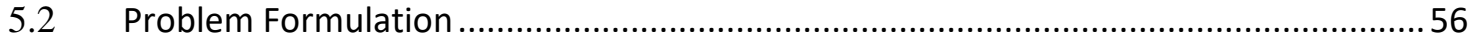

5.3 The Proposed Differential Evolution Algorithm (DEA) ............................................... 59

5.3.1 Overview of the optimization parameters........................................................... 59

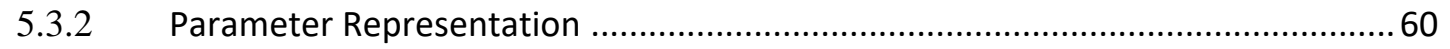

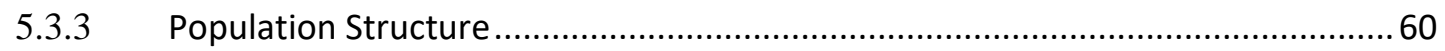

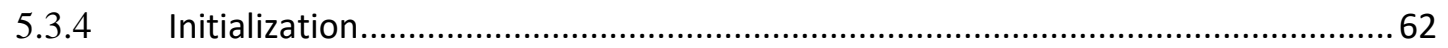

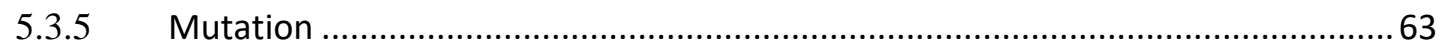

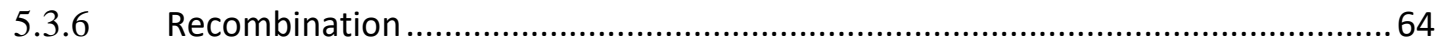

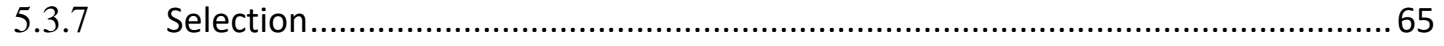

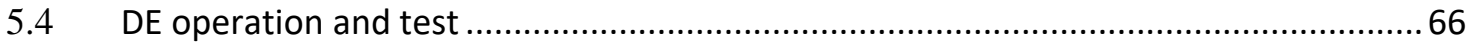




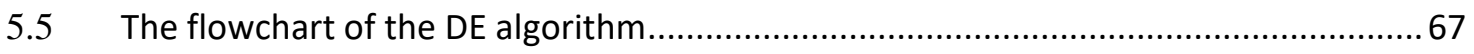

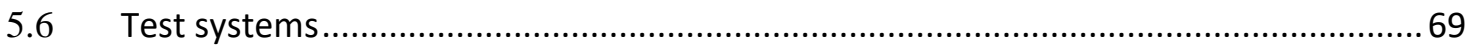

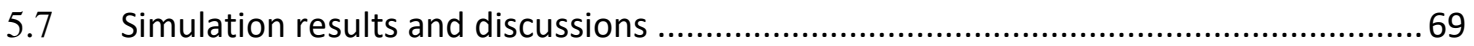

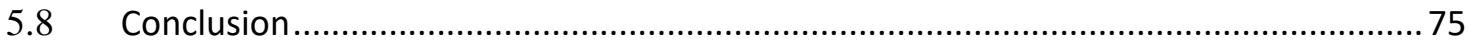

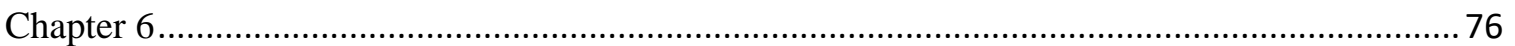

Optimal Placement and Sizing of Distributed Generation in a Radial Distribution System Using

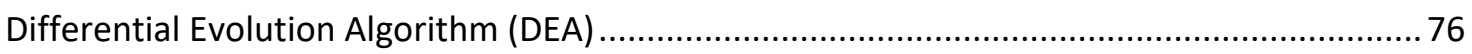

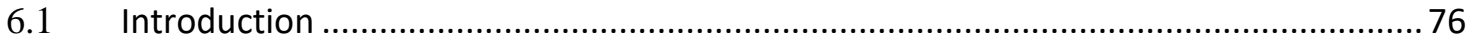

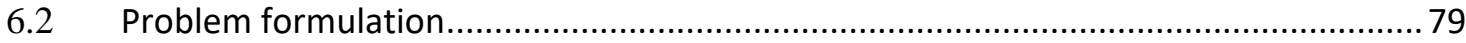

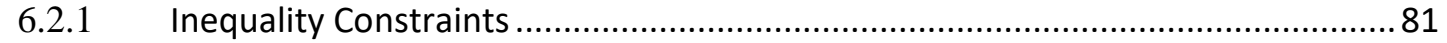

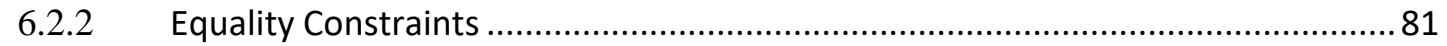

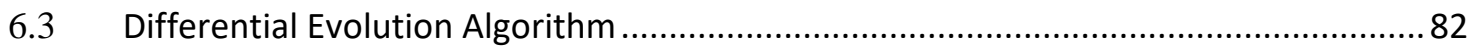

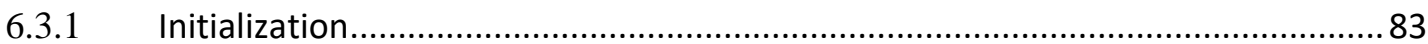

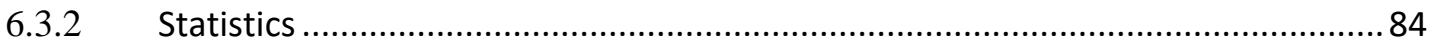

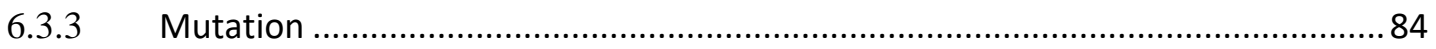

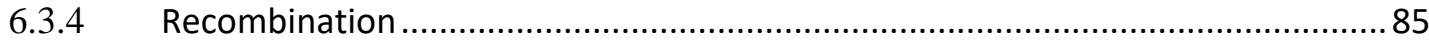

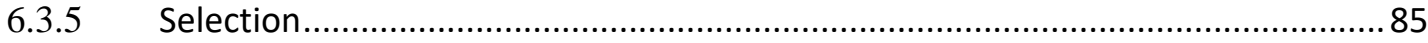

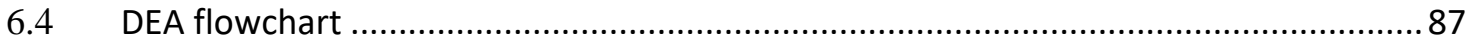

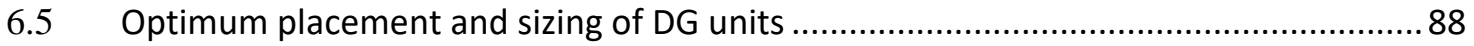

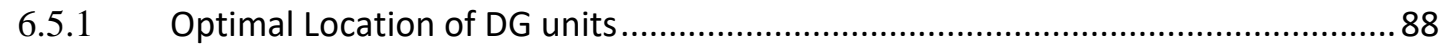

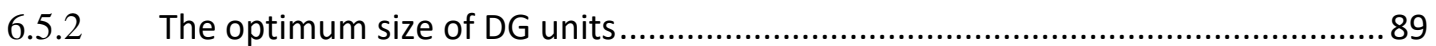

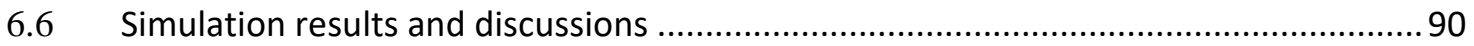

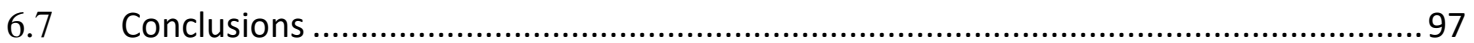

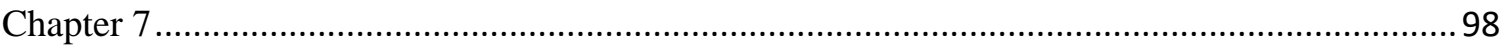

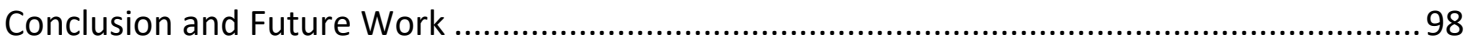

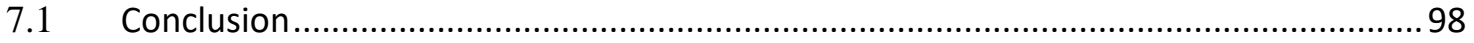

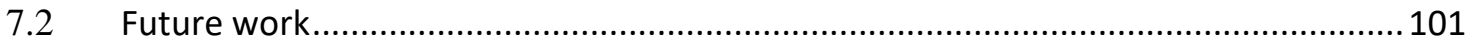

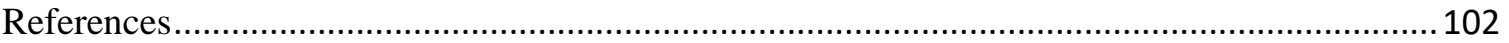

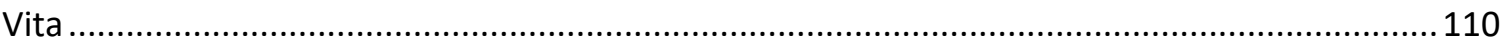




\section{LIST OF TABLES}

Table 3-1 Solution methodology of load flow analysis ........................................... 19

Table 3-2 load flow results before feeder reconfiguration......................................... 29

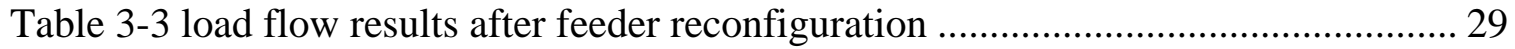

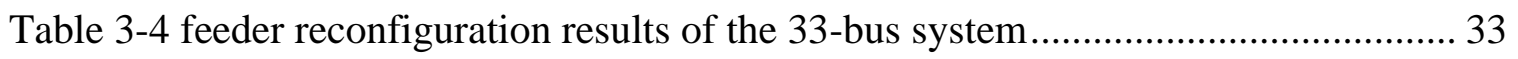

Table 3-5 Different case studies of the DGs unit replacement .................................... 35

Table 3-6 The impact of the positive and negative output Q of the DG inverter ............ 38

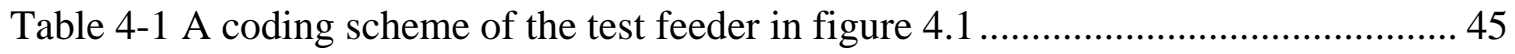

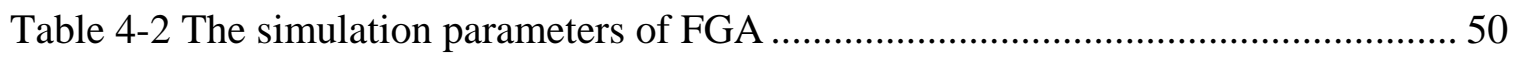

Table 4-3 The comparison of the proposed FGA with other methods ........................... 53

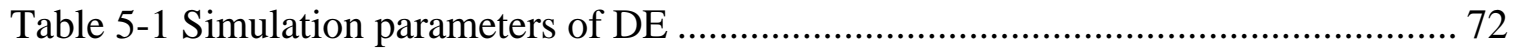

Table 5-2 Simulation Results of Both Test Systems Before and After Feeder

Reconfiguration and after reconfiguration using GA and proposed DE........................ 73

Table 5-3 Simulation Results of Both Test Systems After Feeder Reconfiguration Using

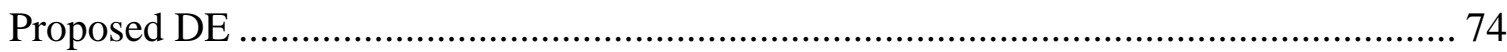

Table 6-1 Simulation results of the optimal switching plane and optimal placement and

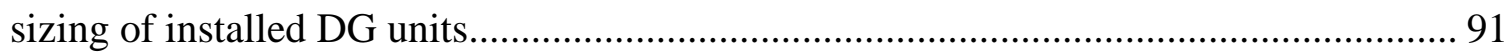

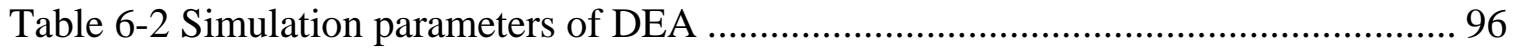




\section{LIST OF FIGURES}

Figure 3.1 Simple line diagram of a radial distribution system ..................................... 16

Figure 3.2 A graph representation of the power system ................................................. 18

Figure 3.3 Flow-chart for load-flow calculation of radial distribution network ............... 22

Figure 3.4 Distribution system with two substations and 11 ties switches before

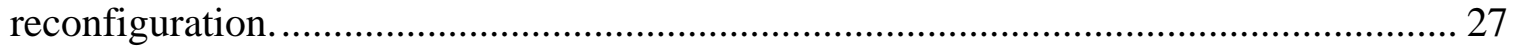

Figure 3.5 Distribution system performance after reconfiguration .................................. 30

Figure 3.6 Single line diagram of IEEE 33 bus distribution system................................ 31

Figure 3.7 loop formulation of the 33-bus test system ……………………………...... 32

Figure 3.8 Distribution system with tie-3 closed (case 1) ............................................ 33

Figure 3.9 Distribution system after reconfiguration case 2 (tie-5 operation).................. 34

Figure 4.1 Revised IEEE 16-bus test system ............................................................. 42

Figure 4.2 Flowchart of the proposed FGA .............................................................. 48

Figure 4.3 The proposed FGA performance characteristics of the best penalty value and

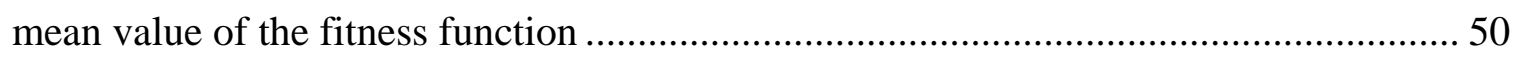

Figure 4.4 The real power loss computation using the proposed method ........................ 51

Figure 4.5 Zoomed in the plot for real power loss value ................................................ 52

Figure 4.6 Reactive power loss of the test system using FGA ……………………….... 52

Figure 5.1 Revised IEEE 16-bus test system ......................................................... 58

Figure 5.2 General Evolutionary Algorithm Procedure................................................... 62

Figure 5.3 Differential mutation: the weighted differential, $\mathrm{F}\left(\mathrm{x} \_(\mathrm{r} 1, \mathrm{~g})-\mathrm{x} \_(\mathrm{r} 2, \mathrm{~g})\right)$ is added

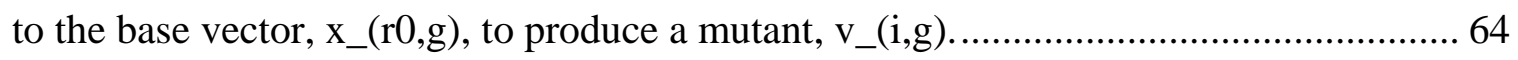

Figure 5.4 A flowchart of DE's generate-and-test loop................................................... 68 
Figure 5.5 Single line diagram of IEEE 33 bus distribution system, s denoted switch and

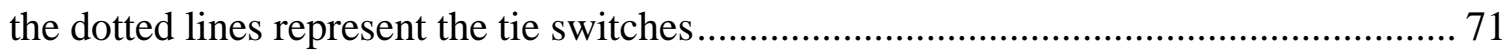

Figure 5.6 Convergence curve of the best fitness value of DE algorithm ..................... 72

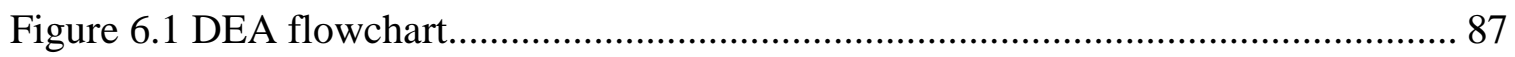

Figure 6.2 Loop representation of a single line diagram of IEEE 33-bus distribution system, where, s denoted switch and the dotted lines represent the tie switches ............ 90

Figure 6.3 Power Flow results with DG unit installed at bus \# 33 ............................... 92

Figure 6.4 Power Flow results with DG unit installed at bus \# 5 and 27 ...................... 93

Figure 6.5 Power Flow results with DG units installed at bus \# 19 and 33................... 93

Figure 6.6 Power flow results with DG units installed at bus \# 19, 33 and 2................ 94

Figure 6.7 Power flow results with DG units installed at bus \# 2, 23, 5 and 24............ 94

Figure 6.8 Power flow results with DG units installed at bus \# 33, 19, 23, 20 and 2...... 95

Figure 6.9 Power flow results with DG units installed at bus \# 23, 2, 24, 19, and 22..... 95

Figure 6.10 Convergence curve of the best fitness value of DE algorithm ................... 96 


\section{Chapter 1}

\section{Introduction}

\subsection{Power Distribution Networks}

Power Distribution networks are overgrowing and becoming more complex and complicated systems. Power generation is defined as the process used to produce electricity, normally at a central power plant. The transmission term is the process of transporting electricity to the customers at high voltages. Distribution is defined as the process of transforming electricity to lower voltages and transporting it shorter distances to individual consumers [1].

The traditional power system is fundamentally the interconnection of different power system components, such as synchronous machines, power transformers, transmission lines, transmission substations, distribution lines, distribution substations, and different types of loads. They are placed far away from the power consumption area, and electric power is transmitted through long transmission lines. Nowadays, the distribution system is neither traditional nor complex system because it has become smart systems. A smart system is a modern form of the traditional power grid which provides a more secure, reliable and dependable electrical service. It is, in fact, two-way communication between the utility and the electricity consumer. In addition, the distribution term now is divided into two types. One-Way distribution in which the power can only be distributed from the central plant using traditional energy infrastructure. The second type is the two-way distribution, while power still provided from the essential power plant, in a smart system, power can bi-directional and flow back to the transmission lines from a secondary power provider. These providers can be small or large scale individuals who have access to alternative power sources, such as wind turbine or solar panels, that can send energy back into the grid. The smart grid is capable of providing data and information of all the events in real time. The components of a smart grid include smart meters, smart substations, smart appliances, and advanced synchrophasor technologies. The utilities have the least controllability of the customer connected devices which is also where most of the change is occurring. The result is a set of challenges associated with further integrating of 
Distributed Energy Resource (DERs). To meet these challenges, an integrated approach for planning DERs is needed. In the late years, there has been a notable influx of DERs onto the grid particularly on the distribution system, either at the medium or low-voltage level. Specifically, installations of the solar photovoltaic (PV) systems are growing rapidly. PV systems are known as distributed generators (DGs) and also defined as an on-site generation or a decentralized energy source. Nowadays, there are a massive amount of the connected DGs units onto the grid. This shift alters the manner in which electricity is being generated, transmitted, and managed, thus necessitating a change in how utilities plan and integrate this resource. DERs conflicts have already risen between distribution systems designed for one-way power flow and DERs that want to force power flow in the opposite direction. Screening methods exist to avoid adverse impacts due to DERs, but this addressing the abundance of DERs interconnection requests and can result in higher overall costs if the resource is not fully integrated and located appropriately. For instance, let's consider designing the voltage regulator for a feeder. Without DERs, the network planner designs for a voltage drop from the substation to the feeder extremities. If the voltage is projected to drop too low during the peak demand periods, a capacitor bank or voltage regulator is added to boost the voltage level. Feeder voltage control is designed to yield voltages within ANSI C84.1. For the grid with DERs, the planner must also design for the voltage rise resulting from DER power output and must consider time(e.g., the impact of solar generation (at the bigging of the day or during the day)) in the analysis. An example of the time and location is the evaluation of PVs on a distribution feeder. Therefore, DERs are required to coordinate and approve when the DER are allowed to actively participate in regulating the voltage by changes its real and reactive output power. Besides, DERs must be coordinated with the protection devices when connected to the grid. A meshed system may better support DERs but requires an entirely different planning paradigm for distribution. New types of line equipment would be required to protect a new system configuration. Since the grid is becoming more complex, utility planning needs to change also they need a new planning method that can accommodate the more integrated system. While the planning functions of the DERs do not change, additional critical items should be considered to integrate DERs better and to quantify the overall impact of such sources more precisely. The main factors that need to be considered in a proactive planning 
approach with DERs are the size and location, distribution systems response characteristics, and DER technologies. Part of this research is aiming to optimally determine the location of the planned DERs using Differential Evolution Algorithm DEA. The distribution feeder response characteristics attentional is to determine the hosting capacity of the feeder. The hosting capacity of a feeder is defined as the amount of DERs a feeder can support under its existing topology, configuration, and physical response characteristics. If the hosting capacity is appropriately done then it will provide a range of information such as, how many DERs can be accommodated without system upgrades, what issues arise at the hosting capacity limits, the location of the DERs so that problems can be avoided, and the location where additional DERs are likely to cause issues on the grid. The third crucial item of integrating the DERs is the DER technologies. Different generation such as solar and wind can have widely varying impacts on voltage and capacity value compared with the dispatchable generation. The difference primarily comes from the timing in which the electricity is generated and the character of the energy output.

Distribution system assessment for DERs must focus on incorporating DERs while maintaining established standards of reliability and power quality. When planning a distribution system the first step to be considered is to establish the distribution feeder 's ability to host DERs that is to determine its hosting capacity. Hosting capacity is to determine the number of DERs that feeder can accommodate under current grid conditions without affecting power quality or reliability.

\subsection{The impacts and benefits of integrating DERs in the distribution networks}

\subsubsection{Protection}

The utility must retain the ability to detect and isolate faults as well as provide service restoration to all customers in a timely fashion. Additional DERs can affect the utility's ability to perform these functions. Besides, DERs must stop injecting real power when the grid lost its energy. In other words, DERs should inject power only when the connection point or bus to the grid is energized. Standard fault current analysis can be used to compare the fault response with and without the DERs to evaluate the potential impact on system protection. 


\subsubsection{Voltage}

Distribution system designed to maintain primary voltages level within the standard ranges. Typically, the voltage levels are kept within $\pm 5 \%$ of the normal rating. DER installations have the potential to change the voltage along a distribution feeder because of the power they inject into the grid. For variable generation such as wind and PVs, unacceptable voltages can occur, causing overvoltages or voltage deviations that can affect regulation equipment. Alternatively, if DERs coordinated with utility regulation, additional voltage support can be achieved. DERs must obey the IEEE1547 standard in which they must be coordinated with the utility operator and have the ability to participate and adjust their output power.

\subsubsection{Energy}

DER installation has the potential impact the reduce distribution losses because of the generated energy is provided closer to the consumer. DER is also known as an onsite generation source. The optimal location and size of the DERs is the crucial parameter of reducing system losses. The extent to which DERs can reduce losses depends on the location and the length of time for which the energy is injected into the grid. Briefly, DERs has a significant impact in reducing system losses if integrated at their optimal location. This research provides a promising method to determine the location and size of the DERs optimally.

\subsubsection{Reliability}

Reliability is a measure of the number and duration of interruptions of electrical service experienced by consumers. Additional, it is the ability of the system and its components to withstand instability. DER installation has the potential to improve reliability, but they must use reliable technologies and site in locations on the distribution system where they can effectively deliver power during system failure events and after fault isolation. As the size and capacity, DER output must be available at the time of need to improve system reliability. 


\subsubsection{Capacity}

Distribution systems are designed to provide service to all customers, especially at the peak demand periods when assets are most constrained. A potential benefit of integrating DERs into the distribution system is their ability to reduce net feeder demand and relieve capacity on existing distribution infrastructure, potentially deferring distribution-capacity upgrades. DERs must have the ability to provide capacity during the peak load periods. Capacity analysis requires specific information regarding thermal characteristics, the associated load profile characteristics, and projected load growth. Capacity is analyzed separately for each feeder and substation to identify the potential benefits arising from power being generated locally, as well as any adverse consequences of two-way power flows on feeder carrying capacity.

\subsection{Loss minimization of the Power Distribution system}

The amount of power losses in the electric distribution system and where they largely occur in the system are of great interest to the engineers in developing a rate structure for different classes of customers. Resistive line losses are also lower on higher-voltage systems, especially in a voltage-limited circuit. Because system losses are a function of the current squared $\left(I^{2} R\right)$, most losses occur on the primary near the substation. Losses occur regardless of the power factor of the circuit. Reducing the reactive portion of the total current, will significantly impact the system total losses.

Electrical power losses in distribution systems vary with numerous factors depending on system configuration, such as the level of losses through transmission and distribution lines, transformers, capacitors, insulators, e.g. [2]. In distribution systems, there are two types of Power losses, real power loss (active power $P$ ), and reactive power loss (reactive power $Q$ ). The real power loss is caused by the resistance of lines, while reactive elements produce the reactive power loss.

Traditionally, there are different ways to reduce system losses including using a higher system voltage, reduce loads, balance the circuits, increase power factor, use three-phase circuits, and use larger size conductors. Additional, Capacitors provide enormous benefits to distribution system interpretation. Such as, capacitors reduce system losses, free up capacity (the same circuit can serve more load), and reduce voltage drop. This research is 
aiming to reduce total system losses (reduce line current) by using intelligent techniques such as distribution feeder reconfiguration, integrating DERs in the system to mitigate the amount of energy provided by the central power plant ( onsite generation ), and using DE algorithm to reconfigure the network.

\subsection{Dissertation Outline}

The optimization approach is a procedure in which the best possible values of decision variables are acquired under a certain set of conditions/constraints and in accordance with a selected optimization fitness or objective function. The most common and known optimization algorithm applies to a design that will minimize the total cost function or maximize the reliability or any other particular objective.

Nowadays, there exist a lot of various optimization approaches that work using heuristicbased search techniques in deterministic and stochastic contexts and gradient-based. In order to achieve high applicability of the optimization approach to various problem domains, natural and physical principles are mimicked to develop robust optimization algorithms. Evolutionary algorithms, simulated annealing, ant colony optimization, memetic algorithms, particle swarm optimization are few examples of such algorithms.

The remainder of this dissertation is organized as follows: The literature review related to the feeder reconfiguration technique and the contribution of the DER's output power and its impact into the radial distribution networks. The feeder reconfiguration to solve the multi-objective problem using a new load flow strategy by employing a graph theory is described in Chapter 2. In chapter 3 the reconfiguration problem is solved by using the intelligent technique. Traditional GA was commonly used to solve this problem, then in the last decade many researchers tried to improve the performance of the GA. Since the distribution networks have become larger and larger and also because of regulation and the environmental concerns has brought to the attention of the energy market the need for clean energy resources. The central traditional power plants have to be upgraded and the inside power sources are needed such as DGs and offshore power plants. Now the need for a robust method to take into account the impact of the integrated DGs such impacts is the reverse power and voltage violation, etc,. Additionally, the need for a fast and quick approach to reconfiguring the system especially when there is a significant change in the 
load or when the fault occurred in the system. Chapter 4 provided the fast genetic algorithm (FGA) in which we consider all the operation requirements besides the constraint and promising to have fast convergence and less memory needed. After the study and the simulation in chapter 3 and because of the intense and deep research about the optimization techniques it comes to my mind to use the evolutionary algorithms specifically the Differential Evolution Algorithm (DEA). The evolutionary process, first developed to evolve finite state machines (FSM), consists of finding a set of optimal behaviors from a space of observable behaviors [3] [4]. In this chapter (chapter 4) I proposed to implement DE algorithms to solve the radial distribution systems. The impact of the integrated DGs in the system is considered and in fact, the size and location of DGs units are randomly chosen because the assumption is that these units already exist in the grid, therefore, it is not controllable. Now, what if the utility company for example wants to control these sources, or in other words wants to plane new off-site sources. These questions have brought the idea of chapter 6 in which the optimal location and sizing of the planned DGs using the proposed DEA. The proposed topology in chapter 6 is to reconfigure the system first, then obtained the location of the planned DGs unit by using the real power loss sensitivity analysis, and then after setting the DG at its obtained optimal location the size is computed by using DEA. Keeping in mind that the connection of the DGs units must obey the IEEE 1547 standard. Finally, the conclusions and future work are discussed in Chapter 7. 


\section{Chapter 2}

\section{Literature Review}

The existing distribution networks are growing in complexity, due to the gradual increase of power demand and the existence of different customers with more sensitive loads. Each feeder in a distribution system has a different mixture of commercial, residential and industrial type loads, and it is well known that the daily load variations of these load types are dissimilar. Consequently, the peak loads on substation transformers, on individual feeders, or on feeder sections occur at different times (non-coincidence of peaks). Feeder reconfiguration processer allows the transfer of loads from loaded feeders or transformers (heavily loaded) to relatively less loaded feeders or transformers (lightly loaded). Such transfers will effectively alter the level of loads on the feeders being switched and also improve the voltage profile of the feeders and reducing the overall system power losses [5]. Feeder reconfiguration is performed by changing the open/close status of switches. Primary distribution networks contain two types of switches, known as tie switches (normally open) and sectionalizing switches (normally closed). These switches are designed for both protection and configuration purposes. A whole feeder or part of a feeder may be served from another feeder by closing a tie switch linking the two while an appropriate sectionalizing switch must be opened to maintain the radial structure of the system.

The use of distribution feeder reconfiguration for loss reduction was first proposed by Merlin and Back (1975) [6]. They have used a branch-bound type optimization strategy to calculate the minimum loss configuration. Based on their method, a heuristic algorithm has been suggested by Shirmohammadi and Hong [7]. Their solution procedure also starts by closing all of the network switches to find the maximum voltage difference between the tie switches. Then to consider closing first the tie switch with the maximum voltage difference, after that form the loop and opening and closing each sectionalizing switch of the branches one after another to establish the optimal flow pattern in the system. Borozan [8] have presented a network reconfiguration technique similar to that of Shirmohammadi and Hong [7]. However, their methodology contains three main parts: real-time load estimation,

effective determination of power loss configuration, and cost/benefit evaluation. Civanlar 
[9] made the use of heuristics to determine a distribution system configuration which would reduce line losses. He used a branch exchange methodology for switching mechanism: to ensuring the radial nature of the distribution system opening of any switch was required the closure of another switch correspondingly. Baran and Wu (1989) [10] have attempted to improve the method of Civanlar by introducing two approximation formulas for power flow in the system loads transfer. Baran and Wu Power-flow equations were defined by a recursive approximation of $P, Q$, and $V$ at each bus. Then he proposed an efficient load flow equations to solve power flow equations by formulating the load balancing and loss reduction as an integer programming problem. Lu. [11] have proposed two algorithms minimizing the real power loss in distribution networks. Taylor and Lubekaman [12] have proposed a heuristic approach to distribution feeder reconfiguration for loss reduction, removal of transformer overloads, and feeder constraint problems. To find the global or near-global optimal solutions, Chiang and Jean-Jumean [13], [14] and Jeon et al. [15] have proposed new solution methodologies using the simulated annealing algorithm for the reconfiguration. Chen and Cho [16] have presented optimal switching criteria using binary integer programming with a branch-and-bound technique for network reconfiguration to achieve energy loss minimization for the short-term and long-term operation of distribution systems. Wagner et al. [17] have compared various methods of feeder reconfiguration for loss minimization problems. Zhou et al. [18] have proposed two feeder reconfiguration algorithms for the service restoration and load balancing purpose. Their method combined the optimization techniques with fuzzy logic and heuristic rules for robust performance and efficiency improvement. Zhou et al. [19] have also proposed another heuristic-based feeder reconfiguration algorithm for reducing the operating cost in the real-time operating environment. Lin and Chin [20] have presented an algorithm for distribution feeder reconfiguration using the ohmic index, a voltage index, and decision index to determine the switching operation. Borozan and Rajakovic [21] have considered the application aspects of optimal distribution network reconfiguration. Taleski [22] have provided a method to determine the configuration with minimum energy losses for a given period. $\mathrm{N}$. Rug and S. Siris [23] have presented an approach for finding the optimal implementation of feeder reconfiguration in unbalanced loading distribution systems with the objective of power loss reduction. M.P.sharma [24] also have presented an in-depth analysis of Feeder 
Reconfiguration (FR) of 139-bus, $33 \mathrm{kV}$ distribution system, by using minimum Power Flow-based Reduction (MPFR) technique.

Network reconfiguration problems have been considered as a crucial area of interest in recent years. Most of the approaches proposed heuristics, approximate techniques or mathematical programming. Therefore, the obtained results are either approximate or only local optimum solutions. Network reconfiguration using genetic algorithms (GA's) based approaches and simulated annealing have also been used by [25].

In general distribution feeder reconfiguration is implemented from time to time especially when there is a significant change in loads or sudden fault occurring on the network. Network reconfiguration is the method of changing the topological structure of feeders by changing the open/closed status of sectionalizing and tie switches. Nowadays the renewable energy resources have become a primary alternative energy source. These sources are also known as distributed generation (DGs) units. They are installed in the system to serve as an on-site source of power near the site where the energy is needed and to be consumed. DGs can be presented by different types of resources and technologies such as wind, solar, fuel cells, hydrogen, biomass, and combined heat and power (CHP). These technology has drawn significant attention for utilities to accommodate DG units in their systems [26] [27]. In a real system, the location of the existing DG units is not quite demonstrated by utility company because it is customer owned. Therefore, its location and size are not controllable, and if it is agreed to be integrated into the grid, then it becomes a type of fixed source.

On the other hand, if utility company owns it, then becomes controllable and its location and size may be determined based on some optimization methods such as sensitivity analyses as presented by Das [28] or Loparo [29], or any other methods. To date, there is no standardized regulation enacted to define the frequency of reconfiguring the distribution network. However, because distribution network applications use much real-time information, which usually has a discrete time step of an hour [30], distribution networks may be configured in each hour based on the status of time-varying loads, and available output power from the DG units [31] [32]. 
The genetic algorithm (GA) was first proposed by Nara [33] for distribution network loss minimization. Many other authors have followed and improved this method for the distribution network optimization.

Conventional genetic algorithms often have slow convergence speed and easily stuck at a local optimum. Thus, in order to overcome these shortcomings, many interested researchers have made considerable efforts to improve GA's performance of (Vasconcelos and Saldanha (1997) [34], Vasconcelos et al. (2001)) [35]. The significance of the probabilities of crossover $(p c)$ and mutation $(p m)$ in controlling GA's has been acknowledged in GA research, since $p c$ and pm greatly determine whether the algorithm will find a near optimum solution or whether it will find a solution efficiently (Zhang et al. (2004)) [36]. This research proposes a Fast Genetic Algorithm (FGA) for distribution feeder reconfiguration for loss reduction and load balancing with the ability to find the global solutions. It also introduces some new features improving accuracy and the computational efficiency, including a unique population generation, a Tabu list of infeasible solutions, a two-termination criterion and also the refined adaptation of crossover and mutation probabilities according to the genetic diversity in the population. Different case senarious will be considerd inhere such as, load flow analysis before abd after network configurtion and feeder reconfiguration with/without the DGs connection. Ramos et al. [37] developed algorithms based on genetic algorithm and conventional mixed integer linear problem. Wang et al. [38] proposed an algorithm to minimize loss and load balancing for a large-scale unbalanced system using network reconfiguration.

Now for the distributed generation DG implementation in distribution power system network, there are many effort research employed in this aspect. N. Rugthaicharoencheep and S. Sirisumrannukul [39] have presented a work to minimize system total power loss in the presence of DGs that cause reverse power flows and voltage variations. Their method focused on solving optimization problem subject to system constraint consisting of loadpoint voltage limits, radial configuration format, no load-point interruption, and current feeder capability limits, the idea in their research is using a Tabu search algorithm. Ding and Kenneth [29] they followed up on their previous work and have presented feeder reconfiguration for unbalanced distribution systems. They proposed a sensitivity analysis 
of the real power injection and nonlinear programming to determine the locations and sizes of DG units.

Placement of DGs units is an essential factor because improper location may lead to voltage instability and power loss. The Newton Rapson, load flow method, used in [40]. This method reduces the power loss and the cost factor very effectively, but the conventional method of load flow analysis was not applicable for distribution system because of its high $\mathrm{R} / \mathrm{X}$ ratio, a large value of resistance and reactance of the line and radial structure of the distribution system. Tuba Gozel used loss sensitivity factor for determination of the optimal size and location of DG to minimize total power loss [41]. Andrew used the Linear Programming Technique for placement of DG with multiple constraints [42]. Mallikarjuna used Simulated Annealing for determining the optimal location and size of DG units in a microgrid, given the network configuration and heat and power requirements at various load points [43]. Krueasuk used PSO to find optimal location and size of DG [44]. Lalitha used fuzzy approach to find optimal DG localization [45]. Hughifam used a multi-objective function to minimize the cost of energy losses, Investment cost of DG and Operation and maintenance cost [46]. Ochoa minimized real power loss and single phase short circuit level [47]. Celli used multi-objective approach, Genetic Algorithm (GA) has been adopted to solve the optimal placement of different types of generation simultaneously. He saved the energy in the form of greenhouse gas emission reduction [48]. Vinoth Kumar addressed minimizing the multi-objective index using a genetic algorithm for the optimal Placement of DG [49]. The proposed approach in [50] is applied to determine the optimal location and size of integrating DGs units into the distribution system.

In the optimization process of a difficult task, the method of the first choice will usually be a problem specific heuristics. These techniques using expert knowledge achieve superior performance. If problem specific technique is not applicable due to unknown system parameters (e.g., node voltage and branch currents), the multiple local minima, or nondifferentiability, Evolutionary Algorithms (EAs) have the potential to overcome these limitations [51]. EAs are a class of direct search algorithms. A conventional direct search method uses a strategy that generates variations of the design parameter vectors. Once a variation is generated, the new parameter vector is accepted or not. The new parameter vector is accepted in the case it reduces the objective function value. This method is usually 
named the greedy search. The greedy search converges fast but can be trapped by local minima. This disadvantage can be eliminated by running several vectors simultaneously which is the main idea of differential evolution (DE) algorithm.

Differential_evolution (DE) is a stochastic, population-based search strategy developed by Stern and Price [52] in 1995. DE differs significantly from other evolutionary algorithms (EA), in the sense that the direction information and the distance from the current population are used to guide the search process. In most EAs, variation from one generation to the next is achieved by applying crossover and mutation operators. If both these operators are used, the crossover is usually applied first, after which the generated offspring are mutated. The main difference between DEA and EVs in that:

- Mutation operator is applied first, then the generate trial vector is used within the crossover to generate the offspring.

- Mutation step sizes are controllable parameters and are not sampled from a prior distribution function.

In DEA, mutation step sizes are influenced by differences between individuals of the current population.

Additionally, DEA is a population-based using three operators such as crossover, mutation, and selection. It differs from another algorithm like GA is that genetic algorithms rely on crossover while DEA relies on mutation. This operation is based on the differences between randomly sampled pairs of solutions in the population.

The DEA has three advantages: finding a global minimum regardless of the initial population parameter values, converges fast, and a few control parameters are needed [53]. In DE algorithm, all solutions have an equal opportunity of being preferred as parents, and selection does not depend on their fitness values.

This dissertation proposed a DEA for finding the optimal size and location of the planned DG unit in a distribution network. The technique will minimize real and reactive power losses and improve the system overall voltage profile. The optimizations technique are performed in three stages: the network reconfiguration, the locations, and the sizes of DGs units. Different test systems are used herein to validate the effectiveness of the proposed approach. 


\section{Chapter 3}

\section{Distribution Feeder Reconfiguration For Loss Reduction}

\subsection{Introduction}

Distribution systems are generally designed and built as meshed networks, while they are operated radially. Their configurations processers may vary with manual or automatic switching operations so that all of the loads are served and increase power quality and improve system reliability and also reduce network losses. Feeder reconfiguration benefits the system by relieving the overloading of the network components by transferring loads from heavily loaded lines to partially loaded one. Network configuration is performed by changing the status of the switches. Distribution networks contain two types of switches normally closed switches (sectionalized switches) and normally open switches (tie switches). In general, feeder reconfiguration is performed when there is a significant change on loads or during contingency conditions such as sudden fault occurring on the feeder.

Additionally, to date, there is rules or standard regulation enacted to define the frequency of implementing the network reconfiguration. However, based on the literature review most distribution network application use real-time data with a discrete time step of one hour. Therefore, hourly feeder reconfiguration is used in most existing online studies to reduce distribution system losses.

In light of the literature review in chapter two, most approaches proposed so far use some heuristics, mathematical complex programming or approximate techniques and it considers taking long computation time and more memory space. Thus, the obtained results are either approximate or only local optimal solutions. Also, they require more time and memory to be computed especially for large systems. Due to this, the proposed work in this chapter is to formulate and solve the distribution feeder reconfiguration problem using a simple load flow technique for the radial distribution network. This technique involves only the implementation of a simple algebraic expression of receiving-end voltages and employing the graph theory to efficiently construct the system structure. The topology is to set the value of the substation voltage to $1 p . u$ and since the load date $(P \& Q)$ is provided and 
specified as a $P Q$ load, then branch currents become easily to obtain. The power flow results are obtained iteratively. This section is only focus on the reconfiguration technique without including distributer energy resources. The reconfiguration approach is tested on the IEEE 33 bus radial distribution system [10] has five tie-switches (dotted branches) and 32 in service branches (solid branches) with sectionalizing switches at each line and 33 nodes. The system has a total load of 3.715MW and 2.30MVAr. The base network power loss is $202.6762 \mathrm{~kW}$ and tie switches are $33,34,35,36,37$. To simplify the analysis, it assumed that the test system is presented on a per phase basis and the loads along the feeder are considered as a spot load with constant $P, Q$ loads placed at the end of the lines. It is also assumed that there is a sectionalized switch associated with each line in the system. The problem formulation considers four objectives related to minimization of the system power loss, minimization of the deviation of the nodes voltage, minimization of branch current constrain violation, minimization of feeder currents imbalance.

In this section will investigate feasible methods for solving the multi-objective problem. One possible formulation is to use weight-based objective function formulation, where different weights are assigned to each objective. The reconfiguration depends on the proper selection of weighting factors. One has to select proper values of weightings factors such that each object may be given preference as desired by the operator.

The remainder of this chapter is organized as follows. The load flow formulation is presented in Section 3.2. power system modeling using graph theory is discussed in Section 3.3. solution methodology is detailed in Section 3.4. In Section 3.5, the load flow calculation techniques are illustrated in details. The feeder reconfiguration techniques are presented in section 3.6. the fitness function is formulated in section 3.7. distribution test systems and the simulation results and discussion are presented in section 3.8 and 3.9 respectively. In section 3.10 the Test feeder performance after reconfiguration with the presence of DGs units is investigated using the IEEE 33-bus test system. The DG effect on the radial distribution system is discussed in 3.11. Conclusions are drawn in Section 3.12. 


\subsection{Load flow formulation}

To illustrate the solution methodology of the load flow analysis, let's assume that the balanced three-phase radial distribution network is represented by an equivalent circuit. Let's consider a single line diagram of a radial distribution system shown in figure 3.1. In this dissertation, the formulation of load flow is adopted with the use of graph theory. The total number of nodes beyond each feeder, branch or letteral is identified by applying graph theory topology. Known the number of nodes beyond each node would ease the computation of branch currents of each edge in the system. For example, the current of edge 1 (branch 1 between node 1 and node 2) would be the summation of all the load currents in the system, thus $B c_{1}=I_{2}+I_{3}+I_{7}+I_{4}+I_{8}+I_{5}+I_{12}+I_{10}+I_{9}+I_{6}+I_{11}$ Load currents $\left(I_{2}, I_{3}, \ldots.\right)$ can be calculated using (3.7).

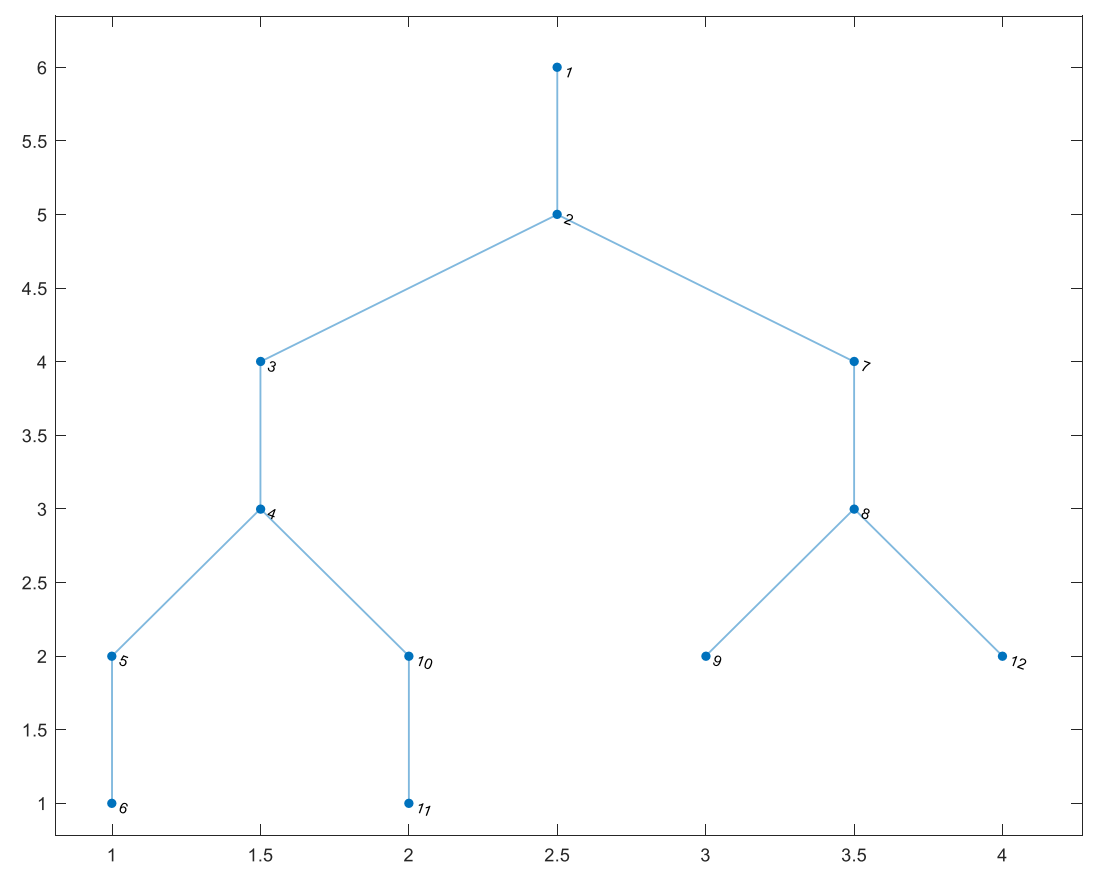

Figure 3.1 Simple line diagram of a radial distribution system 


\subsection{Power system modeling using graph theory}

This part will introduce a graph theory and describe how to use it to model a power system. Graph theory is an area of mathematics describing the study of pairwise relations between objects.

Graph theory can be applied to power systems in multiple ways. A graph is an object having two sets called vertex set and edge set [54]. The vertex set cannot be empty, but the edge set can be. The elements in the vertex set are called vertices. Each element in the edge set is a two-element subset of the vertex set. These elements are called edges.

Now, since the line data is known or provided (e.g., sending end and receiving end nodes) a power system model can be built using a graph theory idea above. Fundamentally, any buses in the power system are connected through circuit breakers and switches, therefore the circuit breakers and switches can be modeled as edges, and the buses are modeled as vertices in a graph. For example, let's consider figure 3.1, if the breaker s1 (e.g., sectionalized switch or tie switch) between the substation node (node 1) and first node next to the substation (node 2) is closed, then the switch s1 is called vertices, and the edge is created. However, if the switch s1 is open, then there is no edge between these two nods. Figure 3.2a shows a graph representation of the power system Figure 3.2b shows the same power system with s1 open. 

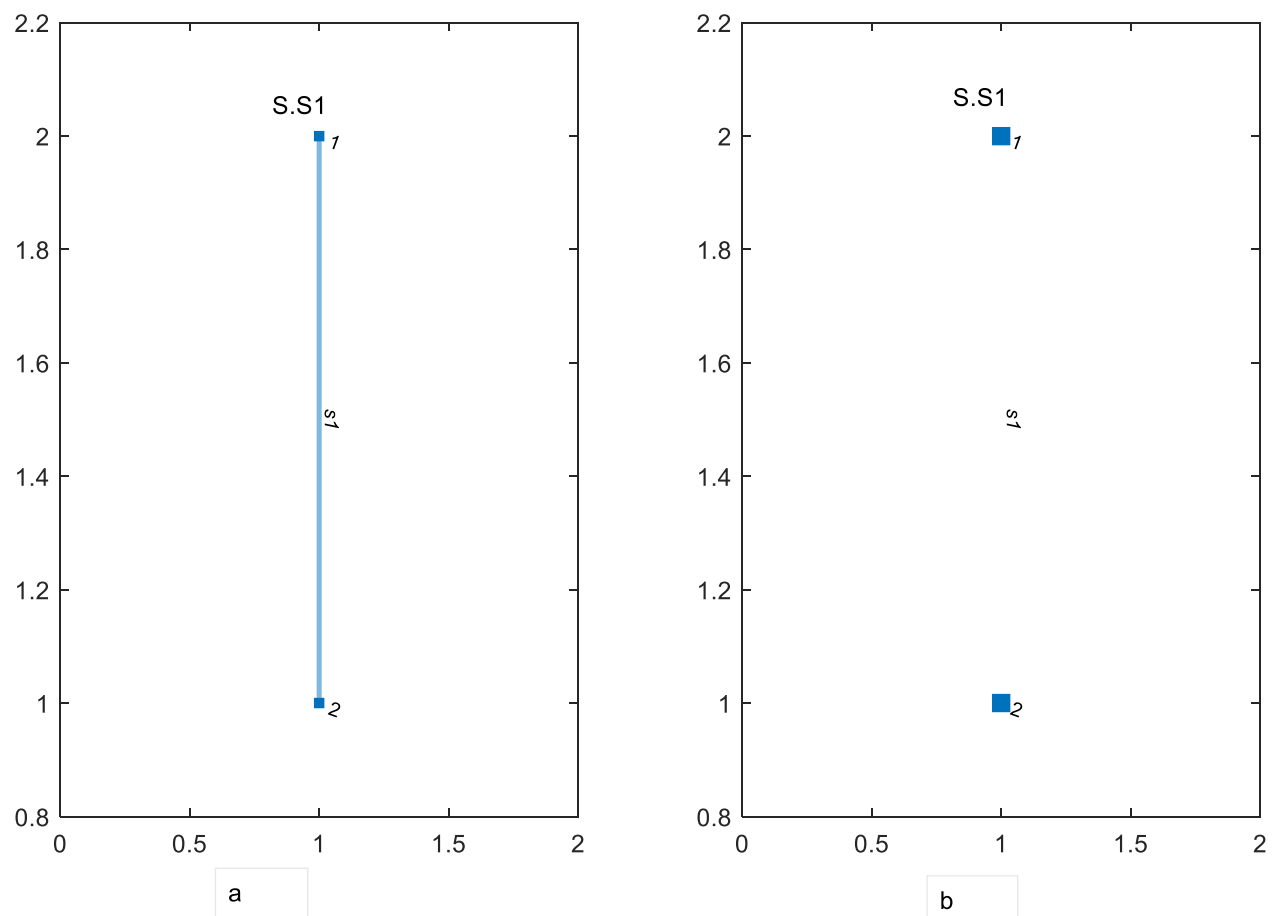

Figure 3.2 A graph representation of the power system

The results from the graph property are:

Edges: [1x1 table]: the edges are representing the branches or feeders.

Nodes: [2x0 table]: the nodes are the busses creating the branches.

If a particular node is connected with more than one node (e.g., node with connected lateral), then the Neighbors command is used to identify the connected node in a graph. For instance, let's consider figure 3.1 again node 4 has two connected nodes next to it besides node three which is the source of node 4 . Therefore, node 4 has three neighbors node 3 , node 5 , and node 10 . After specifying the neighbors of each node, the edges are created, and the network and power flow are modeled.

\subsection{Solution methodology}

The branch number, sending-end and receiving-end node of the feeder shown in figure 3.1 are given in Table 3.1. The strategy is to consider a branch at each time and then identifying the number of nodes beyond this particular branch as discussed in the previous section 
(graph theory), for instance, consider branch 1 . The voltage at the receiving-end node can be obtained as

$$
\mathrm{V}(2)=\mathrm{V}(1)-\mathrm{I}(1) \mathrm{Z}(1)
$$

Table 3-1 Solution methodology of load flow analysis

\begin{tabular}{|l|l|l|l|l|}
\hline $\begin{array}{l}\text { Branch } \\
\text { number }(j j)\end{array}$ & $\begin{array}{l}\text { Sending end } \\
m 1=I S(j j)\end{array}$ & $\begin{array}{l}\text { Receiving } \\
\text { end } \\
m 2=I R(j j)\end{array}$ & $\begin{array}{l}\text { Nodes beyond branch } \\
j j\end{array}$ & $\begin{array}{l}\text { Total } \\
\text { number of } \\
\text { Nodes } N(j j) \\
\text { beyond } \\
\text { branch } j j\end{array}$ \\
\hline 1 & 1 & 2 & $2,3,7,4,8,5,10,9,12,6,11$ & 11 \\
\hline 2 & 2 & 3 & $3,4,5,10,6,11$ & 6 \\
\hline 3 & 3 & 4 & $4,5,10,6,11$ & 5 \\
\hline 4 & 4 & 5 & 5,6 & 2 \\
\hline 5 & 5 & 6 & 6 & 1 \\
\hline 6 & 2 & 7 & $7,8,9,12$ & 4 \\
\hline 7 & 7 & 8 & $8,9,12$ & 3 \\
\hline 8 & 8 & 9 & 9 & 1 \\
\hline 9 & 4 & 10 & 10,11 & 2 \\
\hline 10 & 10 & 11 & 11 & 1 \\
\hline 11 & 8 & 12 & 12 & 1 \\
\hline
\end{tabular}

Similarly, for branch number 2

$$
V(3)=V(2)-I(2) Z(2)
$$

Since the substation voltage is known $V(1)$, if $I(1)$ is known as well (by initializing a flat voltage start for all nodes and using (3.7)), then it becomes easy to calculate the voltage of node number 2, $V(2)$ from (3.1). Once $V(2)$ is known, it is also easy to calculate $V(3)$ from (3.2). Similarly, following the same procedure all node voltages can be easily calculated. Therefore, the generalized equation is represented as follows:

$$
V(m 2)=V(m 1)-I(j j) Z(j j)
$$


where $j j$ is the branch number, it can be evaluated for $j j=1,2,3, \ldots . L N 1$ where $(L N 1=$ $N B-1): L N 1$ and $N B$ are the total number of nodes and a total number of branches respectively. the

$$
\begin{aligned}
& \mathrm{m} 1=\operatorname{IS}(\mathrm{j} j) \\
& \mathrm{m} 2=\operatorname{IR}(\mathrm{j} j)
\end{aligned}
$$

$I S(j j)$ and $I R(j j)$ are a sending-end node of branch $j j$ and receiving-end node a of branch $j j$ respectively.

The current through branch 1 is equal to the sum of the load currents of all the nodes beyond branch 1 (i.e.).

$$
\mathrm{I}(1)=\sum_{\mathrm{i}=2}^{\mathrm{LN} 1} \mathrm{IL}(\mathrm{i})
$$

Therefore, it becomes possible to calculate all the branch currents after identifying the nodes beyond all the branches. The generalized form of the current equation is:

$$
\mathrm{IL}(\mathrm{i})=\frac{\mathrm{PL}(\mathrm{i})-\mathrm{jQL}(\mathrm{i})}{\mathrm{V}^{*}(\mathrm{i})} \quad \mathrm{i}=2,3, \ldots \ldots, \mathrm{NB}
$$

where, $P L(i)$ and $Q L(i)$ is the real and reactive power loads at the $i t h$ node.

The losses of branch $j j$ are given by:

$$
\begin{aligned}
& L P(j j)=|I(j j)|^{2} R(j j) \\
& L Q(j j)=|I(j j)|^{2} X(j j)
\end{aligned}
$$

The main advantage of identifying the nodes beyond all the branches is to compute the exact current flowing through all branches, which give us the ability to quantify the current of a particular branch.

\subsection{Load-flow calculation technique}

Once all nodes beyond each branch are known, it becomes straightforward to calculate the current flowing through each branch. The load current drawn by each node is calculated using (3.7)

$$
I(j j)=\sum_{i=1}^{N(j j)} \operatorname{IL}\{I E(j j, i)\}
$$


Where $I E(j j, i)$ indicates all the nodes $i$ beyond branch $j j$

The topology herein is to initiate a constant flat voltage of all the nodes and then the branch currents are computed using (3.7). After that compute branch currents using (3.10), and then the voltage of each node is calculated using (3.3) with (3.4). The real and reactive power losses of each branch are calculated using(3.8) and (3.9), respectively. Once the new voltage values of all the nodes are computed, the convergence of the solution is then checked. The convergence is checked such that if, in successive iterations the maximum difference in voltage magnitude (DVMAX $<=0.0001$ p.u), then the solution has converged. Otherwise, if it does not converge, then the load currents are computed using the most recent voltages values, and the whole process is repeated. The load-flow algorithm for solving the radial distribution network is given in the form of a flowchart in Figure 3.2. 


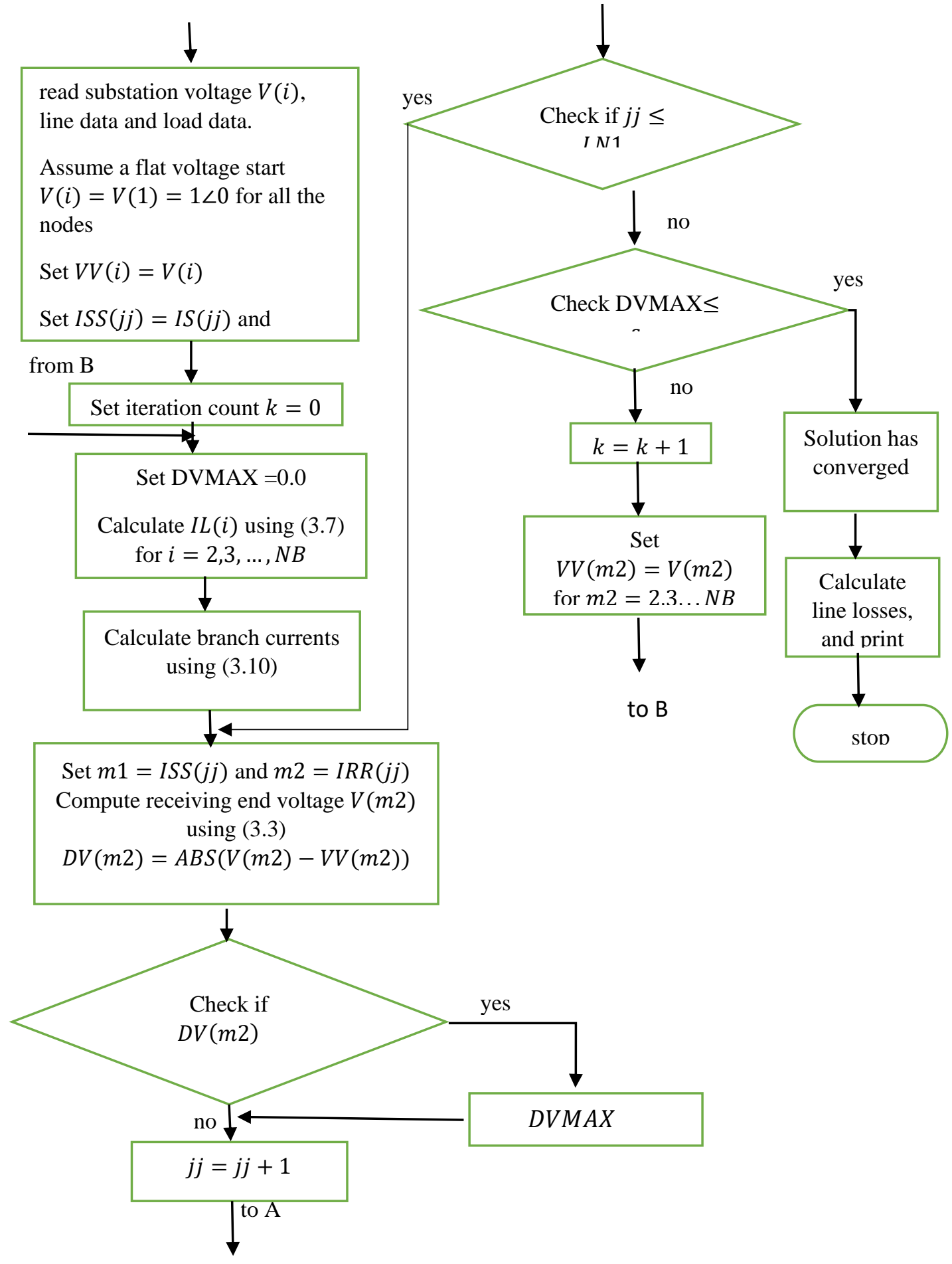

Figure 3.3 Flow-chart for load-flow calculation of radial distribution network 


\subsection{Feeder reconfiguration technique}

After the brief explanation of the power flow technique employed in this research, the reconfiguration will be discussed in details.

This research aims to investigate and solve multi-objective function, based on four objectives related to optimal real power loss minimization, deviation of the node voltages, branch current violation, and feeder currents imbalance. In fact, in reality, it is hard to have a situation in which all the objectives can be met or be in the best possible way to be satisfied simultaneously [55] [56]. Therefore, the four objectives are augmented into an objective function $\mathrm{j}$ though appropriate choice of weighting factors.

3.6.1 Real power loss $\left(X_{i}\right)$

$$
\mathrm{X}_{\mathrm{i}}=\frac{\operatorname{Ploss}(\mathrm{i})}{\operatorname{Ploss}^{0}} \quad \mathrm{i}=1,2, \ldots, \mathrm{Nk}
$$

where $\operatorname{Ploss}(i)=$ total real power loss when the ith branch in the loop is opened

$\operatorname{Ploss}^{0}=$ total power loss in the network before reconfiguration

$N k=$ total number of branches in the loop including tie branch, when the $k t h$ switch is closed.

For the best results, the value of $X_{i}$ has to be less than unity.

3.6.2 Maximum voltage deviation $\left(Y_{i}\right)$

$$
Y_{i}=\max \left|V_{s}-V_{i j}\right| \text { for } i=1,2, \ldots N k \text { and } j=1,2, \ldots, N B
$$

where $V_{s}=$ substation voltage in $p . u$,

$V_{i, j}=$ the voltage of node $j$ corresponding to the opening of the ith branch in the loop (in p.u)

$N B=$ total number of nodes in the system

The boundaries of the voltage deviation are \pm 10 , for example, if the substation voltage is equal to $1 p . u$ then the system minimum constraint is set to $V^{s} \min =0.9 p . u$ then $Y_{i}=$ $Y_{\max }=0.10$. 
The better configuration result is obtained when $Y_{i}<0.10$, if this condition satisfied then the system will have better voltage profile.

\subsubsection{Maximum branch current $\left(\mathrm{Z}_{\mathrm{i}}\right)$}

$$
\mathrm{Z}_{\mathrm{i}}=\max \left(\frac{|\mathrm{I}(\mathrm{i}, \mathrm{m})|}{\mathrm{Ic}(\mathrm{m})}\right) \text { fro } \mathrm{i}=1,2, \ldots, \mathrm{Nk} \text { and } \mathrm{m}=1,2, \ldots \mathrm{NB}-1
$$

where

$|I(i, m)|$ is the current magnitude of branch $m$ when the ith branch in the loop is opened. $I c(m)$ is the maximum current carrying capacity of branch $m$.

Although the branch currents of the system are set to be less than or equal to the rated capacity, the $15 \%$ overloading is allowed [5] for each branch. Meanwhile, during the iterative process, if any branch is overloaded more than $15 \%$ or in another word, if $Z_{i}>$ 1.15 , then the objective function value is set to very high value.

\subsubsection{Feeder load balancing $\left(\mathrm{U}_{\mathrm{i}}\right)$}

$$
U_{i}=\max \left(\frac{I F F_{i}^{\max }-I F_{i, j}}{I F F_{i}^{\max }}\right) \text { for } 1=1,2, \ldots, N K \text { and } j=1,2, \ldots N F
$$

where

$N F$ is the total number of feeders,

$I F_{i, j}$ is the current of feeder $j$ corresponding to the opening of the $i t h$ branch in the loop

$I F_{i}^{\max }$ is the maximum of all the feeder currents corresponding to the opening of the ith branch in the loop, that is $I F_{i}^{\max }=\max \left(I F_{i, j}\right)$

From (3.14) it is obvious that a better feeder load balancing can be achieved if the value of $U_{i}$ is low. In this case, a limit [5] is imposed on $U_{i}$ such that $U_{i}=U_{\max }=0.25$ this indicates that the maximum deviation of feeder current will be $25 \%$ with respect to the maximum value of the feeder current.

\subsection{Fitness function formulation}

The four objectives described by $(3.11),(3,12),(3.13)$, and (3.14) are combined through appropriate weighting factors to form the objective function of the radial distribution system as follows: 


$$
\operatorname{Min} j_{i}=w_{1} X_{i}+w_{2} Y_{i}+w_{3} Z_{i}+w_{4} U_{i} \text { for } i=1,2, \ldots, N k
$$

The reconfiguration depends on the proper selection of the weighting factors $\left(\mathrm{w}_{1}, \mathrm{w}_{2}, \mathrm{w}_{3}, \mathrm{w}_{4}\right)$. One has to select proper values of weighing factors such that each object may be given preference as desired by the operator. In this research three cases for different weighting factors are considered here.

The strategy of the reconfiguration herein is that at the beginning all the tie switches are considered. In other words, first, run the load flow program under normal condition (initial case) to compute the voltage difference across all the tie switches. Detect the open ties switch which has the maximum voltage difference, then consider closing this tie switch first, as it is expected that this switch will cause a maximum loss reduction, minimum nodes voltage deviation, minimum branch current constraint violation, and better load balancing. Closing tie switch and opping another sectionalized switch will result transferring lodes between feeders. The same strategy is repeated for the remaining tie switches in the next iterations.

The topology of the switch operation is that when closing a particular tie switch the loop is formulated, and the number of nodes and branches includes the tie switch is observed, and then the opening of each branch in the loop is an option (the radial structure is retained). A number of the loop (mesh) is equal to the number of ties. The objective function is evaluated for each option, and the optimal solution is computed. For the illustration purpose, let's consider closing a tie $\boldsymbol{x}$, and the loop is formulated, the observed total number of branches are ten including tie branch, then open and close each branch at a time which means evaluate the objective function (3.15) ten times such that, $j_{x}=\left(j_{1}, j_{2}, j_{3}, \ldots \ldots . j_{10}\right)$. the optimal solution will be

$$
\mathrm{OS}=\min \left\{\mathrm{j}_{1}, \mathrm{j}_{2}, \mathrm{j}_{3}, \ldots \ldots \mathrm{j}_{10}\right\}
$$

\subsection{Distribution test systems}

To validate the performance of the presented approach two test systems are considered. The 11-kV rural radial distribution system is having two substations, four feeders, 70 nodes, and 79 branches including tie branches as shown in Figure 3.3. This network has 11 tie switches, and these tie switches are open under normal operating condition. Data 
for this system can be found in [5]. The second test feeder considered herein is the 33-bus IEEE radial distribution test system [10].

\subsection{Simulation Results and Discussion}

\subsubsection{The 11-kV rural radial distribution system}

The reconfiguration algorithm is illustrated and tested on an $11 \mathrm{kV}$ radial distribution system having two substations, four feeders, 70 nodes, and 79 branches including tie branches as shown in Fig.3. This network has 11 tie switches, and these tie switches are open under normal operating condition. Data for this system can be found in [5]. Results before reconfiguration and after reconfiguration are given in table 3.2.

Solution algorithm

Step-: Read the system data.

Step-2: Run the load flow program.

Step-3: Compute the voltage difference across the open tie switches $\Delta V_{t i e}(i)$.

Step-4: Identify the open tie switch across which the voltage difference is maximum and its code $k$ such that, $\Delta V_{\text {tie, } \max }=\Delta V_{\text {tie }}(k)$.

Step-5: Select the tie switch ' $k$ ' and identify the total number of loop branches $N_{k}$ including the tie branch when the tie switch $k$ is closed.

Step-6: Opening one branch at a time in the loop and run the load flow program and evaluate the value for each objective, compute $X_{i}, Y_{i}, Z_{i}$, and $U_{i}$ using (3.11) to (3.14) respectively, and then compute $j_{i}$ using eqn. (3.15).

Step-7: Obtain the optimal solution for the operation of the tie switch $k . O S_{k}=\min \left\{j_{i}\right\}$, and open the branch corresponding to $\min \left\{j_{i}\right\}$. Noting that if the value of $O S_{k}$ and $O S_{k-1}$ are the same then the same switch is opened again and this tie switch is not carried out.

Step-8: Check $N_{\text {tie }}=N_{\text {tie }}-1$.

Step-9: Check if $N_{t i e}=0$. If yes, go to step 11 otherwise go to next step.

Step-10: Rearrange the coding of the rest of the tie switches, and go to step-2.

Step-11: Print the output results and stop. 


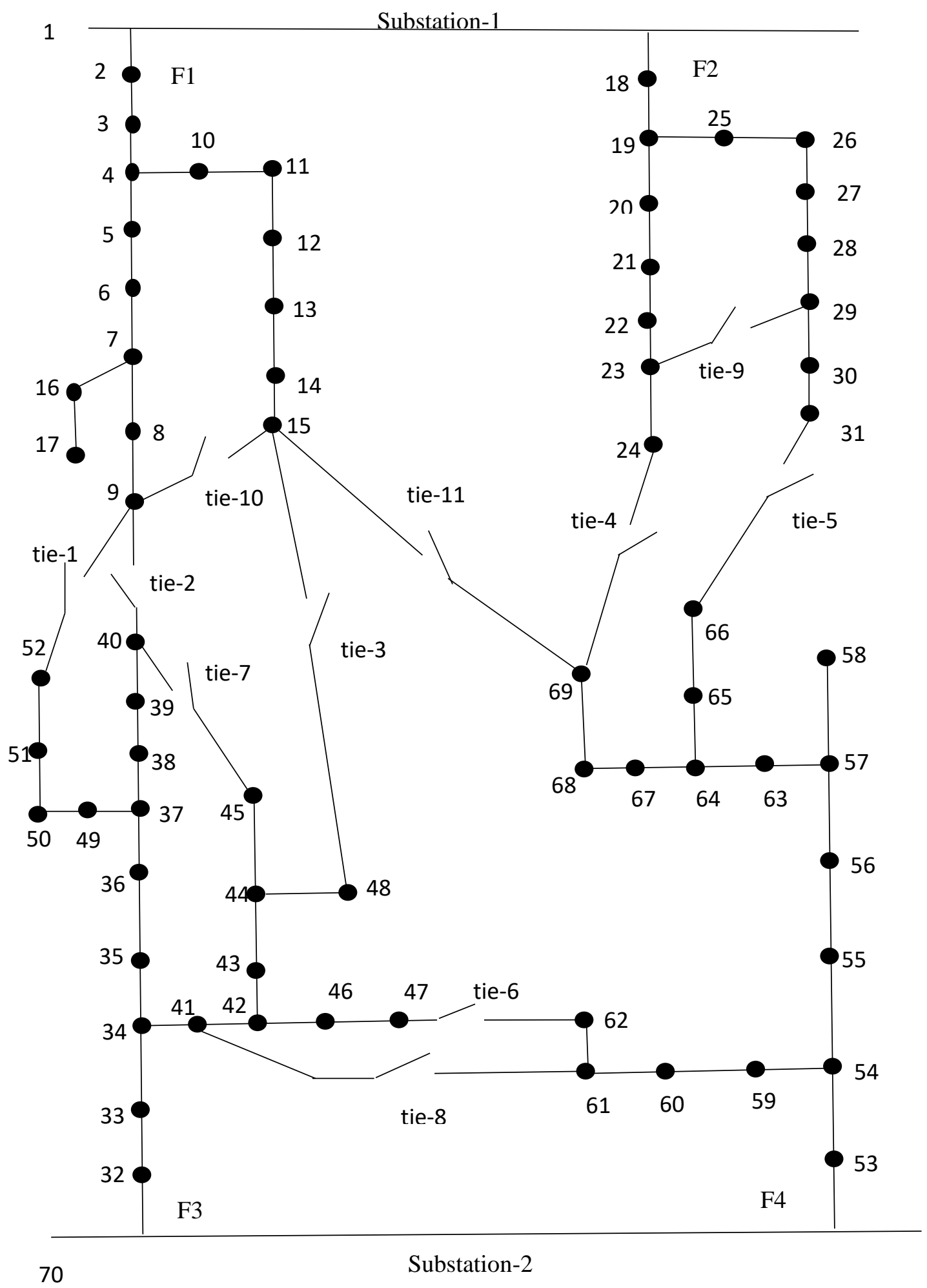

Figure 3.4 Distribution system with two substations and 11 ties switches before reconfiguration. 
Results of load flow before reconfiguration are given in table 3.2. Table 3.3 shows the results of different cases after reconfiguration. The real power loss before reconfiguration as shown in table 3.2 is $229.0891 \mathrm{~kW}$ (3-phas), and the minimum voltage $V_{\min }=$ 0.9046 p.u occurred at node number 69. In the first case of table 3.3 , all the four objectives are considered. The reconfiguration results show a promised result, it can be seen that the real power loss is reduced to $210.9385 \mathrm{~kW}$. This means the reduction of power losses is $18.1505 \mathrm{~kW}$, meanwhile, the minimum system voltage is $V_{\min }=$ 0.9269 p.u occurred at node 51 . The system minimum voltage has improved from 0.9046 p.u to 0.9269 p.u. in addition, the results after reconfiguration show that the feeder currents are more balanced as compared to that computed before reconfiguration. In the first case, only four tie switch operation was required to get the optimum results. The procedure is that in the first iteration the maximum voltage difference occurred at tie switch number four, therefore consider closing this tie switch first. Next, the maximum voltage difference occurred at tie number six and then tie nine and tie eight, but for the case of tie eight operation closing and opening branches did not make any difference, and the results remained the same. So there is no improvement of the optimal objective function value for this tie switch operation. Next, consider closing tie one. In this case, there is some improvement in the value of the optimal solution.

For case two, only power loss reduction index is considered in the objective function. Thus, $w_{1}=1$ and $w_{2}=w_{3}=w_{4}=0$. From table 3.3 it can be seen that the power loss is $202.42 \mathrm{~kW}$. This means the system power loss has improved by $26.6691 \mathrm{~kW}$. in this case the system minimum voltage occurred at node 31 , but however, the feeder currents are not that balanced as compared to case one. In this case, five tie switch operation was required. In case three, consider only two objectives (power loss reduction and maximum voltage deviation). From table 3.3 it can be observed that the real power loss is reduced by $22.64 \mathrm{~kW}$, and the minimum system voltage occurred at node 31 . The feeder currents are not that balanced as compared to that in case one. There is a slightly change in the currents, because in this case, only four tie operation were required to get the optimal solution. The final configuration is given in Figure 3.4. 
Table 3-2 load flow results before feeder reconfiguration

\begin{tabular}{|c|l|l|}
\hline $\begin{array}{l}\text { Real power loss } \\
(\mathrm{kW})\end{array}$ & $\begin{array}{l}\text { Minimum system voltage } \\
V_{\min }(p . u)\end{array}$ & Feeder currents $(A)$ \\
\hline \multirow{2}{*}{229.0891} & $V_{\min }=V_{69}=0.9046$ & $I F_{1-2}=I F_{1}=102.3402$ \\
\cline { 3 - 3 } & & $I F_{1-18}=I F_{2}=110.5245$ \\
\cline { 3 - 3 } & & $I F_{70-32}=I F_{3}=160.2338$ \\
\cline { 3 - 3 } & $I F_{70-53}=I F_{4}=149.6140$ \\
\hline
\end{tabular}

Table 3-3 load flow results after feeder reconfiguration

\begin{tabular}{|c|c|c|c|c|}
\hline $\begin{array}{l}Z_{2} \\
0 \\
0\end{array}$ & $\begin{array}{c}\text { Power losses } \\
k W\end{array}$ & $V_{\min }(p . u)$ & $\begin{array}{l}\text { Weighting } \\
\text { factors }\end{array}$ & Feeder currents $(A)$ \\
\hline \multirow{4}{*}{1} & \multirow{4}{*}{210.9385} & \multirow{4}{*}{$\begin{array}{l}V_{\min }= \\
V_{51}=0.9269\end{array}$} & $w_{1}=2$ & $I F_{1}=132.1800$ \\
\hline & & & $w_{2}=10$ & $I F_{2}=133.0157$ \\
\hline & & & $w_{3}=1$ & $I F_{3}=130.5629$ \\
\hline & & & $w_{4}=1$ & $I F_{4}=125.0315$ \\
\hline \multirow{4}{*}{2} & \multirow{4}{*}{202.4217} & \multirow{4}{*}{$\begin{array}{l}V_{\min }= \\
V_{31}=0.9302\end{array}$} & $w_{1}=1$ & $I F_{1}=113.1240$ \\
\hline & & & $w_{2}=0$ & $I F_{2}=133.0157$ \\
\hline & & & $w_{3}=0$ & $I F_{3}=136.4268$ \\
\hline & & & $w_{4}=0$ & $I F_{4}=135.1105$ \\
\hline \multirow{4}{*}{3} & \multirow{4}{*}{204.7918} & \multirow{4}{*}{$\begin{array}{l}V_{\text {min }}= \\
V_{31}=0.9341\end{array}$} & $w_{1}=2$ & $I F_{1}=113.1240$ \\
\hline & & & $w_{2}=10$ & $I F_{2}=133.1042$ \\
\hline & & & $w_{3}=0$ & $I F_{3}=136.4268$ \\
\hline & & & $w_{4}=0$ & $I F_{4}=135.1105$ \\
\hline
\end{tabular}




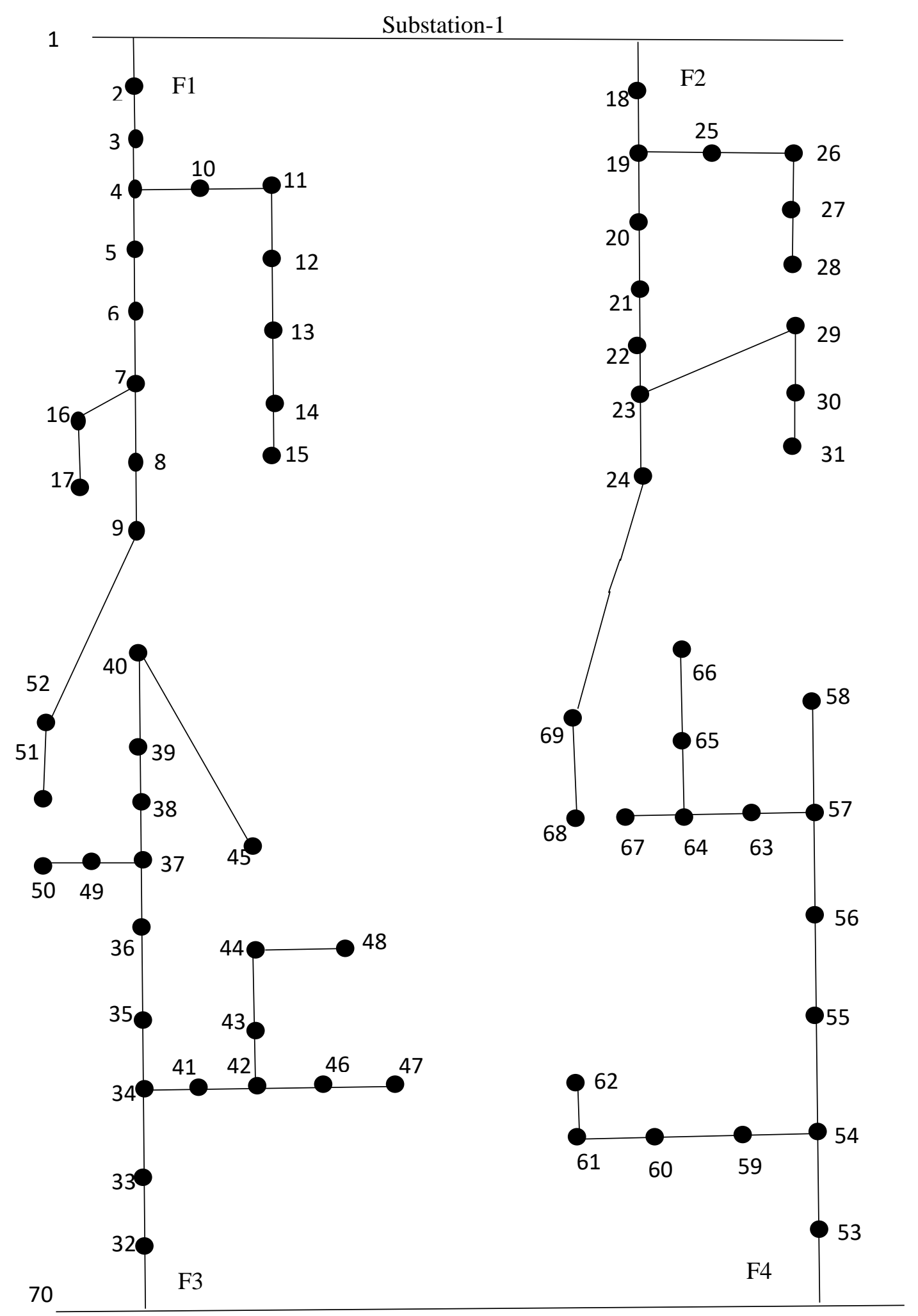

Substation-2

Figure 3.5 Distribution system performance after reconfiguration 


\subsubsection{IEEE 33-bus radial distribution system}

The assumption herein is that the test system is presented in a per phase basis and the loads along the feeder are considered as a spot load with constant P, Q loads placed at the end of the lines. Also, it is assumed that each load is associated with the sectionalized switch.

Figure 3.6 represents the IEEE 33 bus radial distribution system [10] has five tie-switches (dotted branches) and 32 in service branches (solid lines) with sectionalizing switches at each line and 33 nodes. The system has a total load of 3.715 MW and 2. 30 MVAr. The base network power loss is $202.6762 \mathrm{~kW}$ and tie switches are $33,34,35,36,37$.

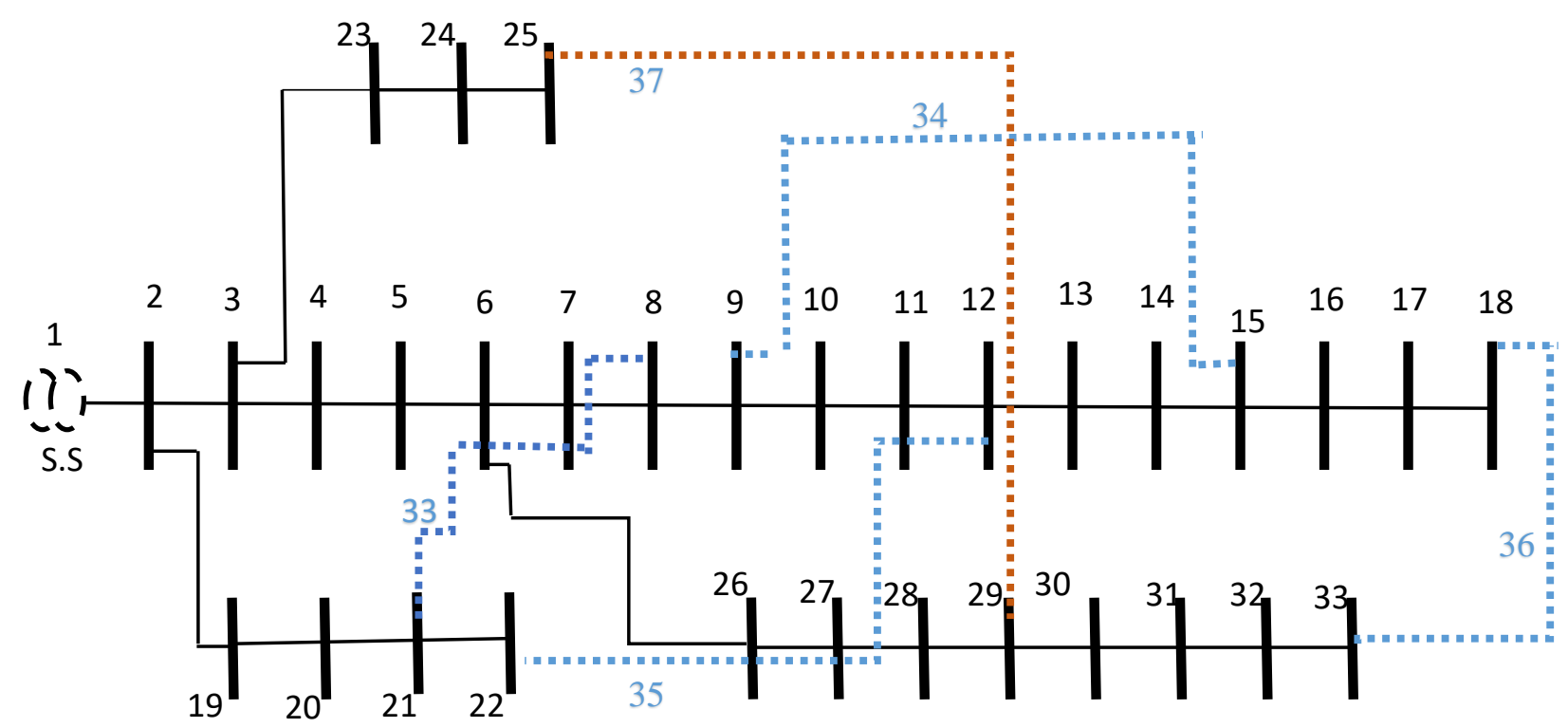

Figure 3.6 Single line diagram of IEEE 33 bus distribution system

The reconfiguration technique of this test system is implemented by following the same strategy above. This test system has five ties in which the number of loops will be five and five cases will also be conducted figure 3.7. 


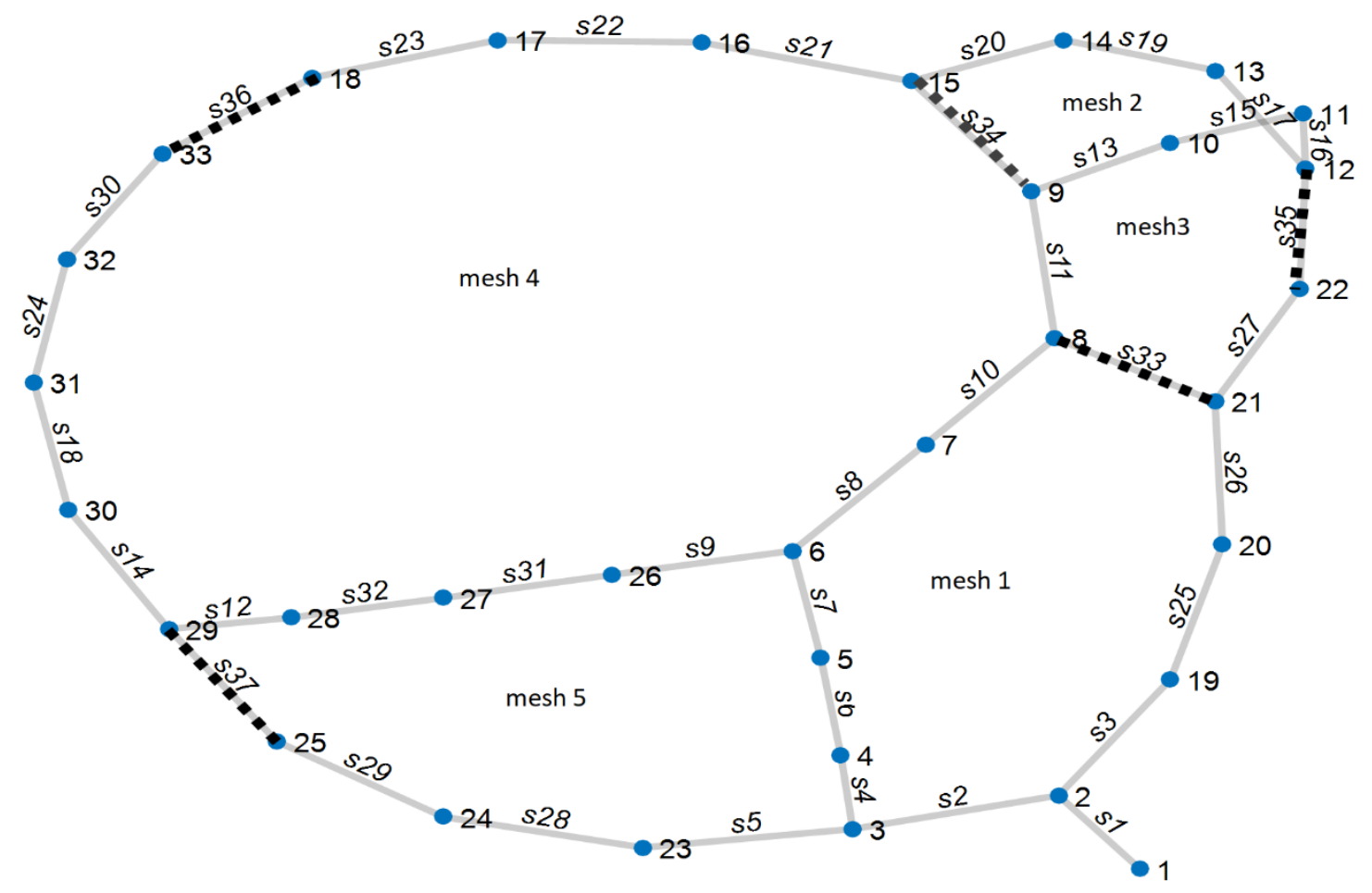

Figure 3.7 loop formulation of the 33-bus test system

Different case scenarios are considered. The simulation results of load flow before and after reconfiguration are shown in Table 3.4. For the first case study, the maximum voltage difference occurred at tie 3 which is between node 22 and node 12. It's seen that the optimal solution or optimal power flow pattern happened when closing tie 3 and opening section switch between node 8 and 9 . results of tie 3 operations. The real power loss is $140.4143 \mathrm{~kW}$ this means a reduction of real power loss is $36.9501 \mathrm{~kW}$. Minimum system voltage has improved from 0.9198 p.u to 0.9327 p.u, and that after reconfiguration it occurred at node 33 instead of node 18. Figure 3.8 shows the radial configuration of the network after the first switching operation. For the second case, the maximum voltage difference occurred at tie 5 which is between node 25 and node 29 . The results of this case show that the real power loss is dropped to 139.3385 which mean the reduction is $1.0758 \mathrm{~kW}$. Also, the minimum system voltage has improved to 0.9379 p.u. Figure 3.9 shows the optimal radial configuration of the network after the second switching operation. The third case, the power flow results that the maximum voltage difference occurred at tie 4 which is between node 18 and 33 branch number 36 (see figure 3.6). The results of case 4 and 5 also show 
no improvement on the power loss reduction and voltage profile. Therefore, these tie switches will remain open.

Table 3-4 feeder reconfiguration results of the 33-bus system

\begin{tabular}{|l|l|l|}
\hline Real power loss $(k W)$ & $\begin{array}{l}\text { Minimum system } \\
\text { voltage } V_{\min }(p . u)\end{array}$ & Feeder current $(A)$ \\
\hline 177.3644 & $V_{\text {min }}=V_{18}=0.9198$ & 345.1304 \\
\hline Case 1 140.4143 & $V_{\text {min }}=V_{33}=0.9327$ & 345.1304 \\
\hline Case 2 139.3385 & $V_{\text {min }}=V_{33}=0.9379$ & 345.1304 \\
\hline
\end{tabular}

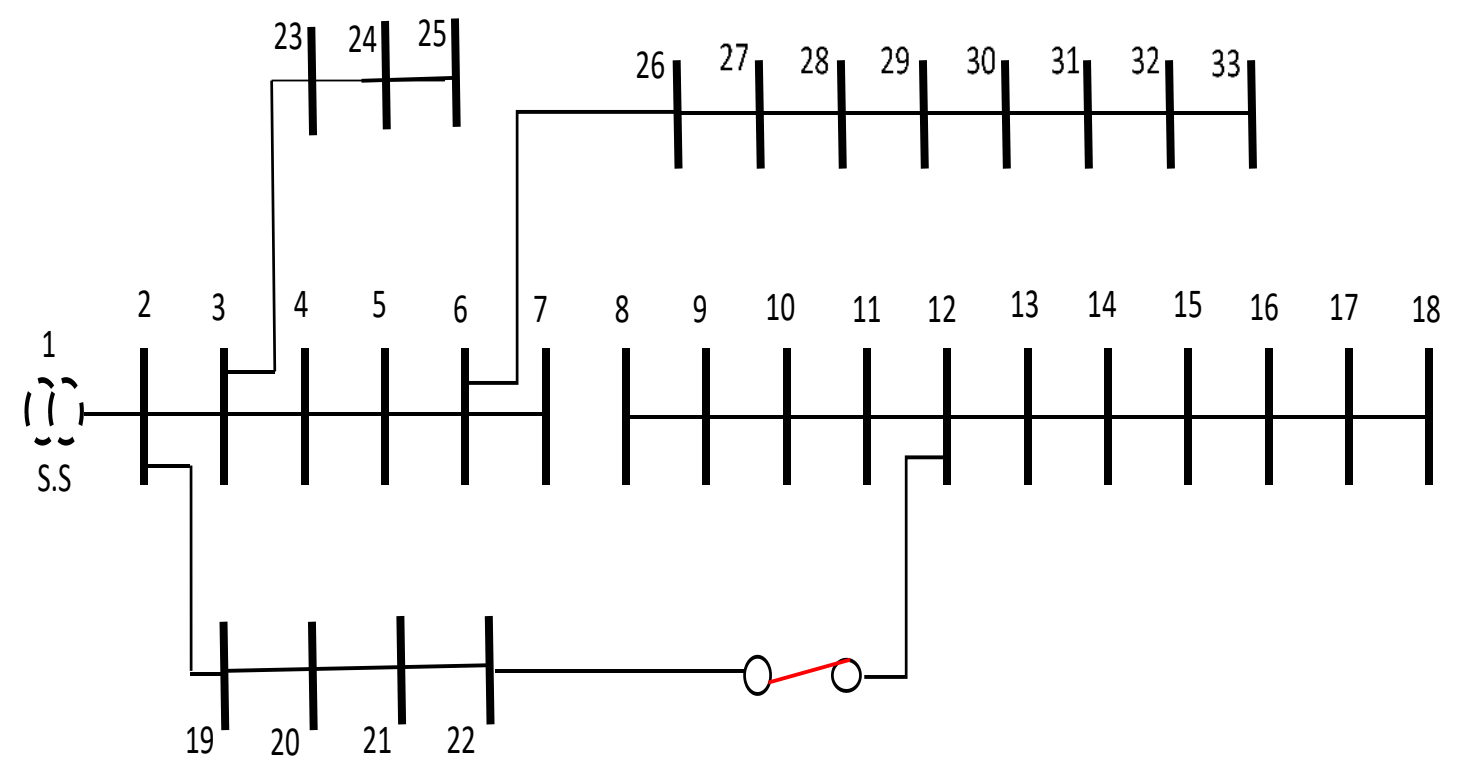

Figure 3.8 Distribution system with tie-3 closed (case 1) 


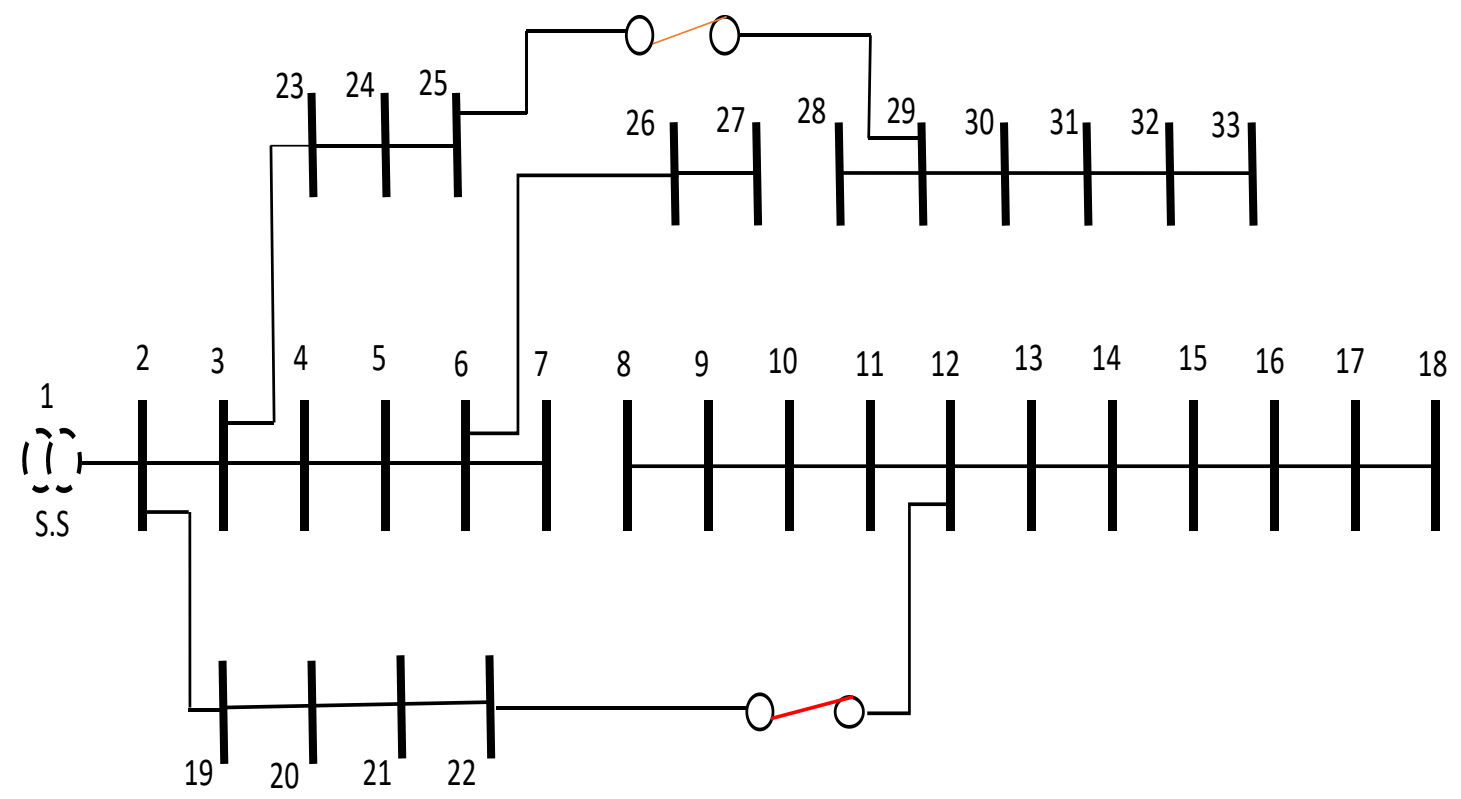

Figure 3.9 Distribution system after reconfiguration case 2 (tie-5 operation)

\subsection{Test feeder performance after reconfiguration with the presence of DGs units}

Distributed generation is operated according to its role in the system. Two main modes of DG connection can be distinguished. Operating as a backup source within a microgrid, operating in parallel with the distribution system. The operation of DG in parallel with the distribution system can contribute reducing losses, and voltage drop as well as relieves overburdened transmission and distribution facilities [57]. Distributed generation helps to reduce losses as it locally generates power demanded by loads, rather than producing it in large generation centers and forcing it to travel long distances to consumption points. The reduction achieved will depend on the generator's rated power and location. The characteristic of the IEEE 33-bus test feeder after reconfiguration is investigated under the presence of existing DGs units. The generation units by independent producers can be randomly located and provided they fulfill the connection rules, the main results of the study can be useful for the utility, which could decide whether the ratings and locations of the generation units are adequate or not. 
Distribution system power losses depend on network topology and the amount, location and output power of the integrated DGs units. The integration of DGs units adds a positive active power injection to the grid (injects negative current). In this part, the installation node and capacity of DGs are not optimum. The reason is that owners of DGs determine the installation location and capacity of the units to improve their economic benefits. Additionally, the owners of the DGs are usually individuals and non-utilities. Therefore, it becomes noncontrollable, and in this case, the size and location cannot be demonstrated. If the owner of DGs is the utility company, then the choice of its locations and sizing is important because additional DGs may cause an increase in power losses, this phenomena brings the need for optimization techniques and opens a vast research area for the interested researcher. This optimization permits the best location of generators to be found so that the power losses in the distribution systems are minimized. The determination of finding the optimum locations are changed as the load variations.

Table 3.5 shows the simulation results of replacing the DGs unit at different buses with different sizing (single phase network).

Table 3-5 Different case studies of the DGs unit replacement

\begin{tabular}{|l|l|l|l|}
\hline location & $\begin{array}{l}\text { DG Size }(\mathrm{kW}) \text { "real } \\
\text { output power." }\end{array}$ & $\begin{array}{l}\text { System Real Power } \\
\text { Loss }(\mathrm{kW})\end{array}$ & $\begin{array}{l}\text { Current from substation } \\
(\mathrm{A})\end{array}$ \\
\hline Base case & - & 139 & 345.1304 \\
\hline 30 & 300 & 93.4376 & 301.2559 \\
\hline 30 & 400 & 86.7577 & 283.2619 \\
\hline 16 & 300 & 124.9642 & 301.2559 \\
\hline 16 & 400 & 138.6488 & 283.2619 \\
\hline 6,24 & 200,100 & 111.7453 & 301.2057 \\
\hline 18,33 & 220,350 & 117.4423 & 254.6760 \\
\hline
\end{tabular}

The study provided here is aimed at analyzing the impact of DG units on the system performance. From the presented results in table 3.5, some conclusions can be derived. For instance, there is a significant improvement in the system real power losses. Also, the provided current from the sub-station is smaller. This apparently due to the provided power from the connected DGs units. It is evident that, despite that randomness, the contribution 
of the DGs units remains helpful even with multiple connected units and different sizes. Additionally, taking into account the distribution system characteristic when integrating the DGs units. In other words, the feeder hosting capacity has to be considered.

\subsection{DG effect on the radial distribution system}

High penetration of distributed generation (DG) such as photovoltaic (PV) and wind turbines (WT), have caused new challenges such as voltage rise and reversed power flows. This continually growing use of DG in medium-voltage distribution networks will profoundly impact the development of future electrical systems.

The grids were designed to transfer power from generating units connected to the high voltage grids towards the end consumers connected to the low voltage grids. With changed power mix, power flows in the system will change resulting in possible grid problems. One of the main problems is keeping the voltage within the operational limits of the system (0.95 1.05 p.u). When the generation exceeds the consumption in a distribution network, the power will flow from the low voltage network towards the high voltage network (reverse power flow) which will cause the voltage to rise in the low voltage network. Reactive power support from DG units can be a valuable resource to mitigate the problem. Reactive power is necessary to operate the power system. The primary source of reactive power is synchronous generators. If this source is shut down, the reactive power must come from another source. DGs units can provide reactive power by using inverters. Taking into an account using reactive power control for supporting voltage control, minimize system losses, reducing congestion, and compensating the consumer's reactive power demand.

Distribution networks are normally built up in the following way: The high voltage transmission network is connected to the medium voltage distribution network via a primary substation. A number of feeders are connected to this substation. These feeders are in general radially connected. Underground cables are mostly used in these feeders, and they have capacitive characteristics, so they produce reactive power. Loads connected to these feeders are mostly resistive and have a power factor very close to 1 . Some big loads are connected to the medium voltage network, and they are required to have a power factor 
within a range of 0.9 inductive and 0.9 capacitive [58]. The voltage drop along the feeder can be approximated by [59]:

$$
\Delta \mathrm{V} \approx \frac{\mathrm{R} * \mathrm{P}_{\text {load }}+\mathrm{X} * \mathrm{Q}_{\text {load }}}{\mathrm{V}_{\mathrm{n}}}
$$

where,

$\Delta V \quad$ Voltage change across the line

$P_{\text {load }}$ Active power consumption by the load (negative)

$R \quad$ Resistance of the line

$Q_{\text {load }}$ Reactive power consumption by the load (negative)

$X \quad$ Reactance of the line

$V_{n} \quad$ Nominal voltage

The voltage in distribution networks with no DG decreases therefore from the primary substation to the end of the feeder. When DGs are connected, the power flow can be reversed. So the voltage can be higher at the end of the feeder than at the primary substation thus (3.17) becomes:

$$
\Delta \mathrm{V} \approx \frac{\mathrm{R} *\left(\mathrm{P}_{\text {load }}+\mathrm{P}_{\mathrm{DG}}\right)+\mathrm{X} *\left(\mathrm{Q}_{\text {load }}+\mathrm{Q}_{\mathrm{DG}}\right)}{\mathrm{V}_{\mathrm{n}}}
$$

If the generation is a lot higher than the demand, the voltage rise can exceed the limits. To avoid this, the reactive power in the generation units can be utilized. Typically, loads have a power factor close to unity. DGs units (e.g., PVs, WTs) are usually connected through an inverter that can adjust the active and reactive power almost free but limited by the current limit of the inverter. When a DG unit is operated inductively, it consumes reactive power so $Q_{D G}$ comes negative which lowers the voltage. Capacitive operation however injects reactive power which increases the voltage. The impact that reactive power adjustments have depends highly on the R/X ratio of the lines. In medum voltage grids, the $\mathrm{R} / \mathrm{X}$ ratio is usually around 1 , so active and reactive power have equal an impact on the voltage rise. Thus, DGs units can generate or consume reactive power Q. this impact is invistigated unsing the IEEE 33-bus test system presented above. Table 3.6 show the results of positive and negative output reactive power of the DG inverter. 
Table 3-6 The impact of the positive and negative output Q of the DG inverter

\begin{tabular}{|c|c|c|c|c|}
\hline \multirow{2}{*}{ location } & \multicolumn{2}{|l|}{ DG Size } & \multirow{2}{*}{$\begin{array}{l}\text { System Real } \\
\text { Power Loss }(k \mathrm{~W})\end{array}$} & \multirow{2}{*}{$\begin{array}{ll}\text { Current } & \text { from } \\
\text { substation }(\mathrm{A}) & \end{array}$} \\
\hline & P_outout & Q_output & & \\
\hline \multirow{2}{*}{30} & \multirow{2}{*}{300} & 200 & 84.3880 & 266.1626 \\
\hline & & -200 & 119.7280 & 339.3522 \\
\hline \multirow{2}{*}{30} & \multirow{2}{*}{400} & 100 & 84.3880 & 266.1626 \\
\hline & & -100 & 93.4376 & 301.2559 \\
\hline \multirow{2}{*}{16,22} & \multirow{2}{*}{300,100} & 100,50 & 146.7896 & 257.9406 \\
\hline & & $-100,-50$ & 119.1757 & 310.5145 \\
\hline \multirow{2}{*}{16} & \multirow{2}{*}{400} & 150 & 176.4969 & 258.0045 \\
\hline & & -150 & 121.5862 & 310.5401 \\
\hline \multirow{2}{*}{6,24} & \multirow{2}{*}{200,100} & 100,75 & 101.0301 & 270.2650 \\
\hline & & $-100,-75$ & 127.0945 & 334.4468 \\
\hline \multirow{2}{*}{18,33} & \multirow{2}{*}{220,350} & 120,200 & 125.9726 & 211.8366 \\
\hline & & $-120,-200$ & 123.9099 & 310.4632 \\
\hline
\end{tabular}

\subsection{Conclusion}

In this chapter, a new approach has been proposed to reconfigure the radial distribution networks with the impact of the existed DG units. Feeder reconfiguration is performed by changing the open/close status of switches. Primary distribution networks contain two types of switches, known as tie switches (normally open) and sectionalizing switches (normally closed). These switches are designed for both protection and configuration purposes. A whole feeder or part of a feeder may be served from another feeder by closing a tie switch linking the two while an appropriate sectionalizing switch must be opened to maintain the radial structure of the system. In this research, the problem is formulated as a multi-objective problem dealing with four objectives related to minimization of the system 
power loss, minimization of the deviations of the nodes voltage, minimization of branch current violation and minimization of feeder's currents imbalance. Different loss reduction and load balancing scenarios have been investigated. For instance, simulation results study before and after network reconfiguration, with and without including DGs, and after reconfiguration with the presence of single and multiple DG units. The proposed methods are tested on 70-node $11 \mathrm{kV}$ and 33-bus systems.

The increase of DER connected to the distribution network results in possible grid problems. The main problem is the reactive power support which is the subject of the last part of this chapter. The primary source of reactive power support comes from conventional power plants and with an increasing share of DER. DGs units can provide or consume reactive power. This advantages of the DER can decrease the use of the capacitor bank equipment. In other words, DER can be coordinated with a voltage regulator and to contribute to regulating the system node voltages by adjusting its output power. 


\section{Chapter 4}

\section{Distribution Feeder Reconfiguration for Power Loss Reduction Using Fast Genetic Algorithm (FGA) Technique}

\subsection{Introduction}

Genetic algorithms are global optimization algorithms based on the mechanics of natural selection and genetics. They employ a structured yet randomized, parallel multipoint search strategy that is biased toward reinforcing search point of "high fitness," i.e., points at which the function being minimized has relatively low values. Genetic algorithms are similar to simulated annealing [60] in that they employ random ( probabilistic) search strategies. However, one of the obvious distinguishing features of genetic algorithms is their effective implementation of parallel multipoint search. According to the research work conducted in the past and the demand of having an easy and straightforward approach dealing with the optimization problems of distribution feeder reconfiguration, this chapter is tending to improve the genetic algorithm processes. In this chapter, the fast genetic algorithm FGA is developed. The novelty of this approach is that, in contrast to the traditional GA and all other approaches using binary parameters and aiming in finding the optimal global solution of any complex problem. We do not use the binary representation of the fitness function parameters instead integer parameters have been used, and also we construct the population matrix in a different way. Basically, the initial population is performed by closing the tie switches with the maximum voltage difference in order to formulate mesh networks and then opening sectionalized switches one by one for each mesh to maintain the radial structure of the system. As far as the computation time is considered, the Tabu lists with heuristic rules are also employed in the searching process to enhance performance.

Most distribution systems configured radially. There are numerous numbers of different types of switches in the distribution networks, the focus of this research would be on the normally open tie switches and normally closed sectionalized switches. This large number of the switch in the grid would tend to a tremendous number of switching operations. Therefore, network reconfiguration has become a complex and challenging decisionmaking process for system operators. 
Although there has been much work done on modifications and improvements to the genetic algorithms, this chapter will present FGA to solve the multi-objective problem optimally. The proposed method herein defers from the others in such a way the population matrix is structured differently, the network is converted to a meshed pattern and becomes easy to study and solve, and the conversion speed is improved. Most reconfiguration problems are formulated as a mixed integer nonlinear optimization problems. The proposed

FGA is dealing with an integer parameter type because the parameters herein is a switch status ( e.g., switch numbers). Integer programming with FGA involves several modifications of the traditional algorithm. The new approach will be tested and validated on revised IEEE 16-bus test system.

The remainder of this chapter is arranged as follows. Section 4.2 presents the problem formulation. Section 4.3 provides the encoding and initialization techniques of the proposed FGA. In Section 4.4 presents the initialization techniques of the FGA. Crossover and mutations are described in sections 4.5 and 4.6, respectively. In section 4.7 the fitness function is formulated based on the objectives and operational constraints. Section 4.8 draws the flow-chart of the proposed FGA. The simulation results are presented in Section 4.9. Finally, conclusions are drawn in Section 4.10.

\subsection{Problem formulation}

In the open loop radial distribution system, each radial feeder is divided into load sections with sectionalizing switches (normally closed and has connections to other feeders via a tie switches ( normally open). The loss minimization reconfiguration problem of the distribution system is to decide the position of the sectionalizing switches (open/close). This part of the dissertation focuses on the minimization of the real power loss and the number of switching subjected to the radial network structure.

Figure 4.1 shows a sample distribution system contains three feeders with thirteen normally closed sectionalizing switches and three normally opened tie switches, namely s15, s21, and s26. For instance, transferring load 11 from feeder 2 to feeder 1 will require closing switch s15 and opening switch s19. Closing a switch should always follow the opening of another switch (to maintain the radial structure of the system). 


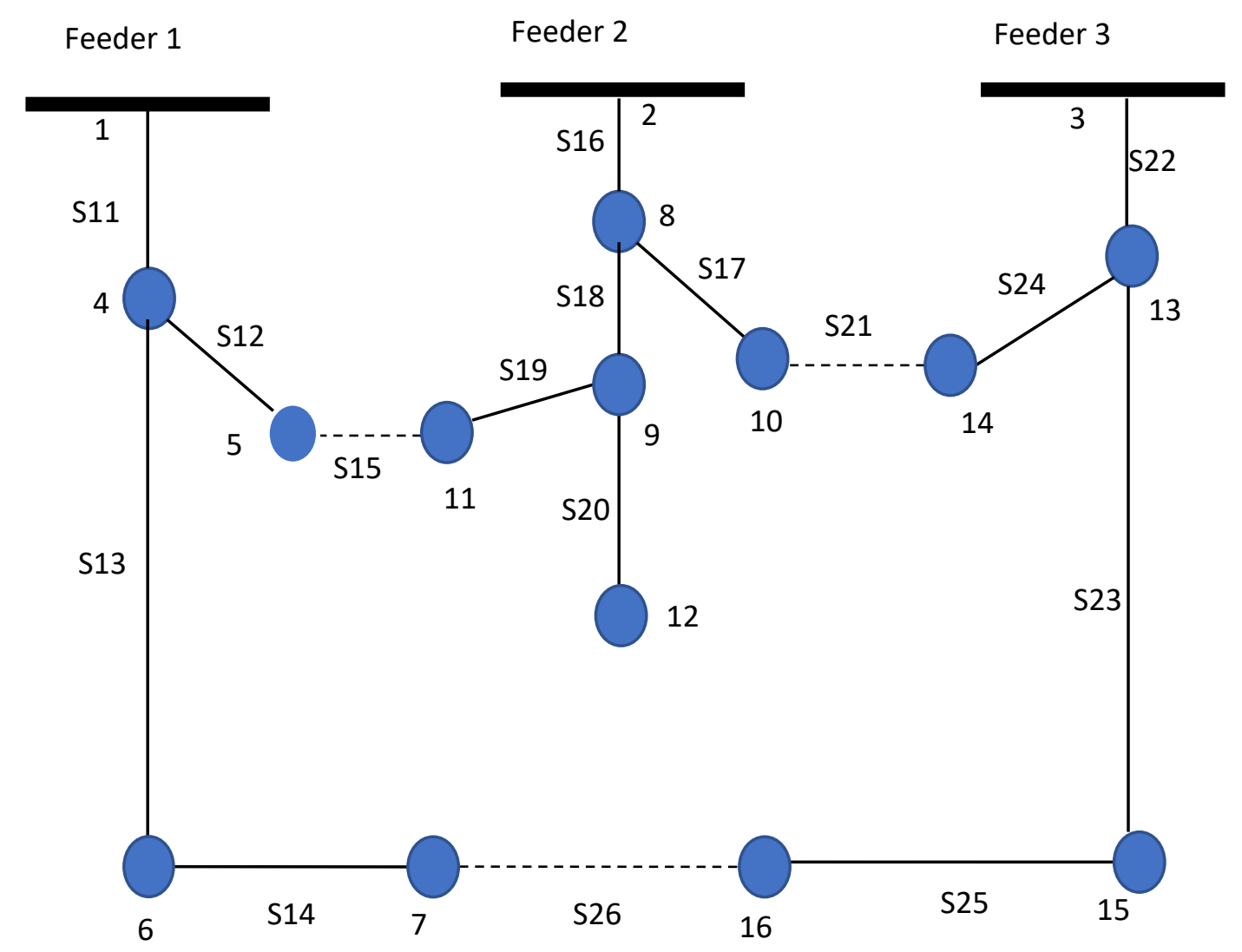

Figure 4.1 Revised IEEE 16-bus test system

The minimizing loss problem is known as:

$$
\operatorname{minP}_{\text {loss }}\left(S_{v}\right)
$$

With

$$
P_{\text {loss }}=\sum_{i=1}^{N b} R_{i} *\left|I_{i}\right|^{2}
$$

Such that,

$$
\begin{gathered}
I_{i} \leq I_{\max } \quad \forall i=1 \sim N b \\
V_{\text {min }} \leq V_{j} \leq V_{\text {max }} \quad \forall j=1 \sim N
\end{gathered}
$$

Where, 
$P_{\text {loss }}=$ total line losses of the distribution feeder

$S_{v}=$ the status of the switch

$\mathrm{Nb}=$ total number of branches in the system

$V_{\min }, V_{\max }=$ minimum and maximum voltage magnitude limit of the node voltage

$R_{i}=$ the resistance of branch $i$.

$I_{i}=$ the current magnitude of branch $i$ ( the branch current is equal to the sum of all load currents beyonde a particular branch).

$N=$ total number of buses.

The planning solution algorithm is to follow the solution of the simple genetic algorithm (SGA) [61] and refined genetic algorithm (RGA) [62]. The novelty of the proposed method herein is that the formulation of the population matrix is different and the tabu search is used to prevent from evaluating the same chromosome more than ones. Defying the upper and lower bounds of each loop of the meshed system is the main key to reducing the size of the population matrix. Besides, this will guaranty that all the chromosomes of the matrix become unique and finding the optimal solution will be easier and fast more or less system converges fast and less computation time. After the convergence, strings are decoded to the original solution variables and the solutions are obtained.

The assumption herein is that the load type is considred to be a $P Q$ load and provided at the end node. By following the soultion methodolgy presebted in the previous chpter, despite the size of the system even with the lack of information about the given system the only data required here is the line and load data. Line data provides the impedance of each branch and also the connectivity of the adjacent nodes. Load data provides the load power at each end node bus.

\subsection{Encoding and initialization techniques of FGA}

In this chapter, the total number of meshes $m$ in the system is formulated based on the total number of normally opened tie switches $S$. To show the status of $\mathrm{S}$ switches in a distribution feeder, a string of $S$ bit can be used. For each switch, "1" shows that the switch is closed and " 0 " shows it is open. If the status of switches change, their string code will change too. Then reconfiguration problem is a binary issue, because all states of the network are combinations of 0 and 1 . An optimum code for the network has to be found 
which results in a minimum value of the objective function. Basically, for a distribution network with $S$ switches, there are $2^{S}$ possible codes which all of them are not necessarily feasible. For the infeasible solutions, since its predictable in the reconfiguration problems, it will be saved in the Tabu list to limit both the processing time and searching space( e.g., not to be evalated more than onse). To determine the feasible states let's consider the network shown in Figure 4.1, it contains three feeders and based on the radiality concern, it is obvious that there must not be any closed loop between these feeders, and all the loads must be energized too. So, for each mesh we should have only one 0 for each network code (e.g., only one open switch), if there are more, that code is infeasible. The other important limitation is that the main feeders (directly connected to the sub-station) or switches of end loads or feeders should always be 1. For the illustration purpose, see Figure 4.1. There are three meshes, mesh 1 is the left branches (codes 12,15,19, and 18), mesh 2 is the right branches (codes 17, 21 and 24), and mesh 3 is the down branches (codes 13, 14, 26, 25, and 23). As mentioned above, there should be only one 0 in every three codes to maintain structure feasibility, the total number of feasible codes is equal to the multiplication of the number of all collected codes.

The assumption as mentioned above is that there should be no common switches between any two meshes and also end node's switches must always remain closed and excluded from the mesh structures (e.g., node 12 in figure 4.1). Table 4.1 illustrating the coding scheme of the proposed algorithm, where ' 0 ' indicates an open switch, ' 1 ' indicates a closed switch. $M 1$ indicated mesh number 1 , and $M 2, M 3$ are mesh 2 and 3 respectively. Notation $X$ represents the chromosomes of the population matrix. The genes of each chromosome are representing the open switches. For instance, the chromosome $X_{\text {base }}=$ $\left[w_{1}, w_{2}, w_{3}\right]=[15,21,26]$, where 15,21 , and 26 are the genes of this chromosome, and represents the open switches of three meshes respectively. Basically, based on the proposed method there should be no common of any genes of each chromosome, this advantage will improve the performance of GA and speed the system convergence. Taking into account that the genes herein is an integer numbers and are not binary in contrast to traditional GA. The boundary of each variable is set to be from 1 to the total number of switches of each mesh. Then, transformation code is needed to pass theswitch number to the FGA code for each iteration. Finally, when the system converges the coded switch numbers are decoded 
and transformed back to the actual switch numbers. For example, from table 4.1 $M 1_{\text {proposed }}=[11,12,15,19,18,16]$, which has 6 switches, therefore, the boundaries are set from 1 to 6 , such that $B 1=[1,2,3,4,5,6]$ where $B$ indicates the boundaries of each mesh. Since, in $M 1_{\text {proposed }}$ the switch numbers are non-consecutive so $M 1_{\text {proposed }}$ and $B 1$ must be symmetrical. The same procedure is followed for the other meshes.

Table 4-1 A coding scheme of the test feeder in figure 4.1

\begin{tabular}{|c|c|c|c|c|c|c|c|c|c|c|c|c|c|c|c|c|c|}
\hline \multicolumn{2}{|c|}{ Base case } & 11 & 12 & 13 & 14 & 15 & 16 & 17 & 18 & 19 & 20 & 21 & 22 & 23 & 24 & 25 & 26 \\
\hline \multicolumn{2}{|c|}{$\begin{array}{c}\text { S base (switch } \\
\text { status) }\end{array}$} & 1 & 1 & 1 & 1 & 0 & 1 & 1 & 1 & 1 & 1 & 0 & 1 & 1 & 1 & 1 & 0 \\
\hline \multirow{3}{*}{ 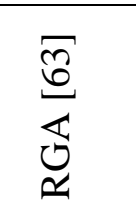 } & M1 & 11 & 12 & 15 & 19 & 18 & 16 & & & & & & & & & & \\
\hline & M2 & 16 & 17 & 21 & 24 & 22 & & & & & & & & & & & \\
\hline & M3 & 13 & 14 & 26 & 25 & 23 & 24 & 21 & 17 & 18 & 19 & 15 & 12 & & & & \\
\hline \multirow{3}{*}{ 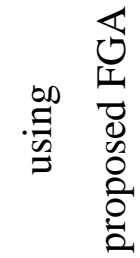 } & M1 & 11 & 12 & 15 & 19 & 18 & 16 & & & & & & & & & & \\
\hline & M2 & 17 & 21 & 24 & 22 & & & & & & & & & & & & \\
\hline & M3 & 13 & 14 & 26 & 25 & 23 & & & & & & & & & & & \\
\hline
\end{tabular}

Genetic operators are the stochastic transition rules applied to each chromosome during each generation procedure to generate a newly improved population from the previous one. A genetic algorithm usually consists of reproduction, crossover and mutation operators. GA highly relies on the crossover operator.

\subsection{Initialization}

The initial population is based on the number of variables and the population size $(p)$. Thus, the initial population is $\left\{X_{i} \mid i=1,2, \ldots \ldots, p\right\}$.

From table 4.1 $X_{i}=[L B, U B]$ where, $X_{1}=[1,6], X_{2}=[1,4]$, and $X_{3}=[1,5]$. The proposed topology will randmly chose one element from each loop and the population matrix is fromulated as

$$
\text { Pop }_{\text {matrix }}=\left[\begin{array}{c}
\mathrm{x}_{1}, \mathrm{x}_{2}, \ldots \mathrm{x}_{\mathrm{n}} \\
: \\
\vdots \\
\mathrm{x}_{1 \mathrm{p}}, \mathrm{x}_{2 \mathrm{p}}, \ldots \mathrm{x}_{\mathrm{np}}
\end{array}\right]
$$


One advantage of the proposed algorithm is that there should not be any similar chromosomes in the population matrix and, each chromosome is evaluated only ones.

\subsection{Crossover}

Crossover is a structured recombination operation by exchanging genes of two parents. In the planned FGA the neighboring genes (switches) has a higher priority to be selected than the other switches in the same mesh. In other words, in Figure 4.1, switch s24 and s17 have higher priorities to replace switch s21. The new chromosomes obtained from the crossover and mutation is formulating the offspring. The probability of parent-chromosomes crossover is assumed to be 0.8 .

\subsection{Mutation}

Mutation is the occasional random alteration of genes. Also, it is the process of random modification of a string position by changing " 0 " to " 1 " or vice versa, with a small probability. It prevents the loss of genes through reproduction and crossover by ensuring that the probability of searching any region in the problem space is never zero. The probability of mutation is assumed to be 0.02 .

\subsection{Fitness Function}

In order to formulate the objective function (4.1) will be used as a fitness function by adding constraints as:

$$
\mathrm{F}=\mathrm{P}_{\mathrm{loss}}\left(\mathrm{S}_{\mathrm{V}}\right)+\sum_{\mathrm{i}=1}^{\mathrm{Nb}} \lambda_{\mathrm{l}, \mathrm{i}} *\left(\mathrm{I}_{\mathrm{i}}-\mathrm{I}_{\mathrm{lim}}\right)^{2}+\sum_{\mathrm{j}=1}^{\mathrm{N}} \lambda_{\mathrm{V}, \mathrm{j}} *\left(\mathrm{~V}_{\mathrm{j}}-\mathrm{V}_{\mathrm{lim}}\right)^{2}
$$

Where, $\lambda_{I, i}$, and $\lambda_{V, j}$ are the penalty factors that can be adjusted in the optimisation procedure. $I_{\text {lim }}$ and $V_{\text {lim }}$ are defined as:

$$
\begin{gathered}
I_{\lim }= \begin{cases}I_{i} & \text { if } I_{i} \leq I_{\max } \\
I_{\text {max }} & \text { if } I_{i}>I_{\text {max }}\end{cases} \\
V_{\lim }= \begin{cases}V_{j} & \text { if } V_{\text {min }} \leq V_{j} \leq V_{\text {max }} \\
V_{\text {min }} & \text { if } V_{j}<V_{\text {max }} \\
V_{\text {max }} & \text { if } V_{j}>V_{\text {max }}\end{cases}
\end{gathered}
$$


The idea herein is that if one or more variables violate their limits, the penalty factors will increase, and the corresponding chromosome will be entered into the Tabu list to avoid generating the same infeasible solution again. 


\subsection{FGA flowchart}

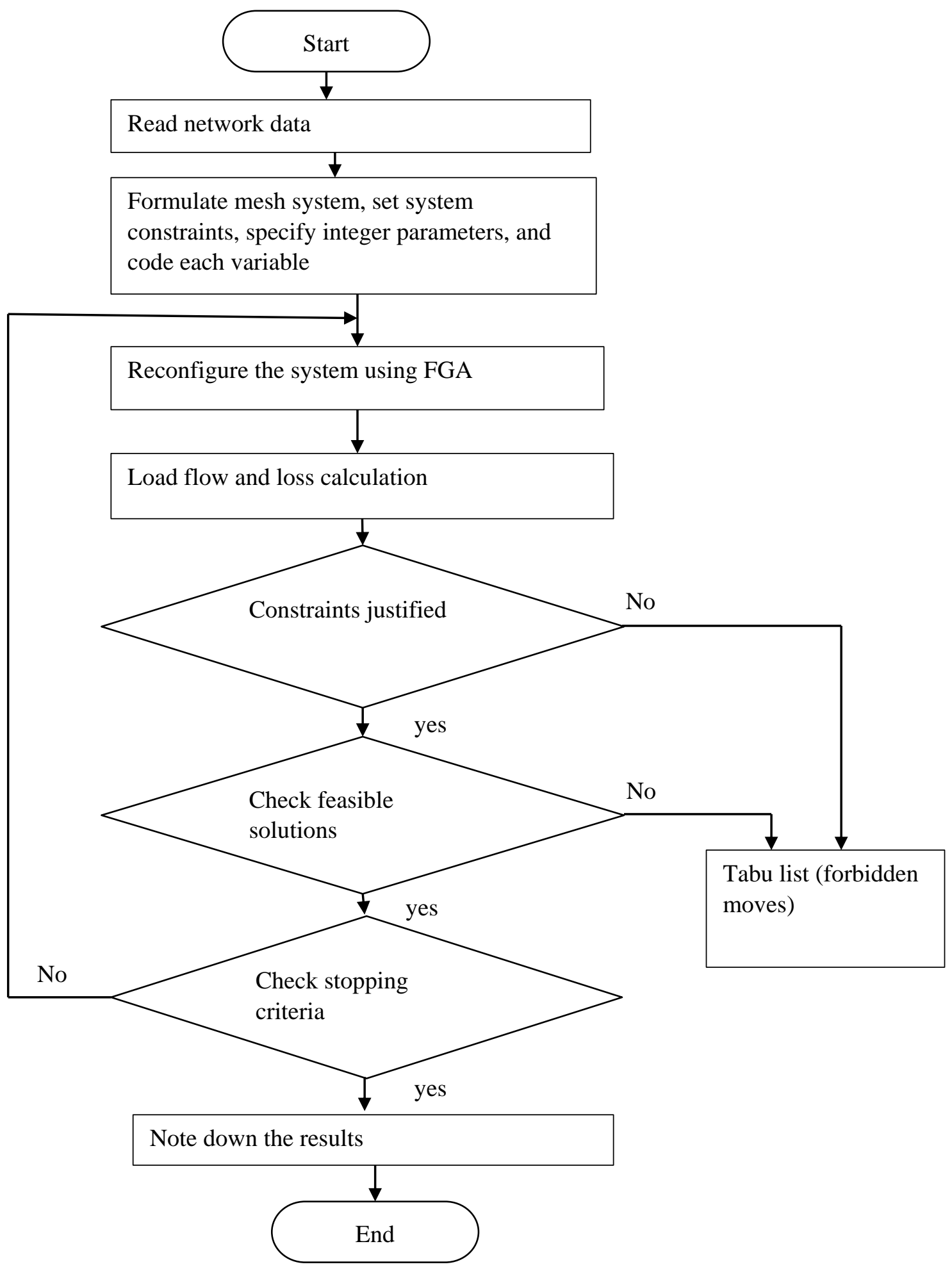

Figure 4.2 Flowchart of the proposed FGA 


\subsection{Simulation results and discussions}

The proposed FGA is tested on a revised 16-bus IEEE distribution test system shown in figure 4.1. The assumption to be made is that each transformer can serve all the system loads. Distribution feeder reconfiguration is implemented when the load is changed or when the fault occurs in the system. The simulation of the proposed algorithm is performed using different steps and employing the 3D matrix method to solve different system functions. Also, since the parameters of the fitness function are an integer, the proposed FGA code can handle all the data types. In this research, the strategy of the encoding and decoding are the main parameters to be considered.

System constraints are considered to be the node voltage boundaries and the branch currents limits. Based on the literature review, the node voltages are demonstrated to be between 0.95 and 1.05 p.u. Additionally, it is desirable that the branch currents of the system be less or equal to their respective rated capacity. A \%15 overloading is allowed for each branch [5].

The stopping criteria to be considered in here is that the process of generating new trails with the best fitness will be continued until the fitness value are optimized, or the maximum generation number is reached. the strategy and the implementation of the FGA process are illustrating in the flowchart of figure 4.2. One can also add another stopping rules such that the population matrix is to be updated after each iteration. Additionally, for each generated parents (e.g., new chromosomes ) the Tabu list is checked to ensure that the new child is not in the Tube list in this way, it is to be guaranteed that each chromosome is evaluated ones and also the convergence speed is improved. This research is mainly focusing on optimally configuring the radial distribution system for power loss reduction and voltage profile improvement. Different criteria can be used and also different objectives can be solved using the proposed algorithm.

The test system presented in figure 4.1 is used to validate the performance of the proposed method. The system contains three transformers sited as node 1,2 and 3 respectively. The assumption as mentioned above is that each transformer should be able to serve the whole system in case of any contingency. The system also has 13 normally closed switches and three normally open tie switches. The total loads are 31.7 MW and 20.3 MVAr, base power is $100 \mathrm{MVA}$, and the base voltage is $12.66 \mathrm{kV}$. 
Table 4-2 The simulation parameters of FGA

\begin{tabular}{|l|l|l|l|}
\hline Number of variables & $\begin{array}{l}\text { Maximum } \\
\text { generation }\end{array}$ & Mutation probability & $\begin{array}{l}\text { Crossover } \\
\text { probability }\end{array}$ \\
\hline 3 & 200 & 0.02 & 0.8 \\
\hline
\end{tabular}

Table 4.2 shows the simulation parameters of the proposed method. The maximum generation is meant to be high to expand the search area and also to ensure finding the optimum solution.

The load flow analysis presented in chapter 3 is used to investigate the performance of the FGA. Because the GA is a stochastic optimization method, the optimization results and calculation time of each test are different.

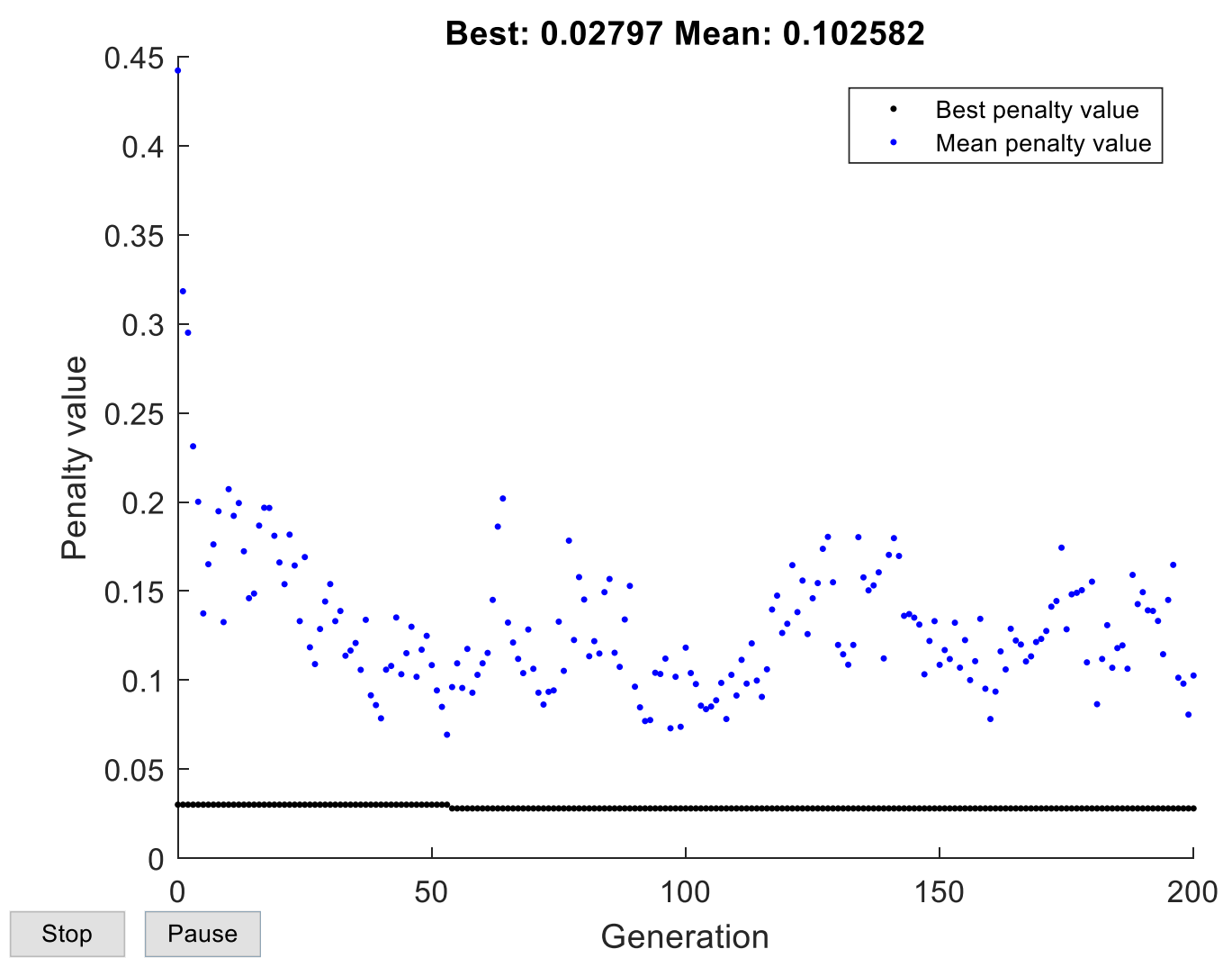

Figure 4.3 The proposed FGA performance characteristics of the best penalty value and mean value of the fitness function 
Although the size of the population matrix is 150 by 3 , the proposed method since it is using the Tabu list will first check and eliminate the repeated chromosomes from the population matrix and then start the evaluation process. For this particular example or test system, there are only 83 survival chromosomes from the total of 150 , this means that there will be no repeated process of any chromosomes. figure 4.3 represents the performance of the FGA the initial penalty is 10 , and the penalty factor is 100 .

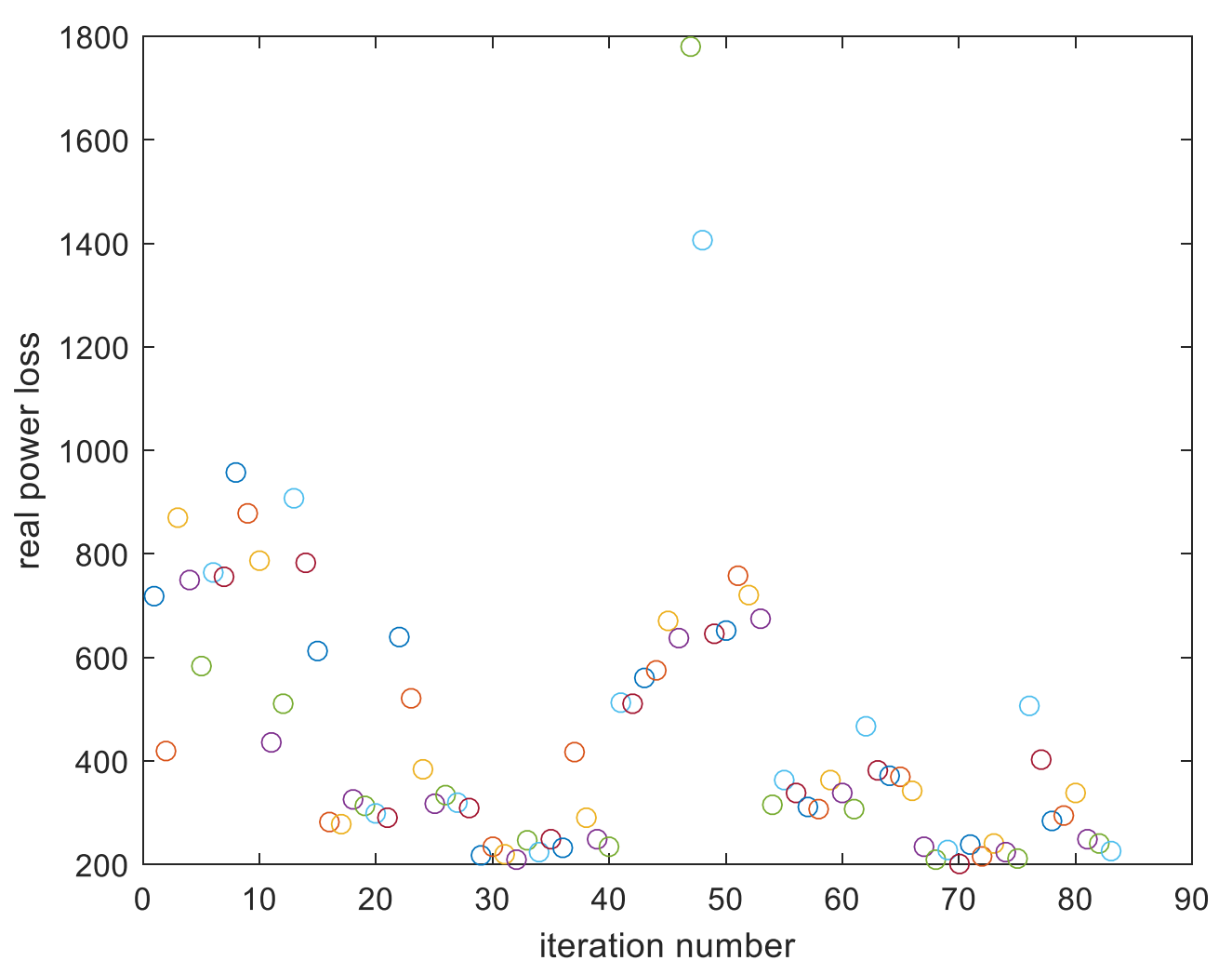

Figure 4.4 The real power loss computation using the proposed method

Figure 4.4 shows the system total real power loss. This optimal result is obtained with optimal switch operation of opening the switches s15, s21 and s14. One advantage of the genetic algorithm is to search from a population of points rather than a single point. Therefore, the obtained result should be promising. figure 4.5 is a zoomed layout of figure 4.4 to show the exact value of the system total real power loss. By the way, the system total real power loss before reconfiguration is $220 \mathrm{~kW}$ and $256.6641 \mathrm{kVAr}$. from figure 4.5 , it can be noticed that the real power loss reduction is $18.1 \mathrm{~kW}$, i.e., $8.22 \%$ real power loss reduction after reconfiguring the system. 


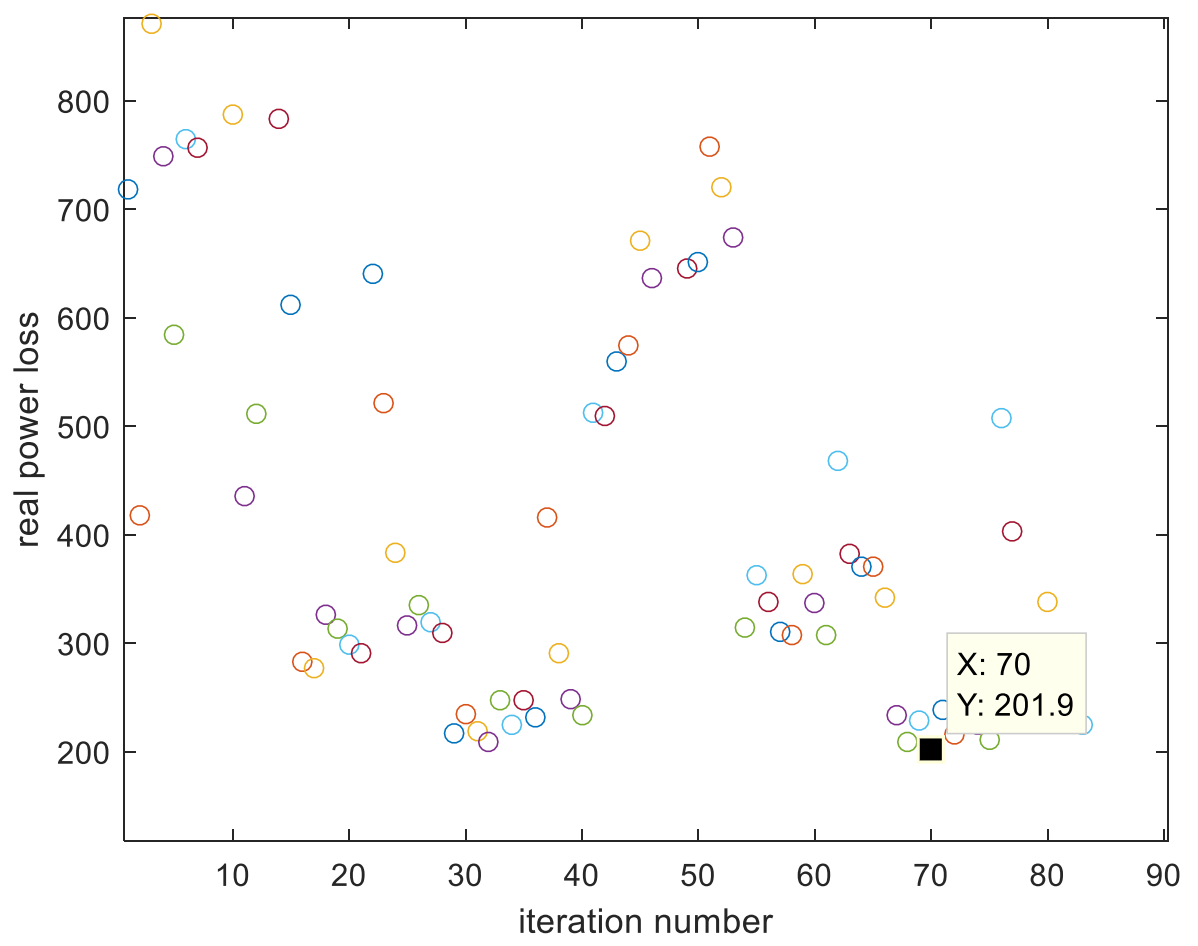

Figure 4.5 Zoomed in the plot for real power loss value

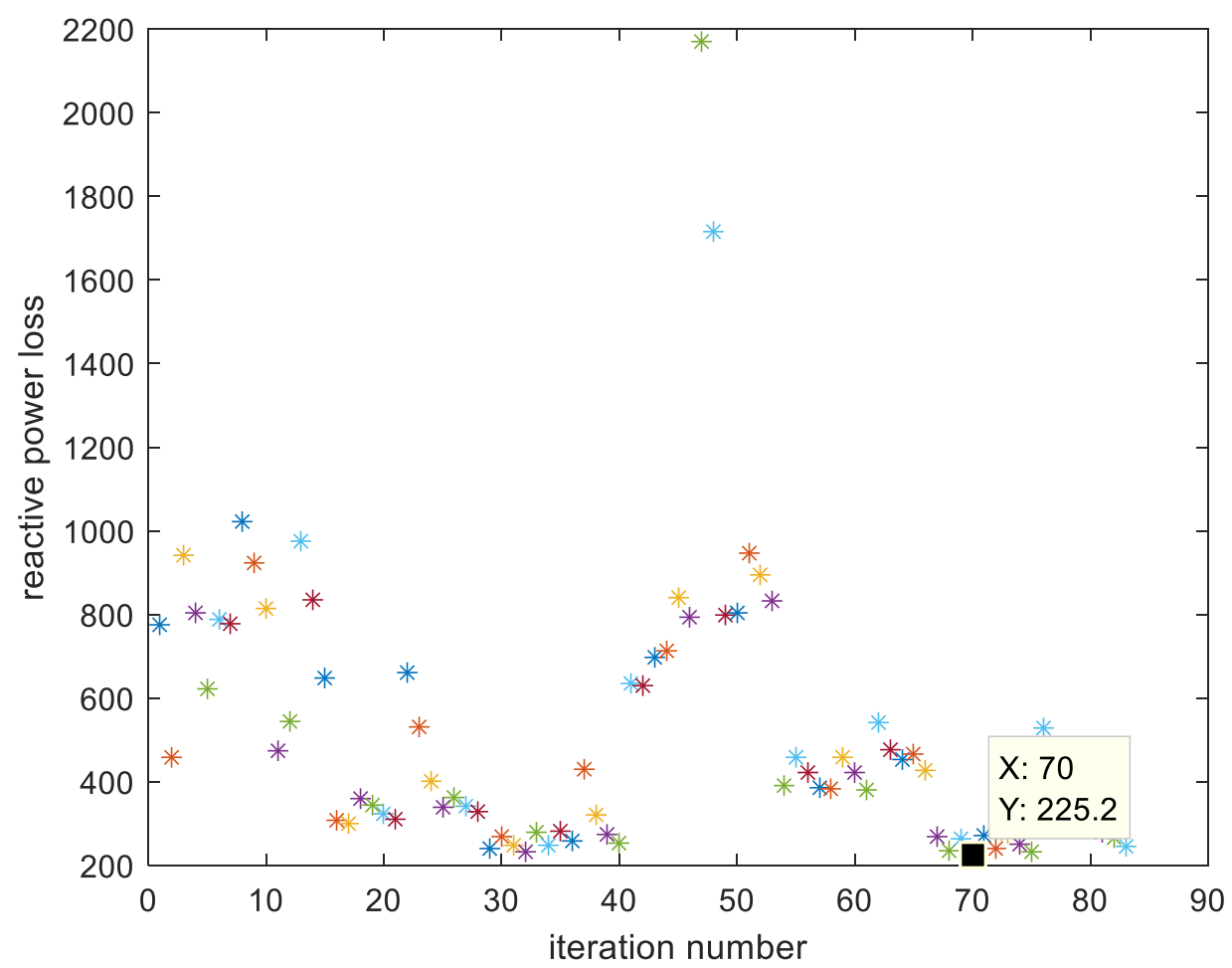

Figure 4.6 Reactive power loss of the test system using FGA 
Figure 4.6 represents the total reactive power loss of the test system. It can be seen that the reactive power loss is $225.2 \mathrm{kVAr}$. This means the reactive power loss reduction is 31.4640 $\mathrm{kVAr}$, i.e., $12.25 \%$ power loss reduction.

Table 4-3 The comparison of the proposed FGA with other methods

\begin{tabular}{|l|l|l|l|l|l|l|l|l|l|}
\hline $\begin{array}{l}\text { Topology } \\
\text { name }\end{array}$ & $\begin{array}{l}\text { Total } \\
\text { active } \\
\text { load } \\
\text { MW }\end{array}$ & $\begin{array}{l}\text { Total } \\
\text { reactiv } \\
\text { e load } \\
\text { MVAr }\end{array}$ & $\begin{array}{l}\text { Base } \\
\text { voltage }\end{array}$ & $\begin{array}{l}\text { Initial } \\
\text { real } \\
\text { power } \\
\text { loss } \\
\mathrm{kW}\end{array}$ & $\begin{array}{l}\text { Initially } \\
\text { opened } \\
\text { switching }\end{array}$ & $\begin{array}{l}\text { Obtained } \\
\text { results } \\
\text { (real } \\
\text { power } \\
\text { loss kW) }\end{array}$ & $\begin{array}{l}\text { Optimal } \\
\text { switching }\end{array}$ & $\begin{array}{l}\text { Populati } \\
\text { on/gene } \\
\text { ration }\end{array}$ & $\begin{array}{l}\text { Elapsed } \\
\text { time } \\
(\mathrm{sec})\end{array}$ \\
\hline Das [64] & 4.47 & 3.06 & 11 & 5.09 & $\begin{array}{l}33,34,35 \\
, 36,37\end{array}$ & 4.32 & $\begin{array}{l}8,27,33, \\
34,36\end{array}$ & $70 / 100$ & 1900 \\
\hline Civanlar & 28.7 & 17.3 & 23 & 1.79 & $17,18,19$ & 1.63 & $10,11,19$ & $30 / 40$ & 30 \\
\hline Baran & 3.72 & 2.29 & 12.66 & 4.13 & $\begin{array}{l}33,34,35 \\
, 36,37\end{array}$ & 3.56 & $\begin{array}{l}7,9,14,3 \\
2,37\end{array}$ & $40 / 50$ & 160 \\
\hline $\begin{array}{l}\text { Propossed } \\
\text { FGA }\end{array}$ & 31.7 & 20.3 & 12.66 & 9.93 & $15,21,26$ & 2.74 & $15,21,14$ & $30 / 40$ & 18.6 \\
\hline
\end{tabular}

Table 4.3 represents the comparison of the proposed method with other topologies. More data can be discussed from the simulations process, in this study the only focus was the minimization of power losses and voltage profile improvement. Also from the table, it can be seen that the elapsed time is way less using the proposed algorithm because each chromosome is evaluated only ones and the topology ensured that both the repeated and infeasible solutions are saved in the Tabu list.

\subsection{Conclusion}

This chapter has presented an improved smart technique of configuring the radial distribution system based on FGA for the minimization of the system power losses and improving its voltage profile. The problem is formulated as a multi-objective problem solved using the proposed method and the graph theory together with a backward-forward load flow method presented in chapter 3. The graph theory is used in order to generalize and obtain the radial connectivity of the test system (e.g., to make sure that all the loads are served and the radiality is retained). The FGA is like the traditional GA in relying on the crossover and mutation process. However, FGA is using integer parameters in which the decoding and coding of the fitness function are unique in contrast with other methods. 
Additionally, FGA solutions do not rely on the initial population values because it searches from a population of points and the feasible obtained results are compared, and the optimal result is chosen in each iteration processes. The feasible solution is defined as the solution of which all the operational constraints are satisfied (e.g., the branch currents are at or below the limits, and the node voltages are within the specified boundaries). Switching status or infeasible solution are not carried out. Basically, only feasible solutions are crossed or mutated at each iteration step.

In comparison with other methods ( references), the detailed coding strategy and FGA procedures are valid and effective for all networks even with an all parameter types (e.g., binary, integer, etc.). the obtained simulation results have shown the effectiveness of the proposed method. The computation times are way less compared with other references, that is because of the way the population matrix constructed and based on the partially meshed networks. 


\section{Chapter 5}

\section{Optimal Distribution Feeder Reconfiguration with Distributed Generation using Differential Evolution (DE)}

\subsection{Introduction}

In this chapter, the radial distribution feeder reconfiguration for loss reduction and voltage profile improvement including the presence of DGs using the Differential Evolution DE is proposed. The DGs units are assumed to exist in the system which means its location and size is not controllable since it is a customer owned. DGs can harm the grid if placed or connected randomly resulting in increasing the end user voltage. The study here is aiming to keep the system node voltages within an acceptable limit by configuring the system using DE and checking the voltage violation limit for each load flow execution. The proposed DE algorithm is a new approach used in a power system area. DE is a stochastic, population-based search strategy developed by Storn and Price [53]. While DE shares similarities with other evolutionary algorithms (EA), it differs significantly in the sense that distance and direction information from the current population is used to guide the search process (e.g., using the current population). In DE algorithm, all solutions have an equal opportunity of being preferred as parents, and selection does not depend on their fitness values. Nowadays, the integration of renewable energy sources adds more complexity to the optimization problem that cannot be easily solved with existing classical mathematical optimization methods. DE performance depends on two processes, known as the mutation process and recombination (crossover) process. The two tuning parameters, called scale factor $(\mathrm{F})$ and crossover probability $(\mathrm{CR})$, which control the performance of $\mathrm{DE}$ in its mutation and recombination processes.

In $\mathrm{DE}$ operation the child vector is generated by applying the mutation and crossover operation. In mutation operation, a trial vector is generated with the help of the objective vector and two erratically preferred individuals. The deviation in the objective vector depends on mutation factor $\mathrm{F}$ and the difference between the randomly selected individuals. The crossover operation is applied between the objective vector and parent vector to generate the child vector using crossover probability (CR). Like any other method, these 
tuning factors have a boundary such that large values may result in skipping of actual solutions, and small values also may cause performance degrades. This makes it clear that the conflicts in the child vector from the parent vector depends on the values of $F$ and $C R$. In this chapter, the population matrix size is formulated in such a way the rows represent the number of iteration time, and the column is representing the number of variables (e.g., number of tie switches). Each chromosome of the population matrix contains a number of open switches called genes, each gene representing switch number. For example, s15, s21, and s26 are the genes of this chromosome and represents the open switches in three meshes, respectively, as shown in Figure 5.1. The effectiveness of the proposed algorithm is tested on two systems, IEEE 16 and 33-bus radial distribution system having three substations and one substation respectively. The proposed algorithm is finally compared to some previous methods such as a Genetic algorithm.

The remainder of this chapter is described as follows. Section 5.2 introduces problem formulation using 16-bus test feeder for illustration propose. Section 5.3 presents the proposed Differential Evolution algorithm DE. Section 5.4 discusses the DE operations and tests. The flow chart of the DE algorithm is represented in section 5.5. test systems descriptions are viewed in section 5.6 two radial distribution test system are been used in this chapter. Simulation results and discussion of both test systems are given in section 5.7. Conclusions are drawn in section 5.8.

\subsection{Problem Formulation}

The complexity of any optimization problem depends on the objective function formulation and the considered constraints. For modem urban distribution systems, the number of distribution transformers may reach two to three thousand, and each transformer may be supplied by four or five different feeders and substations [65]. Such systems are very complex, difficult to monitor, and challenging to control optimally in real-time. Losses associated with each configuration must be calculated, and this requires optimal load flow. The problem is compounded by the desire to maintain the radial configuration of the distribution system and by operational constraints (e.g., voltage range, current limit, etc.) ensuring feeders and transformers are not overloaded and ensuring voltage drop limitations 
are not exceeded. As well, there is a need for efficient data structures and algorithms that will permit reconfiguration in real-time.

In this chapter, the reconfiguration problem is illustrated using the three feeder test system as shown in Figure 5.1. Data for this system can be found in [11]. This sample distribution system contains three feeders with thirteen normally closed sectionalizing switches and three normally opened tie switches, namely s15, s21, and s26.

The loss minimization problem is formulated as:

$$
\min \mathrm{P}_{\text {loss }}(\mathrm{x})
$$

with

$$
\mathrm{P}_{\text {loss }}=\sum_{\mathrm{i}=1}^{\mathrm{Nb}} \mathrm{R}_{\mathrm{i}} *\left|\mathrm{I}_{\mathrm{i}}\right|^{2}
$$

such that,

$$
\begin{gathered}
I_{i} \leq I_{\max } \quad \forall i=1,2 \ldots, N b \\
V_{\text {min }} \leq V_{j} \leq V_{\text {max }} \quad \forall j=1,2, \ldots, N
\end{gathered}
$$

where, $P_{\text {loss }}=$ total line losses of the feeder

$x=$ status vector of the switch

$N b=$ total number of branches in the whole system

$I_{i}=$ current magnitude of branch $i$

$I_{\text {max }}=$ upper limit of branch current magnitude

$V_{j}=$ voltage magnitude of branch $j$

$V_{\min }, V_{\max }=$ lower and upper limit of node voltage magnitude

$R_{i}=$ resistance of branch $i$

$N=$ total number of buses

$x=\left[s w_{1} s w_{2} s w_{3}, \ldots s w_{\text {number of vairables }]}\right.$

From Figure $5.1 x=\left[\begin{array}{ll}15 & 21\end{array}\right.$ 26], these represents the open switches of three meshes, respectively. One advantage of the proposed method is using the integer numbers instead of binary numbers involving several modifications of the basic algorithm. 


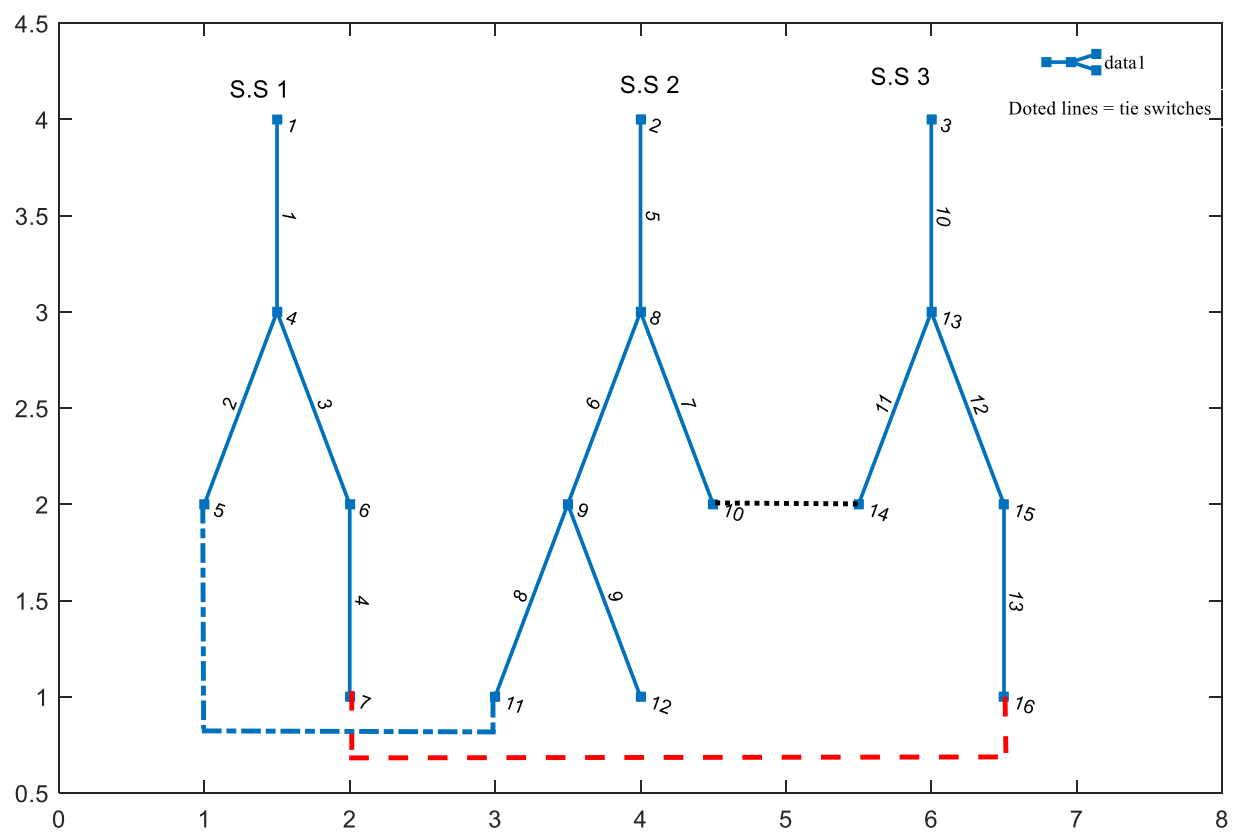

Figure 5.1 Revised IEEE 16-bus test system

Distribution system losses are known as $I^{2} R$, and thus the problem is a nonlinear integer optimization problem, with a quadratic objective function, $0-1$ type state variables where 0 indicates open switch and 1 represents a closed switch, and linear constraint equations with state-dependent constraint formula. The value of the objective function is determined from the optimal power flow solution given settings of the control variables. At each iteration, a new power flow is required to determine a new system operating point. The problem presents a heavy computational burden for even a moderately-sized distribution system.

Reconfiguration for loss reduction typically involves evaluating many combinations of switching options to determine which option offers the lowest losses. Apparently, in a large system, even with a high-speed computer, the time needed to complete a load flow to evaluate every option would be prohibitive. 


\subsection{The Proposed Differential Evolution Algorithm (DEA)}

Differential evolution is a strategy that optimizes a problem by iteratively trying to enhance an individual solution with regard to a specified gauge of excellence. DE algorithm is used for multidimensional real-valued functions, but it does not put together the ascent of the problem being optimized, so the optimization problem does not have to be differentiable as is mandatory for traditional optimization methods [66]. DE algorithm optimizes a problem by considering a population of candidate solutions and generating new contestant solutions by combining existing ones according to its simple formulae, and then memorizing whichever candidate solution has the minimum score fitness on the optimization problem at hand. Thus in this way, the optimization problem is treated as a black box that merely makes available a gauge of quality specified a candidate solution, and the gradient is for that reason not considered necessary.

\subsubsection{Overview of the optimization parameters}

In simple terms, optimization is the attempt to maximize a system's desirable properties while simultaneously minimizing its undesirable characteristics. What these properties are and how effectively they can be improved depends on the problem at hand. In general, the objective function, $f(x)=f\left(x_{0}, x_{1}, \ldots, x_{D}-1\right)$, has $\mathrm{D}$ parameters that influence the property being optimized. There is no unique way to classify objective functions, but some of the objective function attributes that affect an optimizer's performance are:

- Parameter quantization: is to check whether the objective function variables continuous, discrete or do they belong to a finite set. Additionally, are all the variables of the same type.

- Parameter dependence: Can the objective function parameters be optimized independently (separable function), or does the minimum of one or more parameters depend on the value of one or more other parameters (parameterdependent function).

- Dimensionality, D: How many variables define the objective function.

- Modality: Does the objective function have just one local minimum (uni-modal) or more than one (multi-modal)? 
- Constraints: Is the function unconstrained, or is it subject to additional equality and inequality constraints?

General formulation of DE:

DE differs from other algorithms such that:

- mutation is applied first to generate a trial vector, which is then used within the crossover operator to produce one offspring,

- mutation step sizes are not sampled from a prior known probability distribution function.

- In DE, mutation step sizes are influenced by differences between individuals of the current population.

\subsubsection{Parameter Representation}

DE encodes all parameters as floating-point numbers, regardless of their type. Even integer and discrete variables are encoded as real values to add diversity to their difference distributions DE have several significant advantages over the traditional GA approach to continuous parameter optimization. Advantages include:

- Ease of use

- Efficient memory utilization

- Lower computational complexity - scales better on large problems

- Lower computational effort - faster convergence

- Greater freedom in designing a mutation distribution.

\subsubsection{Population Structure}

In addition to the population size, $N_{p}$, the performance of DE is influenced by two control parameters, the scale factor, $F$, and the probability of recombination, $C R$. The more individuals there are in the population, the more differential vectors are available, and the more directions can be explored. However, it should be kept in mind that the computational complexity per generation increases with the size of the population.

DE's most versatile implementation maintains a pair of vector populations, both of which contain $N_{p}$ (the number of population members) D-dimensional vectors of real-valued parameters. The current population, symbolized by $P_{x}$, is composed of those vectors, $x_{i, g}$, 
that have already been found to be acceptable either as initial points, or by comparison with other vectors.

For an objective function $f: X \subseteq \mathbb{R}^{D} \rightarrow \mathbb{R}$ where the feasible region $X \neq \emptyset$, the minimization problem is to find $x^{*} \in X$ such that

$$
\mathrm{f}\left(\mathrm{x}^{*}\right) \leq \mathrm{f}(\mathrm{x}) \forall \mathrm{x} \in \mathrm{X}
$$

such that: $f\left(x^{*}\right) \neq-\infty$

$$
\begin{gathered}
\mathrm{P}_{\mathrm{x}, \mathrm{g}}=\left(\mathrm{x}_{\mathrm{i}, \mathrm{g}}\right) \\
x_{i, g}=\left[x_{0, i, g}, x_{1, i, g}, \ldots \ldots x_{j, i, g}\right]
\end{gathered}
$$

Where, $i=0,1, \ldots, N_{p}-1, g=0,1, \ldots, g_{\text {max }}$, and $j=0,1, \ldots, D-1$

$N_{p}=$ population size

$g=$ generation number

$D=$ D-dimensional search space

Indices start with 0 to simplify working with arrays and modular arithmetic. The index, $g=0,1, \ldots, g_{\max }$, indicates the generation to which a vector belongs. In addition, each vector is assigned a population index, $i$, which runs from 0 to $N_{p}-1$. Parameters within vectors are indexed with $j$, which runs from 0 to $D-1$.

Once initialized, DE mutates randomly chosen vectors to produce an intermediary population, $P_{v, g}$, of $N_{p}$ mutant vectors, $v_{i, g}$ :

$$
\begin{aligned}
& \mathrm{P}_{\mathrm{v}, \mathrm{g}}=\left(\mathrm{V}_{\mathrm{i}, \mathrm{g}}\right) \\
& V_{i, g}=\left(v_{j, i, g}\right)
\end{aligned}
$$

Each vector in the current population is then recombined with a mutant to produce a trial population, $P_{u, g}$ of $N_{p}$ trial vectors, $u_{i, g}$ :

$$
\begin{aligned}
& \mathrm{P}_{\mathrm{u}, \mathrm{g}}=\left(\mathrm{u}_{\mathrm{i}, \mathrm{g}}\right) \\
& u_{i, g}=\left(u_{j, g}\right)
\end{aligned}
$$

During recombination, trial vectors overwrite the mutant population so that a single array can hold both populations. 


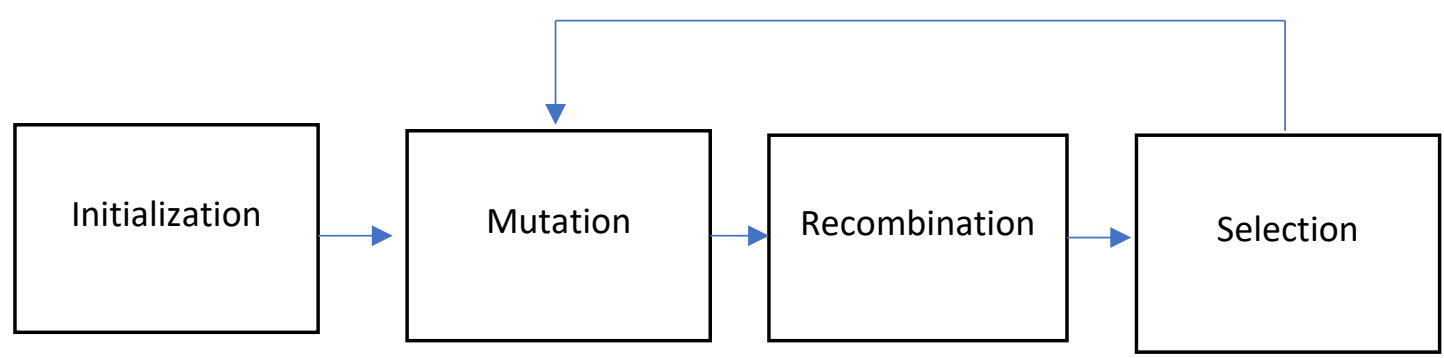

Figure 5.2 General Evolutionary Algorithm Procedure

\subsubsection{Initialization}

Before the population can be initialized, both bounds for each parameter must be specified. These $2 D$ values can be collected into two, $D$-dimensional initialization vectors, $L b$ and, $U b$, for which subscripts $L$ and $U$ indicate the lower and upper bounds, respectively.

$$
\mathrm{x}_{\mathrm{j}}^{\mathrm{L}} \leq \mathrm{x}_{\mathrm{j}, \mathrm{i}, 1} \leq \mathrm{x}_{\mathrm{j}}^{\mathrm{U}}
$$

Once initialization bounds have been specified, a random number is assigned to each parameter of every vector value from within the prescribed range. For example, the initial value $(g=0)$ of the $j^{\text {th }}$ parameter of the $i^{\text {th }}$ vector is:

$$
\mathrm{X}(\mathrm{m}, \mathrm{n})=\mathrm{X} \_\min (\mathrm{n})+\operatorname{round}\left(\operatorname{rand}() *\left(\mathrm{X} \_\max (\mathrm{n})-\mathrm{X} \_\min (\mathrm{n})\right)\right)
$$

The random number generator, rand(), returns a uniformly distributed random number Even if a variable is discrete or integral, it should be initialized with a real value since DE internally treats all variables as floating-point values regardless of their type.

In this dissertation, since most of the parameters are representing the switch status which is an integer value. The parameter's types is an integer and the Matlab function round is used to obtain the intiger values. The upper and lower bounds of each parameter is formulated based on the meshed loop structure. In other words, because different loops might have branches and switches in common, there must be at least only one opened branch in each loop for the final radial structure.

In addition, the structure of the system loops must obey these rules:

1) All feeder section must be served,

2) Radial network structure must be retained, 
3) Node voltage magnitude within bounds,

4) Branch currents also must be within bounds.

5) Substation node and end node loads should not be counted.

\subsubsection{Mutation}

One of the DE advantages is searching from a large search space. The mutation procedure expands the search space. Each of the $N$ parameter vector undergoes mutation, recombination, and selection. Once initialized, DE mutates and recombines the population to produce a population of $N p$ trial vectors. In particular, differential mutation adds a scaled, randomly sampled, vector difference to a third vector. Equation (5.8) and (5.9) shows how to combine three different, randomly chosen vectors $x_{r 1, G}, x_{r 2, G}$ and $x_{r 3, G}$ to create a mutant vector, $v_{i, g}$ :

$$
\mathrm{v}_{\mathrm{i}, \mathrm{g}}=\mathrm{x}_{\mathrm{r} 0, \mathrm{~g}}+\mathrm{F}\left(\mathrm{x}_{\mathrm{r} 1, \mathrm{~g}}-\mathrm{x}_{\mathrm{r} 2, \mathrm{~g}}\right)
$$

The scale factor, $F \in(0, \infty)$, is a positive real number that controls the rate at which the population evolves. While there is no upper limit on $F$, effective values are seldom greater than 1.0 .

Additionally, The scaling factor, $F \in(0, \infty)$, controls the amplification of the differential variations, $\left(\mathrm{x}_{\mathrm{r} 1, \mathrm{~g}}-\mathrm{x}_{\mathrm{r} 2, \mathrm{~g}}\right)$. The smaller the value of $F$, the smaller the mutation step sizes, and the longer it will be for the algorithm to converge. Larger values for $F$ facilitate exploration, but may cause the algorithm to overshoot good optima. The value of $F$ should be small enough to allow differentials to explore tight valleys, and large enough to maintain diversity. As the population size increases, the scaling factor should decrease [3]. The more individuals in the population, the smaller the magnitude of the difference vectors, and the closer individuals will be to one another. Therefore, smaller step sizes can be used to explore local areas. More individuals reduce the need for large mutation step sizes. Empirical results suggest that large values for both $N_{p}$ and $F$ often result in premature convergence [ [67], [68]], and that $F=0.5$ generally provides good performance [ [69], [70], [71]].

The base vector index, $r_{0}$, can be determined in a variety of ways, but for now it is assumed to be a randomly chosen vector index that is different from the target vector index, $i$. Except for being distinct from each other and from both the base and target vector indices, the 
difference vector indices, $r_{1}$ and $r_{2}$, are also randomly selected once per mutant. Figure 5.2 illustrates how to construct the mutant, $v_{i, g}$, in a two-dimensional parameter space. In general, Different approaches can be used to select the target vector and to calculate differentials.

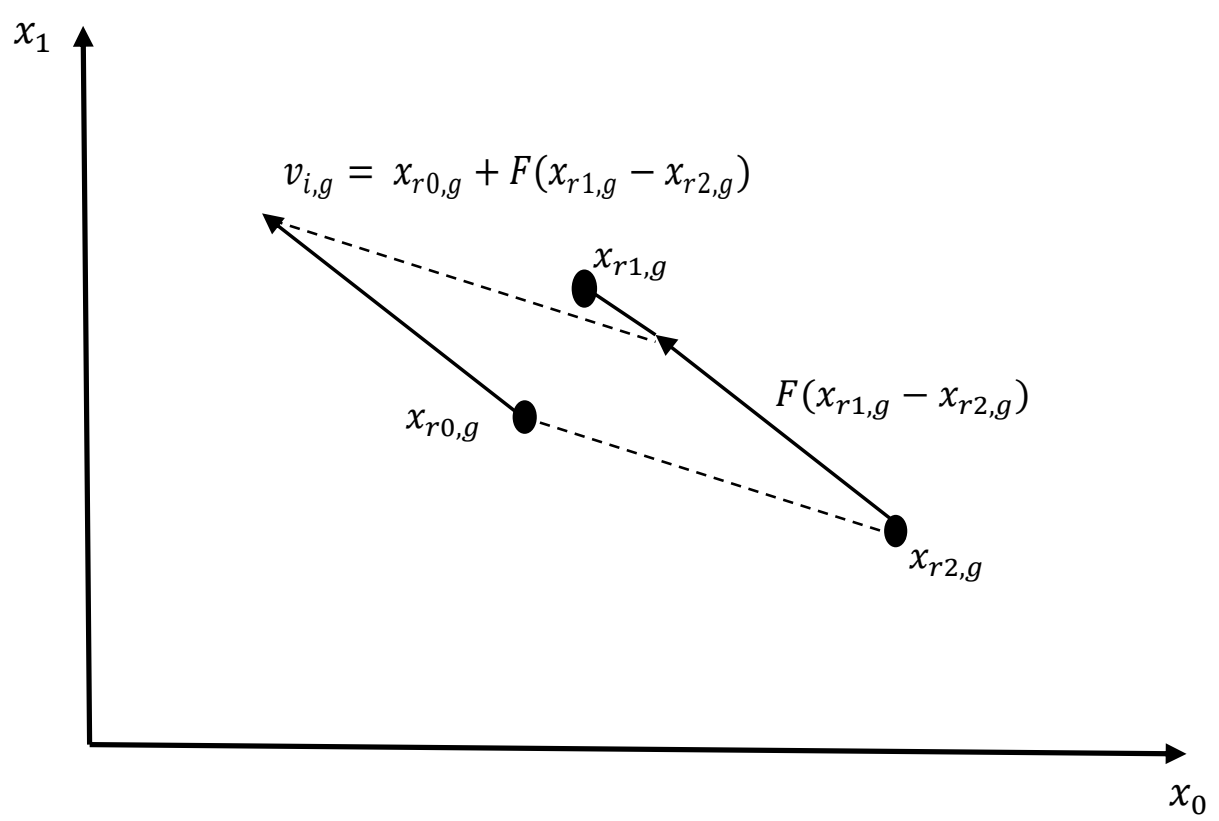

Figure 5.3 Differential mutation: the weighted differential, $\mathrm{F}\left(\mathrm{x} \_(\mathrm{r} 1, \mathrm{~g})-\mathrm{x} \_(\mathrm{r} 2, \mathrm{~g})\right)$ is added to the base vector, $x_{-}(r 0, g)$, to produce a mutant, $v_{-}(i, g)$.

\subsubsection{Recombination}

The probability of recombination, $C R$, has a direct influence on the diversity of DE. This parameter controls the number of elements of the parent, $X_{i, g}$ that will change. The higher the probability of recombination, the more variation is introduced in the new population, thereby increasing diversity and increasing exploration. Increasing $C R$ often results in faster convergence, while decreasing $C R$ increases search robustness [67], [70].

Most implementations of DE strategies keep the control parameters constant. Although empirical results have shown that DE convergence is relatively insensitive to different values of these parameters, performance (regarding accuracy, robustness, and speed) can be improved by finding the best values for control parameters for each new problem. 
To complement the differential mutation search strategy, DE also employs uniform crossover. Sometimes referred to as discrete recombination. In particular, DE crosses each vector with a mutant vector. The trial vector $u_{i, g}$ is developed from the elements of the target vector, $x_{i, g}$ and the elements of the donor vector, $v_{i, g}$. Then, the elements of the donor vector enter the trial vector with the crossover probability $C R$.

$$
u_{j, i, g}= \begin{cases}v_{j, i, g} & \text { if } \text { rand }_{j, i} \leq \text { CR or } j=I_{\text {rand }} \\ x_{j, i, g} & \text { if rand } \text { ran,i }_{j}>\text { CR or } j \neq I_{\text {rand }}\end{cases}
$$

The crossover probability, $C R \in[0,1]$, is a user-defined value that controls the fraction of parameter values that are copied from the mutant. To determine which source contributes a given parameter, uniform crossover compares $C R$ to the output of a uniform random number generator, $\operatorname{randj}($ ). If the random number is less than or equal to $C R$, the trial parameter is inherited from the mutant, $v_{i, g}$ otherwise, the parameter is copied from the vector, $x_{i, g}$. In addition, the trial parameter with randomly chosen index, jrand, is taken from the mutant to ensure that the trial vector does not duplicate $x_{i, g}$. Because of this additional demand, $C R$ only approximates the true probability, $p_{C R}$, that a trial parameter will be inherited from the mutant.

\subsubsection{Selection}

Selection is applied to determine which individuals will take part in the mutation operation to produce a trial vector, and to determine which of the parent or the offspring will survive to the next generation.

There are two functions for the selection operator: First it selects the individual for the mutation operation to generate the trial vector, and second, it selects the most excellent, between the parent and the offspring based on their fitness value for the next generation. In other words, If the trial vector, $u_{i, g}$, has an equal or lower objective function value than that of its target vector, $x_{i, g}$, it replaces the target vector in the next generation otherwise, the target retains its place in the population for at least one more generation (5.11). By comparing each trial vector with the target vector from which it inherits parameters, DE more tightly integrates recombination and selection than do other evolution algorithmes.

$$
x_{i, g}=\left\{\begin{array}{cr}
u_{i, g} & \text { if } f\left(u_{i, g}\right) \leq f\left(x_{i, g}\right) \\
x_{i, g} & \text { otherwise }
\end{array}\right.
$$


Once the new population is installed, repeat the process of mutation, recombination, and selection until the optimum is located, or a prespecified stopping criterion is satisfied, e.g., the number of generations reaches a preset maximum, $g_{\max }$.

in other words, to construct the population for the next generation, the deterministic selection is used: the offspring replaces the parent if the fitness of the offspring is better than its parent. Otherwise the parent survives to the next generation. This ensures that the average fitness of the population does not deteriorate.

General Differential Evolution Algorithm [66].

1) Set the generation counter, iter $=0$;

2) Initialize the mutation factor, $F$ and crossover probability $C R$;

3) Create and initialize the population $P$, based on the size and number of variables;

4) Set the boundaries of each variable $\left[x_{\min }, x_{\max }\right]$

5) while stopping condition(s) not true for each individual do, $x_{i}(g) \in P(g)$ do

6) produce the trial vector, $v_{i}(g)$ by applying the mutation operator;

7) Create an offspring, $u_{j, i},(g)$, by applying the recombination operator;

8) Evaluate the fitness, $F\left(x_{i}(g)\right)$; and

9) Evaluate the fitness, $F\left(u_{j, i},(g)\right.$;

10) Compare if $F\left(x_{i}(g)\right)$ is better than , $F\left(u_{j, i},(g)\right.$; then

11) Add $\left.x_{i}(g)\right)$ to $P(g+1)$, else,

12) Add $\left(u_{j, i},(g)\right.$ to $P(g+1)$;

13) Return the individual with the lowest fitness value as an optimal solution.

\subsection{DE operation and test}

The simplicity of DE's generate-and-test loop becomes apparent once Eqs. (5.9) to (5.11) are combined:

$$
\begin{gathered}
\mathrm{u}_{\mathrm{j}, \mathrm{i}, \mathrm{g}}=\left\{\begin{array}{cc}
\mathrm{x}_{\mathrm{r} 0, \mathrm{~g}}+\mathrm{F}\left(\mathrm{x}_{\mathrm{r} 1, \mathrm{~g}}-\mathrm{x}_{\mathrm{r} 2, \mathrm{~g}}\right) & \text { if }\left(\mathrm{rand}(0,1) \leq \mathrm{CR} \text { or } \mathrm{j}=\mathrm{j}_{\mathrm{rand}}\right) \\
\text { otherwise }
\end{array}\right. \\
x_{\mathrm{i}, \mathrm{g}} \\
x_{i, g+1}=\left\{\begin{array}{cc}
u_{i, g}, & \text { if } f\left(u_{i, g}\right) \leq f\left(x_{i, g}\right) \\
x_{i, g} & \text { otherwise }
\end{array}\right.
\end{gathered}
$$




\subsection{The flowchart of the DE algorithm}

Figure 5.4 shows a flowchart of DE. That $r 0, r 1, r 2$ and $i$ are distinct indices is not made explicit in this figure. 
1) Choose the target vector and best vector

2) Random choice of two population members

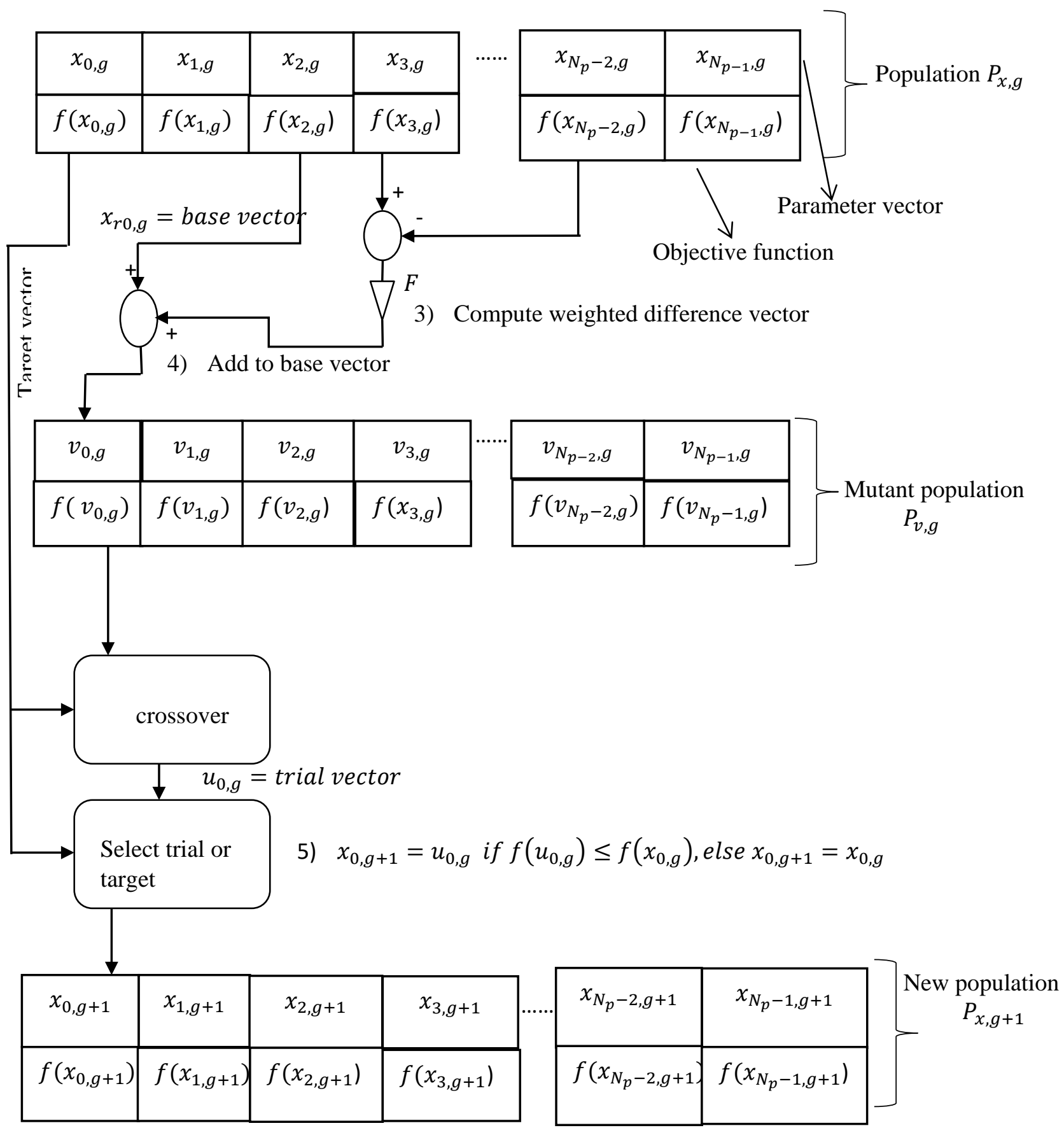

Figure 5.4 A flowchart of DE's generate-and-test loop 


\subsection{Test systems}

The proposed methodology to reconfigure the radial distribution systems and managing the grid-connected DG units is tested on two adaptive IEEE test system. Figure 5.1 shows the 16-bus test system with three initially opened tie switches. the 16-node test system is represented by its equivalent circuit in a single line diagram. The substation nodes are numbered as 1,2, and 3 respectively. Base values for this system are $12.66 \mathrm{kV}$ with 100MVA respectively. having 3 substations, three main feeders, 16 nodes, 13 sectionalizing switches, and 3 tie line switches. The system total active power is $28.7 \mathrm{MW}$, and total reactive power is 17.3MVAr. In this work, the assumption is that each one of the three transformers can carry the total loads of the network. The second example is an IEEE 33-node radial distribution system. Figure 5.5 shows the reconfiguration of the revised system with five normally opened tie switches. This test system is presented on a per phase basis and the loads along the feeder are considered as a spot load with constant P, Q loads placed at the end of the lines. In addition, each line in the system is associated with a sectionalized switch. This system has five tie-switches (dotted lines) numbered as $(33,34$, $35,36,37)$ and 32 in service branches (solid lines) and 33 nodes. The system has a total load of 3.715 MW and 2.30 MVAr.

\subsection{Simulation results and discussions}

In this dissertation, forward and backward sweep algorithm is used for load flow analysis since it is using some simple algebraic equations and gives accurate results for the distribution system. The analysis is performed in different testing criteria, for instance, both test systems are tested before and after reconfiguration without DG units, and then the test is performed with integrating single and multiple DG units. Finally, simulation and discussion using GA and proposed DE algorithm. Table 5.2 represents the load flow results for both test networks under normal condition. Distributed generators can generate or consume reactive power $Q$. Table 5.3 show the results of positive and negative output reactive power of the DG units.

Loss reduction depends on network topology and the amount, location and output power of the DG units installed in the distribution system. The integration of DG units adds positive active power injections to the grid (injects negative current). In this part, the 
installation node and capacity of DGs are not optimum. The reason is that owners of DGs determine the location and capacity of the unit to improve their economic benefits. Basically, the owners of DGs are usually individuals and non-utilities. Therefore, in this case, the size and location are not controllable. If the owner of DGs is the utility company, then the choice of the locations and sizing is important because additional DGs may cause an increase in power losses.

The proposed topology to reconfigure the distribution feeders for loss reduction and managing the grid-connected DG units are tested on two test feeders 16-bus test system shown in figure 5.1, and IEEE 33-bus test system shown in figure 5.5. The test results are obtained as follows:

1) distribution feeder results before reconfiguration,

2) distribution feeder results after reconfiguration,

3) distribution feeder results after reconfiguration with $\mathrm{DG}$,

4) distribution feeder results after reconfiguration with DG using a genetic algorithm,

5) distribution feeder results after reconfiguration with DG and using the proposed DE algorithm, and comparing the obtained results with the ones obtained using GA. 


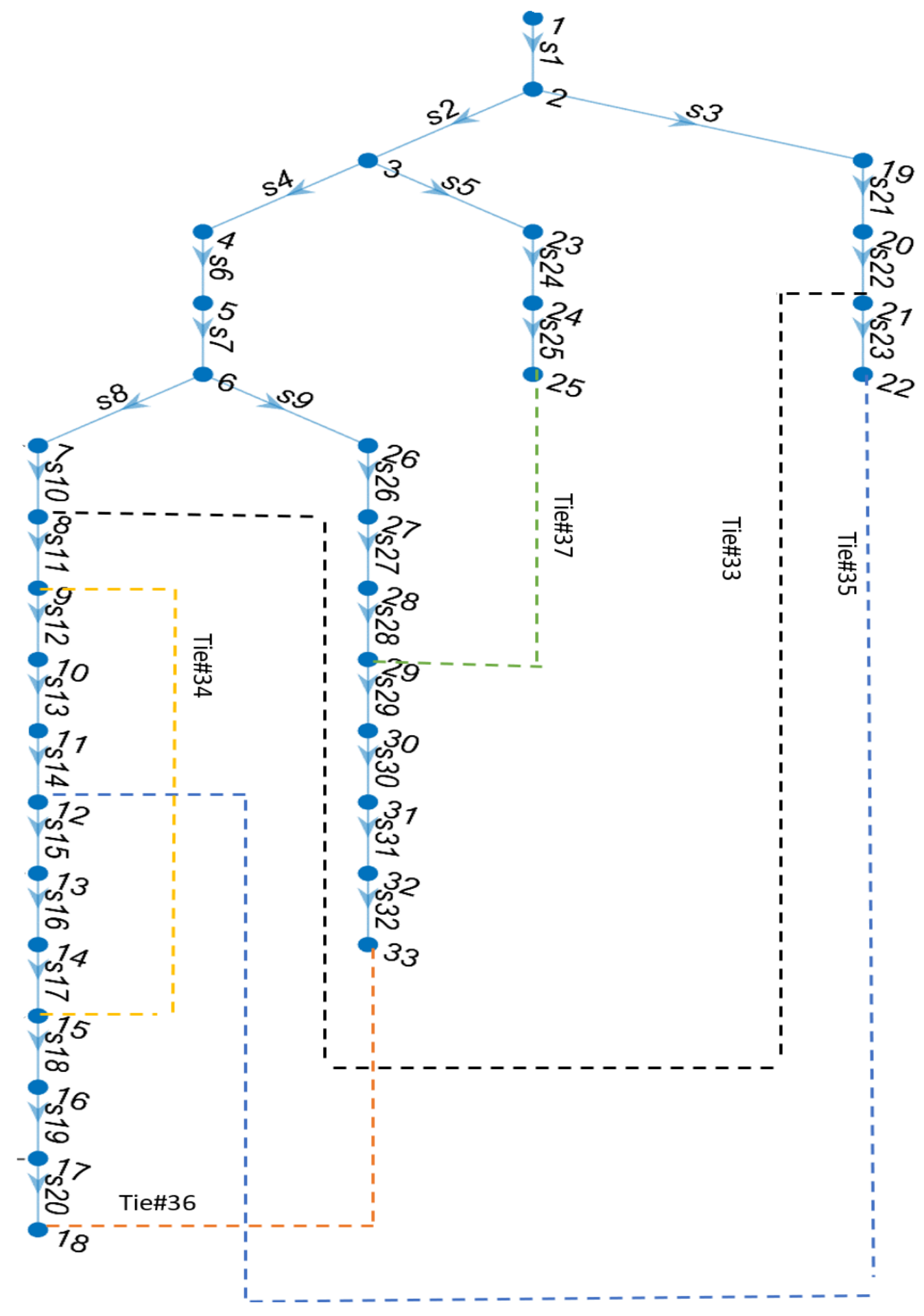

Figure 5.5 Single line diagram of IEEE 33 bus distribution system, s denoted switch and the dotted lines represent the tie switches 
The simulation parameters of the proposed DE algorithm are shown in Table 5.1.the most important ones are the mutation and crossover factors because they create a new trial matrix from mutation and recombination procedure. then each trial in been evaluated and the fitness results are compared with the original population matrix in order to obtain the best fitness and memorize it.

Table 5-1 Simulation parameters of DE

\begin{tabular}{|l|l|l|l|}
\hline $\begin{array}{l}\text { Population } \\
\text { size }\end{array}$ & $\begin{array}{l}\text { Mutation } \\
\text { factor }\end{array}$ & $\begin{array}{l}\text { Crossover } \\
\text { rate }\end{array}$ & Max iteration \\
\hline 100 & 0.5 & 0.8 & 50 \\
\hline
\end{tabular}

Figure 5.6 shows the performance of the DE algorithm such that the system reached the optimal minima in a faster time and less iteration. The idea herein is that for each evaluation step the size of the population matrix is updated in order to prevent from evaluating the same trail more than ones. This means that, since the topology is to memorize the trials with the best fitness value, the stopping criteria is met when the rows of the trial matrix become the same. In contrast with GA, the minimum loss reduction found by GA is 0.0061 p.u, but the ones obtained by DE was 0.0060 this because DE screech from a larger space and moreover the mutation operation is also expanding the search area.

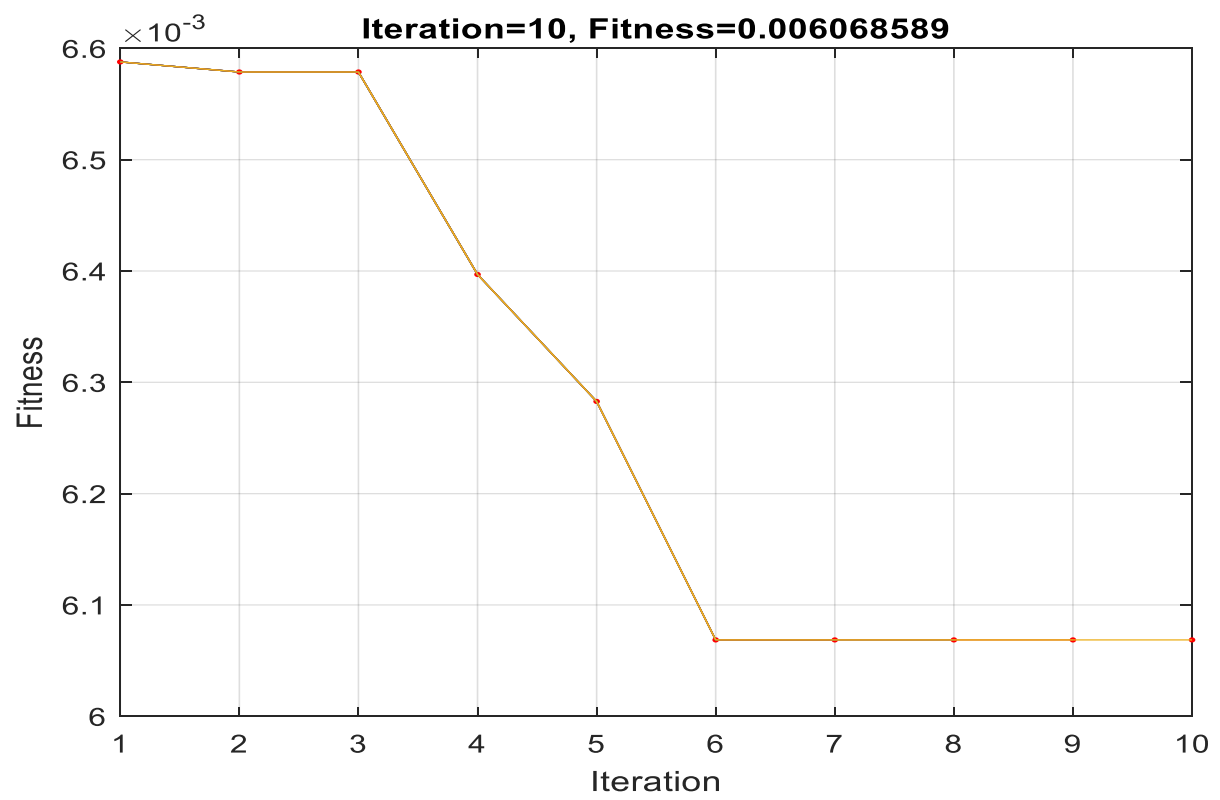

Figure 5.6 Convergence curve of the best fitness value of DE algorithm 
Table 5-2 Simulation Results of Both Test Systems Before and After Feeder Reconfiguration and after reconfiguration using GA and proposed DE

\begin{tabular}{|c|l|l|l|l|l|l|}
\hline \multicolumn{2}{|l|}{} & $\begin{array}{l}\text { System } \\
\text { Real } \\
\text { Case study }\end{array}$ & $\begin{array}{l}\text { System } \\
\text { Reactive } \\
\text { Power } \\
\text { Loss } \\
\text { (p.u) }\end{array}$ & $\begin{array}{l}\text { Minimum } \\
\text { system } \\
\text { (p.u) }\end{array}$ & $\begin{array}{l}\text { Current } \\
\text { from } \\
\text { substation } \\
\text { voltage } \\
\text { (p. u) }\end{array}$ & Tie-switch \\
opened
\end{tabular}


Table 5-3 Simulation Results of Both Test Systems After Feeder Reconfiguration Using Proposed DE

\begin{tabular}{|c|c|c|c|c|c|c|c|}
\hline \multirow{2}{*}{\multicolumn{2}{|c|}{$\begin{array}{l}\text { DG unit } \\
\text { location }\end{array}$}} & \multicolumn{2}{|l|}{ DG Size } & \multirow{2}{*}{$\begin{array}{l}\text { System } \\
\text { Real } \\
\text { Power } \\
\text { Loss } \\
\text { (p.u) }\end{array}$} & \multirow{2}{*}{$\begin{array}{l}\text { System } \\
\text { Reactive } \\
\text { Power } \\
\text { Loss } \\
\text { (p.u) }\end{array}$} & \multirow{2}{*}{$\begin{array}{l}\text { Current } \\
\text { from } \\
\text { substation } \\
\text { (p.u) }\end{array}$} & \multirow[b]{2}{*}{$\begin{array}{l}\text { Tie-switch } \\
\text { opened }\end{array}$} \\
\hline & & $\mathrm{P}_{\mathrm{DG} \_}$output ( & $\mathrm{Q}_{\mathrm{DG}-}$ output(r & & & & \\
\hline \multirow{6}{*}{ 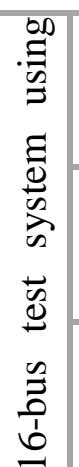 } & \multirow{2}{*}{12} & \multirow{2}{*}{0.0600} & 0.0360 & 0.0029 & 0.0032 & 0.2738 & $12,21,26$ \\
\hline & & & -0.0360 & 0.0037 & 0.0040 & 0.3265 & $12,22,14$ \\
\hline & \multirow{2}{*}{10} & \multirow{2}{*}{0.0300} & 0.0180 & 0.0041 & 0.0046 & 0.3135 & $15,21,14$ \\
\hline & & & -0.0180 & 0.0042 & 0.0047 & 0.3470 & $12,21,26$ \\
\hline & \multirow{2}{*}{16} & \multirow{2}{*}{0.0360} & 0.0240 & 0.0011 & 0.0016 & 0.3129 & $12,21,26$ \\
\hline & & & -0.0240 & 0.0014 & 0.0021 & 0.4075 & $12,22,14$ \\
\hline \multirow{6}{*}{ 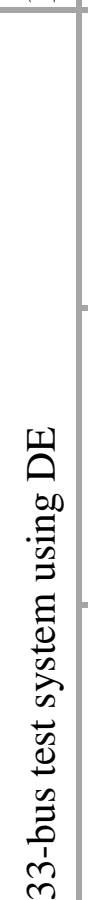 } & \multirow{2}{*}{30} & \multirow{2}{*}{0.0120} & 0.0030 & 0.0008 & 0.0006 & 0.0584 & $\begin{array}{l}8,27,33,34 \\
, 36\end{array}$ \\
\hline & & & -0.0030 & 0.0009 & 0.0007 & 0.0661 & $\begin{array}{l}8,27,33,34 \\
, 36\end{array}$ \\
\hline & \multirow{2}{*}{$\begin{array}{l}16,2 \\
2\end{array}$} & \multirow{2}{*}{$\begin{array}{l}0.0060 \\
0.0075\end{array}$} & $\begin{array}{l}0.0023 \\
0.0015\end{array}$ & 0.0013 & 0.0009 & 0.0557 & $\begin{array}{l}8,27,33,34 \\
, 36\end{array}$ \\
\hline & & & $\begin{array}{ll}-0.0023, & - \\
0.0015 & \end{array}$ & 0.0012 & 0.0008 & 0.0650 & $\begin{array}{l}8,27,33,34 \\
, 36\end{array}$ \\
\hline & \multirow{2}{*}{$\begin{array}{l}18,3 \\
3\end{array}$} & \multirow{2}{*}{$\begin{array}{l}0.0105,0.01 \\
20\end{array}$} & $\begin{array}{l}0.0015,0.001 \\
2\end{array}$ & 0.0013 & 0.0011 & 0.0477 & $\begin{array}{l}8,27,33,34 \\
, 36\end{array}$ \\
\hline & & & $\begin{array}{l}-0.0015, \quad- \\
0.0012\end{array}$ & 0.0012 & 0.0009 & 0.0718 & $\begin{array}{l}8,27,33,34 \\
, 36\end{array}$ \\
\hline
\end{tabular}

The simulation results of the first case for the network reconfiguration without placing DG units are shown in Table 5.2. It is evinced that by applying the proposed method the system real/reactive power losses are minimized to 0.0060 and 0.0064 p.u respectively for the 16bus test system, and 0.0010 and 0.0008 p.u for the 33-bus system. The weakest node 
voltage magnitude is improved to 0.9588 p.u and 0.9382 p.u for both test system respectively. Table 5.3 shows the results for the second case after installing a single and multiple DGs at different locations using DE algorithm. The obtained results show that system performance in terms of power losses and voltage profile has been improved more than those shown in Table 5.2.

\subsection{Conclusion}

In this chapter, a new approach has been proposed to reconfigure the distribution network with the impact of the existent and planned DG units. In addition, different loss reduction and load balancing scenarios, such as before/after network reconfiguration, with/without including DGs, and after reconfiguration with the presence of single and multiple DG units are also simulated to validate the effectiveness of the proposed method. An efficient differential evolution algorithm is used in the optimization process of the network reconfiguration. The proposed methods are tested on 16- and 33-bus systems. The simulation results have demonstrated that the DE algorithm achieved better performance than traditional methods including genetic algorithm. 


\section{Chapter 6}

\section{Optimal Placement and Sizing of Distributed Generation in a Radial Distribution System Using Differential Evolution Algorithm (DEA)}

\subsection{Introduction}

The continuous growth in power demand, lack in active power generation as well as limitations of traditional transmission and distribution (T\&D) infrastructure have led to increased interest in distributed generation (DG) utilization. In addition, DG which tend to utilize renewable energy, can reduce greenhouse emissions and reduce the demanded power that must be produced and provided by incorporated power plants. Optimal placement and sizing of DG units can reduce the power losses of distribution networks, release power capacity for existing or expanding loads, and improve voltage profile at the load nodes. This chapter proposes an efficient optimization technique based on Differential Evolution Algorithm (DEA) for optimal allocation and sizing of DGs units in distribution systems. The algorithm is based on the node voltage sensitivities index (VSI) in which the node, that is most sensitive to voltage collapse is identified. Additionally, the algorithm is also based on the real power losses of each branch with respect to real power injection at each node. The weakest bus is considered to be the one with maximum active power loss. System power loss data can be collected based in a certain time period, it could be seasonal or yearly data collection. In this work, both the real output power of the DGs and reactive output power of the DGs inverter has been considered.

Due to the environmental impact concerns and incentives from regulators, Distributed Generation (DG) has become the central part of the distribution networks. Distributed generation is known as an on-site generation or decentralized generation. It referred to the generation of electricity for use on-site, rather than transmitting energy over the electric grid from a large, centralized facility. That is to take its advantages of cleaner energy, less loss, and local power supply. The impact of dispersed generation (DG) by adding an active power source to the distribution network is aiming mainly to reduce the active power loss in the distribution network and as a result, improving the voltage profile at the network. Nowadays, these renewable DG are required to equip with reactive power devices (such as 
static VAR compensators, capacitor banks, etc.), to provide reactive power as well as to control the voltage at their terminal bus. DGs have various technical benefits such as voltage profile improvement, relief in feeder loading, power loss minimization, stability improvement, and voltage deviation mitigation. In other words, the distributed generation does not benefit the network if placed in any random location in the system. Therefore, it is necessary to determine the optimal location and size of the DG. In fact, most distribution networks are weak and radial in nature with low short-circuit capacity. Therefore, there is a limit to which power can be injected into the distribution network without compromising the power quality and the system stability. Thus, this work is aiming to investigate this by connecting DG technologies to the grid and demonstrating the system voltage within a defined boundary $[0.95-1.05$ p. u].

The IEEE Standard 1547 has to be obeyed for the interconnection of Distributed Energy Resource (DER). DER include distributed generators and energy storage systems. The standards provide requirements of connecting the DGs to the grid, relevant to the performance, operation, safety considerations, and maintenance of the interconnection. Such requirements are that, first, the DER units do not unintentionally provide power to the adjacent electricity customers or to the utility grid when the grid has lost its power supply from the transmission system [72]. Second, in the case of an open-phase condition, the DGs must detect and stop injecting power to the grid. Third, in the case of voltage regulation requires the operator of the distribution grid and the DGs are required to coordinate with each other to allow the DG to participate by changing its real and reactive power outputs.

To study the impact of integrating DGs units into the distribution network, several kinds of literature have proposed the use of different optimization techniques. The optimum placement and sizing are done to achieve different objectives. In [73] evolutionary programming with the objective of maximizing the reduction of the load supply costs was used. Besides, Siano [74] proposed the combination of a Genetic Algorithm (GA) and Optimal Power Flow (OPF) to efficiently site and size a predefined number of DGs. It differs from other proposed methods that only define the optimal locations and capacities of DG as a means of ensuring that the maximum amount of DG can be connected to the existing and future networks. Also, in [75] Particles Swarm Optimization (PSO) was used 
for optimal placement of multi-DGs with the aim of minimizing the total real power loss. Other various methods such as simulated annealing technique [76], Tabu search method [77] [78], and artificial bee colony (ABC) [79], etc. Alarcon- Rodriguez et al. [80]introduced the use of time-varying loads for analysis of reliability and efficiency of distribution networks with DG. In [81], Ochoa et al. proposed a time-varying approach in demand load and generation, and steady-state analysis of technical issues such as losses, voltages, reserve capacity of conductors, and short-circuit levels was presented.

This work analyzes the impact of the DG installation on the performance of the distribution network and its parameters such as voltage, active and reactive power loss [82]. The topology is that the DG unit is injecting real power to the selected node and meanwhile, the voltage of all nodes is checked as well as the branch currents to prevent from having overvoltage or exceeding the line capacity limits. The feasible solution is the case of satisfying all the operation constraints, such as node voltages has to be within sitting limits, brunch currents also within its maximum and minimum limits, the size of the DG is specified. The solution methodology herein is to evaluate the system whit out DG connection and then reconfigure the system by closing the tie switches and specify the tie with the maximum voltage difference and consider closing this tie first, then the DG location is obtained using the sensitivity of power losses with respect to real power injection at each bus. Then the most sensitive bus is selected for installing the DG unit. Because the integration of the DG adds positive real power injections, the optimal location is the one with the most negative sensitivity in order to get the largest power loss reduction. Finally, after the location is specified the proposed DEA is used to obtain the optimal size of the DG unit. Only the feasible solution who satisfy all the constraint is considered.

This chapter proposed a Differential Evolution Algorithm for the sizing of the DG unit in a distribution network the technique will minimize real power losses and improve system voltage profile. The optimizations technique are performed on three stages, first, the network reconfiguration is performed, then, the optimal location of the planned DG is determined, and finally, the size of DG located at its optimal location is obtained by using the proposed DEA. In this chapter, the proposed DEA is applied on a revised version of the IEEE 33-bus test system. Differential evolution (DE) is a stochastic, population-based search strategy developed by Storn and Price [83] in 1995. While DE shares similarities 
with other evolutionary algorithms (EA), it differs significantly in the sense that information from the current population is used to guide the search process. In DE algorithm, all solutions have an equal opportunity of being preferred as parents, and selection does not depend on their fitness values. In DE, each new solution fashioned competes with its parent and the superior one wins the contest [84]. In DE operation the child vector is generated by applying the mutation and crossover operation. In mutation operation, a trial vector is generated with the help of the objective vector and two erratically preferred individuals. The deviation in the objective vector depends on mutation factor $\mathrm{F}$ and the difference between the randomly selected individuals. On the other hand, the crossover operation is applied between the objective vector and parent vector to generate the child vector using the crossover probability (CR). Like any other method, these tuning factors have a boundary such that large values may result in skipping of actual solutions, and small values also may cause performance degrades. The conflicts in the child vector from the parent vector depends on the values of $\boldsymbol{F}$ and $\boldsymbol{C R}$.

This chapter is organized as follows: section 6.2 represents the problem formulation of power loss minimization. Section 6.3 Presents the proposed Differential Evolution algorithm DEA. Section 6.4 provides the approach to find the optimal placement and size of DG units. Simulation results and discussion are given in section 6.5. Finally, Conclusions are provided in section 6.6.

\subsection{Problem formulation}

The problem considered herein is to minimize the system power losses and to improve the voltage profile. The integration of single or multiple DGs units in the power distribution network is a challenging problem that involves complex nonlinear equations. Sizing and allocation of the DGs is a vital parameter as the random placement of DG in the grid can cause a negative consequence such as an increase in system total power losses and out-oflimit voltages. On the contrary, if the DGs are precisely placed and optimally sized, then it will improve the system's overall efficiency. This work mainly focuses on identifying the optimal size and location of installing DGs units in the grid in the way all the constraints are satisfied. The problem is formulated as a multi-objective problem, whose objectives include minimization of total system active and reactive power losses, minimization of 
feeder's currents imbalance, and minimization of node voltage deviation considering the substation as a reference.

The loss minimization is formulated as:

$$
\operatorname{minP}_{\text {loss }}(\mathrm{x})
$$

with

$$
\mathrm{P}_{\text {loss }}=\sum_{\mathrm{i}=1}^{\mathrm{Nb}} \mathrm{R}_{\mathrm{i}} *\left|\mathrm{I}_{\mathrm{i}}\right|^{2}
$$

such that,

$$
\begin{array}{ll}
I_{i} \leq I_{\max } & \forall i=1,2 \ldots, N b \\
V_{\text {min }} \leq V_{j} \leq V_{\text {max }} & \forall j=1,2, \ldots, N
\end{array}
$$

where, $P_{\text {loss }}=$ total line losses of the feeder

$x=$ status vector of the switch

$N b=$ total number of branches in the whole system

$I_{i}=$ current magnitude of branch $i$

$I_{\max }=$ upper limit of branch current magnitude

$V_{j}=$ voltage magnitude of branch $j$

$V_{\min }, V_{\max }=$ lower and upper limit of node voltage magnitude, herein, $0.95 \leq V_{j} \leq 1.05$

$R_{i}=$ resistance of branch $i$

$N=$ total number of buses

$x=\left[s w_{1} s w_{2} s w_{3}, \ldots s w_{\text {number of vairables }]}\right.$

The total power loss of any branch is determined by summing up all the losses of all branch sections beyond this particular branch of the feeder, that is:

$$
\begin{aligned}
\text { Delta }_{\mathrm{P}} & =\sum_{\mathrm{branch}=1}^{\mathrm{Nb}} \mathrm{I}_{\mathrm{branch}}{ }^{2} * \mathrm{R}_{\text {branch }} \\
\text { Delta }_{\mathrm{Q}} & =\sum_{\mathrm{branch}=1}^{\mathrm{Nb}} \mathrm{I}_{\mathrm{branch}^{2}}{ }^{2} * \mathrm{X}_{\mathrm{branch}}
\end{aligned}
$$


where,

$\operatorname{Delta}_{P}=$ real power loss

$\operatorname{Delta}_{Q}=$ reactive power loss.

The operating constraints of the system are defined as inequality and equality constraints.

\subsubsection{Inequality Constraints}

\subsubsection{Power generation limits}

The output generating power of the DGs unit must be within a limit.

Thus,

$$
\begin{aligned}
& P_{D G i, \min } \leq P_{D G i} \leq P_{D G i, \max } \\
& Q_{D G i, \min } \leq Q_{D G i} \leq Q_{D G i, \max }
\end{aligned}
$$

In this chapter, the feasible solution is obtained in such a way the optimal location and size of the connected DG satisfies all the operating constraints. Considering that all the node voltages are within limits and that there are no violations in the value of voltage.

6.2.1.2 Branch power limit constraint:

$$
\begin{gathered}
P_{j}^{2}+Q_{j}^{2} \leq\left(S_{j}^{\max }\right) \\
P_{D G i}^{\min } \leq P_{D G i} \leq P_{D G i}^{\max } \\
Q_{D G i}^{\min } \leq Q_{D G i} \leq Q_{D G i}^{\max }
\end{gathered}
$$

6.2.1.3 Bus voltage limits

$$
V_{\min } \leq V_{i} \leq V_{\max }
$$

6.2.1.4 Branch current magnitude: maximum limit

$$
\left|I_{i}\right| \leq I_{\max }
$$

\subsubsection{Equality Constraints}

Power balance: the power flow equations must be satisfied

$$
\begin{aligned}
& P_{i}=P_{D G i}-P_{D i} \\
& Q_{i}=Q_{D G i}-Q_{D i}
\end{aligned}
$$


where,

$P_{D G i}$ and $Q_{D G i}$ are distributed power generation at bus $i . P_{D i}$ and $Q_{D i}$ are the loads at bus $i$.

In this dissertation, the feasible solution is obtained in such a way that optimal location and size of the connected DG satisfies the operating constraints, by taking into account that all the node voltages are within limits and that there are no violations in the value of voltage.

\subsection{Differential Evolution Algorithm}

Differential Evolution is a Global Optimization algorithm searching from an ample space of candidate solutions. DE algorithm is used for multidimensional real-valued functions, so DE does not need the optimization problem to be differentiable as is mandatory for traditional optimization methods [84]. Differential Evolution algorithm involves three unique advantage, does not depend on the initial population values in finding the global minima, using few parameters, and fast convergence. DE differs from Genetic Algorithm in such a way that it relies on mutation scheme while GA relies on crossover processer. Also, DE uses a non-uniform crossover, it can take a child vector parameter from one parent more often than it does from others. The DE algorithm also uses the mutation mechanism and selection operation to point the search toward the prospective region in the search area. the

DE algorithm optimizes a problem by considering a population of candidate solutions and generating new contestant solutions by combining existing ones according to its straightforward formulae, and then memorizing whichever candidate solution has the excellent score or fitness on the optimization problem at hand.

General problem formulation of DE is as follows

DE differs from other algorithms such that:

1) Mutation is applied first to generate a trial vector, which is then used within the crossover operator to produce one offspring.

2) Mutation step sizes are not sampled from a prior known probability distribution function. 
3) In DE, mutation step sizes are influenced by differences between individuals of the current population.

For an objective function $f: X \subseteq \mathbb{R}^{D} \rightarrow \mathbb{R}$ where the feasible region $X \neq \emptyset$, the minimization problem is to find $x^{*} \in X$ such that

$$
\mathrm{f}\left(\mathrm{x}^{*}\right) \leq \mathrm{f}(\mathrm{x}) \forall \mathrm{x} \in \mathrm{X}
$$

such that:

$$
f\left(x^{*}\right) \neq-\infty
$$

The parameter vectors of the optimization function have the form:

$$
\mathrm{x}_{\mathrm{i}, \mathrm{G}}=\left[\mathrm{x}_{1, \mathrm{i}, \mathrm{G}}, \mathrm{x}_{2, \mathrm{i}, \mathrm{G}}, \ldots \ldots \mathrm{x}_{\mathrm{D}, \mathrm{i}, \mathrm{G}}\right] \forall \mathrm{i}=1,2 \ldots, \mathrm{N}
$$

where,

$N=$ population size

$G=$ generation number

$D=$ D-dimensional search space

\subsubsection{Initialization}

The initialization procedure is to define the upper and lower bounds for each parameter such that

$$
x_{j}^{L} \leq x_{j, i, 1} \leq x_{j}^{U}
$$

In distribution systems especially in this part, we are dealing with a switch (e.g., integer parameters), in this case sitting the upper and lower boundaries of each variable must obey these rules:

1. There should be no common switches between two meshes.

2. Only one open switch in each mesh.

3. Switches of the end node loads remain on and should not be considered in the boundary.

4. The radial structure must be retained.

Then randomly select the initial parameters values uniformly on the intervals $\left[x_{j}^{L}, x_{j}^{U}\right]$. 
The population matrix is formulated based on the number of variables (e.g., number of open ties) and maximum population size.

$$
\operatorname{pop}_{\text {matrix }}=\left[\begin{array}{cc}
\mathrm{x}_{1} & \mathrm{x}_{2}, \ldots \ldots \mathrm{x}_{\mathrm{n}} \\
& \vdots \\
& \vdots \\
\mathrm{x}_{1 \mathrm{p}} & \mathrm{x}_{2 \mathrm{p}} \ldots \ldots \mathrm{x}_{\mathrm{np}}
\end{array}\right]
$$

Where the $n$ denoted number of variables, and $p$ is the population size.

\subsubsection{Statistics}

Load-flow computation (6.1) is used as a fitness function by adding some constraints such as:

$$
\mathrm{F}=\mathrm{P}_{\text {loss }}\left(\mathrm{S}_{\mathrm{V}}\right)+\sum_{\mathrm{i}=1}^{\mathrm{Nb}} \lambda_{\mathrm{I}, \mathrm{i}} *\left(\mathrm{I}_{\mathrm{i}}-\mathrm{I}_{\mathrm{lim}}\right)^{2}+\sum_{\mathrm{j}=1}^{\mathrm{N}} \lambda_{\mathrm{V}, \mathrm{j}} *\left(\mathrm{~V}_{\mathrm{j}}-\mathrm{V}_{\mathrm{lim}}\right)^{2}
$$

where, $\lambda_{I, i}$, and $\lambda_{V, j}$ are the penalty factors that can be adjusted in the optimisation procedure. $I_{\text {lim }}$ and $V_{\text {lim }}$ are defined as:

$$
\begin{gathered}
I_{\lim }= \begin{cases}I_{i} & \text { if } I_{i} \leq I_{\max } \\
I_{\max } & \text { if } I_{i}>I_{\max }\end{cases} \\
V_{\lim }= \begin{cases}V_{j} & \text { if } V_{\min } \leq V_{j} \leq V_{\max } \\
V_{\min } & \text { if } V_{j}<V_{\max } \\
V_{\max } & \text { if } V_{j}>V_{\max }\end{cases}
\end{gathered}
$$

\subsubsection{Mutation}

One of the DE advantages is searching from a large search space, and the mutation procedure expands the search space. Each of the $\mathrm{N}$ parameter vectors undergoes mutation, recombination, and selection. To illustrate that, for a given parameter vector $x_{i, G}$ randomly select three vectors $x_{r 1, G}, x_{r 2, G}$ and $x_{r 3, G}$ such that the indices $i, r 1, r 2$ and $r 3$ are distinct. Then calculate the donor vector by adding the weighted difference of two of the vectors to the third such that:

$$
\mathrm{v}_{\mathrm{i}, \mathrm{G}+1}=\mathrm{x}_{\mathrm{r} 1, \mathrm{G}}+\mathrm{F}\left(\mathrm{x}_{\mathrm{r} 2, \mathrm{G}}-\mathrm{x}_{\mathrm{r} 3, \mathrm{G}}\right)
$$


where, $\mathrm{F}=$ mutation scale factor is a constant from $[0,1]$.

\subsubsection{Recombination}

Recombination incorporates successful solutions from the previous generation. The trial vector $u_{i, G+1}$ is developed from the elements of the target vector, $x_{i, G}$ and the elements of the donor vector, $v_{i, G+1}$. Then, the elements of the donor vector enter the trial vector with the probability CR.

Thus,

$$
\begin{gathered}
\mathrm{u}_{\mathrm{j}, \mathrm{i}, \mathrm{G}+1}=\left\{\begin{array}{l}
\mathrm{v}_{\mathrm{j}, \mathrm{i}, \mathrm{G}+1} \text { if } \operatorname{rand}_{\mathrm{j}, \mathrm{i}} \leq \mathrm{CR} \text { or } \mathrm{j}=\mathrm{I}_{\text {rand }} \\
\mathrm{x}_{\mathrm{j}, \mathrm{i}, \mathrm{G}} \text { if rand } \mathrm{ran}_{\mathrm{j}, \mathrm{i}}>\mathrm{CR} \text { or } \mathrm{j} \neq \mathrm{I}_{\text {rand }}
\end{array}\right. \\
i=1,2, \ldots, N ; j=1,2, \ldots, D
\end{gathered}
$$

where,

rand $j, i \sim U[0,1], I_{\text {rand }}$ is a random integer from $[1,2 \ldots, \mathrm{D}]$, and $I_{\text {rand }}$ ensures that $v_{i, G+1} \neq x_{i, G}$

\subsubsection{Selection}

There are two functions for the selection operator. First, it selects the individual for the mutation operation to generate the trial vector. Second, it selects the most excellent for the parent and the offspring based on their fitness value for the next generation. The target vector $x_{i, G}$ is compared with the trial vector $v_{i, G+1}$ and the one with the lowest function value is admitted to the next generation.

$$
\mathrm{x}_{\mathrm{i}, \mathrm{G}+1}= \begin{cases}\mathrm{u}_{\mathrm{i}, \mathrm{G}+1} \text { if } \mathrm{f}\left(\mathrm{u}_{\mathrm{i}, \mathrm{G}+1}\right) \leq \mathrm{f}\left(\mathrm{x}_{\mathrm{i}}, \mathrm{G}\right) \\ \mathrm{x}_{\mathrm{i}}, \mathrm{G} & \text { otherwise }\end{cases}
$$

Finally, mutation, recombination a, $\mathrm{d}$ selection continues until some stopping criterion is met.

The steps of the general Differential Evolution Algorithm are shown as follows: [85]

14) Set the generation counter, iter $=0$;

15) Initialize the mutation factor, $F$ and crossover probability $C R$;

16) Create and initialize the population $P$, based on the size and number of variables; 
17) Set the boundaries of each variable $\left[x_{\min }, x_{\max }\right]$

18) while stopping condition(s) not true do the following for each individual, $x_{i}(G) \in$ $P(G)$ do

19) Create the trial vector, $v_{i,}(G)$ by applying the mutation operator;

20) Create an offspring, $u_{j, i},(G)$, by applying the recombination operator;

21) Evaluate the fitness, $F\left(x_{i}(G)\right)$; and

22) Evaluate the fitness, $F\left(u_{j, i},(G)\right.$;

23) Compare if $F\left(x_{i}(G)\right)$ is better than, $F\left(u_{j, i},(G)\right.$; then

24) Add $\left.x_{i}(G)\right)$ to $P(G+1)$, else,

$25)$ Add $\left(u_{j, i}(G)\right.$ to $P(G+1)$;

26) Return the individual with the best fitness as an optimal solution. 


\subsection{DEA flowchart}

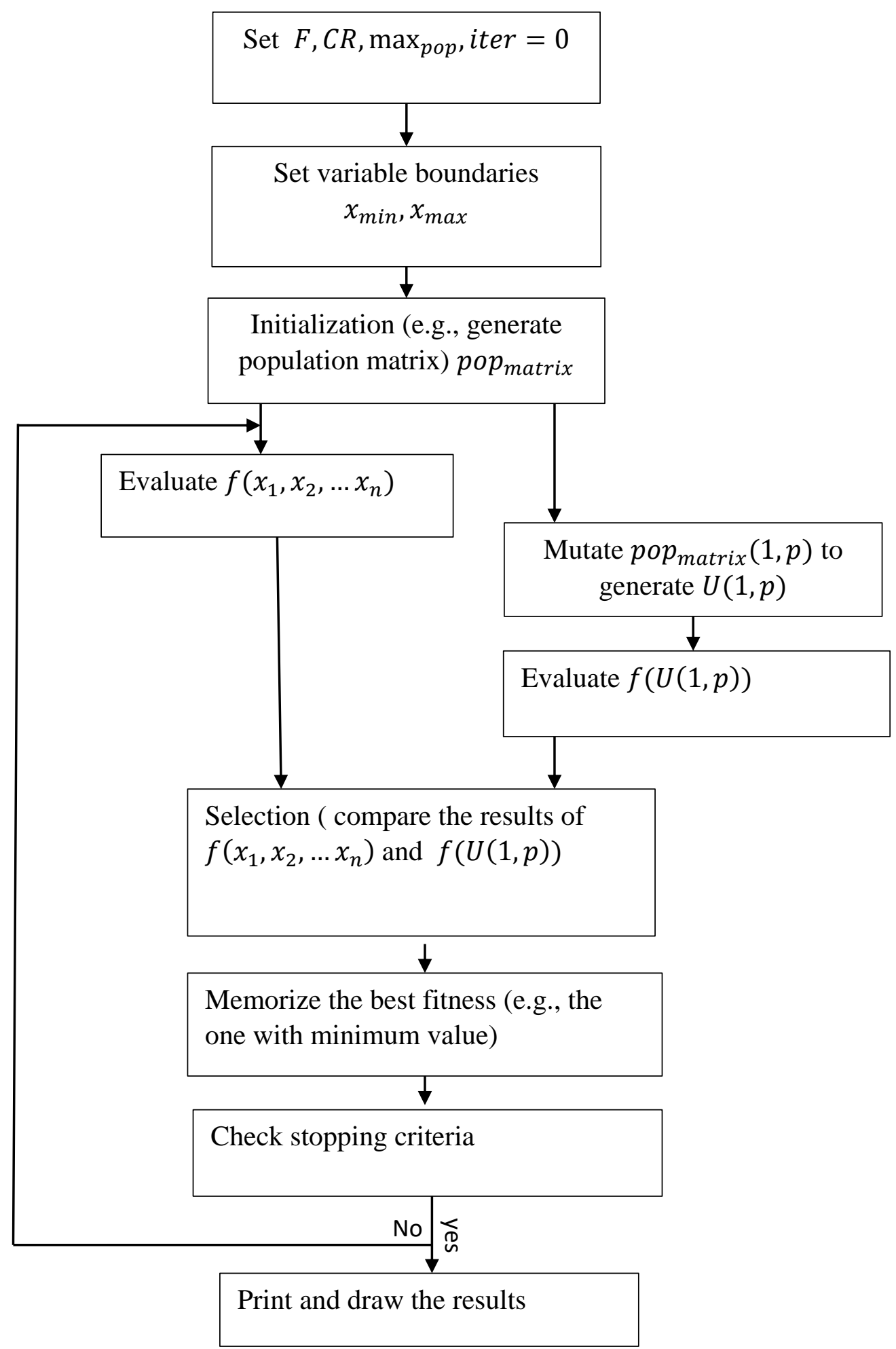

Figure 6.1 DEA flowchart 


\subsection{Optimum placement and sizing of DG units}

Power loss reduction problems are depending on the network topology and the number of connected DG, location, the output power of the DGs units installed in the grid. The connected DGs must obey the IEEE Standard 1547. Also, it can be aggregated into an equivalent unit.

\subsubsection{Optimal Location of DG units}

The DG location is obtained using the sensitivity of power losses with respect to real power injection at each bus. The most sensitive bus is selected for installing the DG unit. Because the integration of the DG adds positive real power injections, the bus with the highest negative sensitivity value is considered as the weakest bus in the system, and DG unit will be placed at that respective bus. The mathematical formulation considered in the optimal placement is the maximum of (6.3), more details can be found at [86] [87], and is given as:

$$
\mathrm{DG}_{\text {location }}=\max \left(\operatorname{Delta}_{\mathrm{P}}(\mathrm{Nb})\right)
$$

The planned DG unit is modeled as a positive current injection located at its obtained optimal location.

$$
I_{D G, i}=\frac{P_{D G, i}-j Q_{D G, i}}{V_{i}^{*}}
$$

It has been assumed that the study is implemented on a single line test system, but in a real three-phase network, the injected current from DG unit still can be computed using (6.16) by adding $\mathrm{B}$ and $\mathrm{C}$ phases. Now, in the case of three phase system, the type of the DG unit will be matter and has to be carefully considered. If the DG unit is considered or operates at a constant voltage, then two loop computation [88] is needed to get the equivalent current injection. The inner loop calculates the reactive power output of the DG unit which is necessary to keep the node voltage magnitude at the specified limits in an iterative manner, and the outer loop computes the current injection with the initially specified active and reactive power calculated from the inner loop, However, if the DG unit operates at constant power then the equivalent current injection could be directly obtained using (6.16) because the active and reactive power values are provided. In addition to the three-phase networks it is known that although a single-phase or two-phase branches usually exist in the 
unbalanced networks [89], in this particular case it's still valid such that the values of the missing corresponding phase impedances are set to zero, and as a result the currents and voltages of the missing phases are detected from the results, and keeping in mind zero phase impedance are only for the computation purpose and it does not indicate short circuit condition.

\subsubsection{The optimum size of DG units}

The optimum sizes of the DG units to be installed at the obtained optimal location is determined to minimize system total power losses and improve the voltage profile. Thus, for a reconfigurable system with connected DG units. The solution strategy is to generate a set of random DG sizes within a specified range using the proposed DEA. Placed at the obtained location (6.15) and then power flow is executed for each DG size. The fitness function is evaluated for total power reduction, and the feasible solution is obtained.

The steps of network reconfiguration, and finding the location and the size of the DGs units using the DEA are explained as follows:

Step1_ Read system data.

Step2_ Run power flow without DG connection.

Step3_determine the DG location using (6.15)

Step4_by using DEA do the following:

1) reconfigure the system following our method in [82]

2) generate a random size of DG and evaluate the fitness function for each size, if all the constraints are satisfied and all the node voltages are between the minimum and maximum boundaries, write down the optimal solution and do the same for another switch statues.

Step5_ after evaluating all the chromosomes of the population matrix and after applying the operating parameters like mutation, crossover, and recombination, note down the optimal solution with a minimum power loss and an accepted voltage.

Step6_ note down the real and reactive power loss and voltage magnitude. 


\subsection{Simulation results and discussions}

The effectiveness of the proposed DEA to solve the optimal size and location of the DGs units followed by the network reconfiguration is illustrated using the IEEE 33-bus radial distribution test feeder. The simulation results have produced the desired results.

Figure 6.2 presents the test system of a 33-node radial distribution system [90]. This test system is presented on a per phase basis, and the loads along the feeder are considered as a spot load with constant $P, Q$ loads placed at the end of the lines. In addition, each line in the system is associated with a sectionalized switch. This system has five tie-switches (dotted lines) numbered as $(33,34,35,36,37)$ and 32 in service branches (solid lines) and 33 nodes. The system has a total load of 3.715 MW and 2.30 MVAR without any connected DG unit. Base values are $12.66 \mathrm{kV}$ and 100MVA respectively.

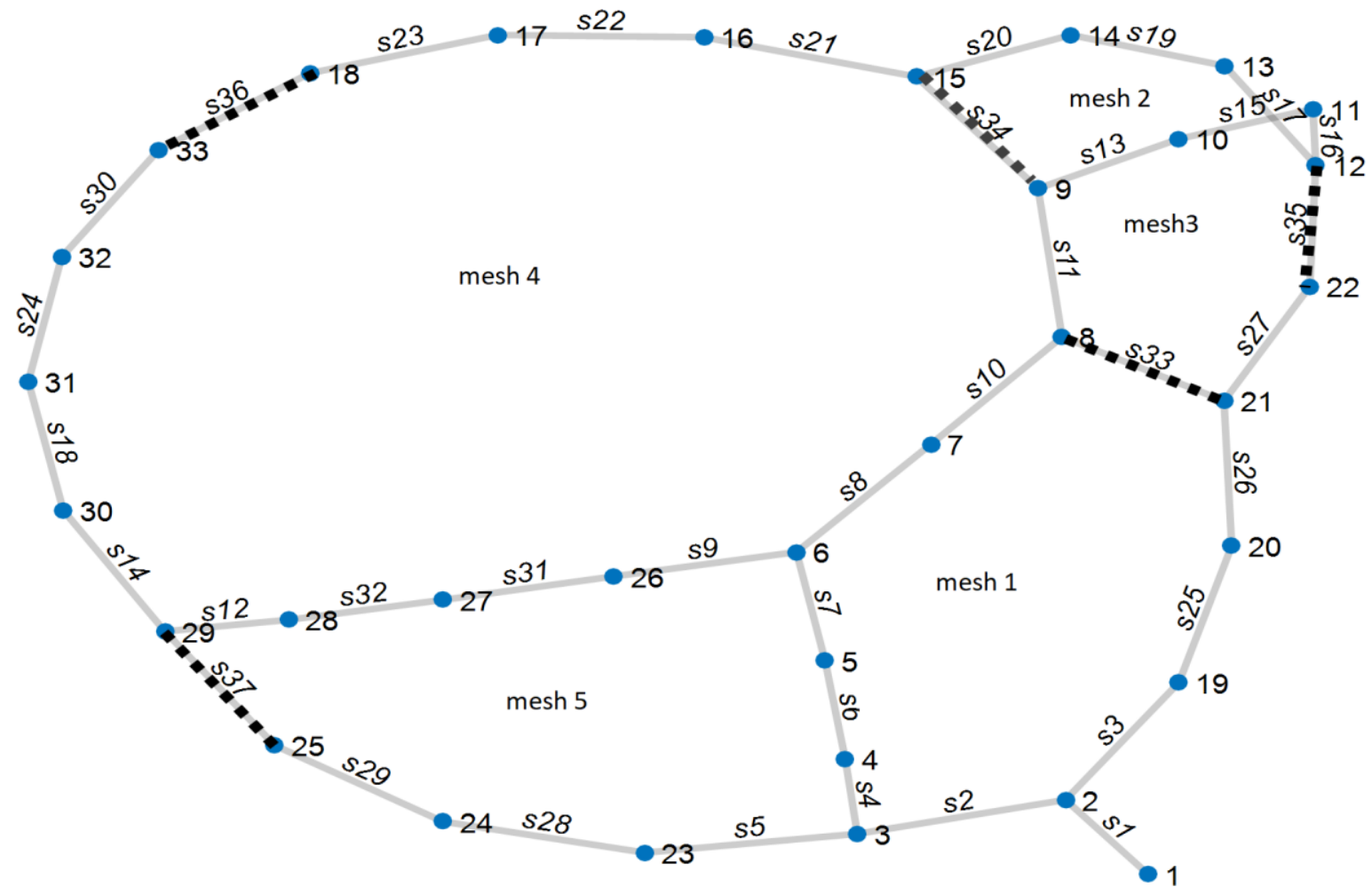

Figure 6.2 Loop representation of a single line diagram of IEEE 33-bus distribution system, where, s denoted switch and the dotted lines represent the tie switches 
Table 6-1 Simulation results of the optimal switching plane and optimal placement and sizing of installed DG units

\begin{tabular}{|c|c|c|c|c|c|c|}
\hline Case\# & \begin{tabular}{|l|} 
DG \\
Location
\end{tabular} & DG size $(\mathrm{kW})$ & $\begin{array}{l}\text { Real } \\
\text { Power } \\
\text { Loss(kW) }\end{array}$ & $\begin{array}{l}\text { Reactive } \\
\text { power } \\
\text { loss(kVAr) }\end{array}$ & $\begin{array}{l}\text { Sub- } \\
\text { current } \\
\text { (KA) }\end{array}$ & $\begin{array}{l}\text { Tie-Switch } \\
\text { Status }\end{array}$ \\
\hline $\begin{array}{l}\text { (Base } \\
\text { Case) }\end{array}$ & - & - & 64.3894 & 54.0280 & 345.1304 & $33,34,35,36,37$ \\
\hline 2 & 33 & 324.8984 & 34.4397 & 26.2894 & 293.1455 & $10,17,27,18,32$ \\
\hline 3 & 5,27 & $\begin{array}{l}\text { 787.5761, } \\
882.4191\end{array}$ & 44.9553 & 32.7910 & 196.2822 & $10,19,27,22,28$ \\
\hline 4 & 19,33 & $\begin{array}{l}683.7605, \\
391.5140\end{array}$ & 38.7450 & 31.7989 & 198.0507 & $6,13,35,18,9$ \\
\hline 5 & $19,33,2$ & $\begin{array}{l}\text { 165.9004, } \\
272.3896, \\
380.0187\end{array}$ & 33.9801 & 26.2634 & 210.8594 & $10,19,27,18,32$ \\
\hline 6 & $\begin{array}{l}2,23,5 \\
24\end{array}$ & $\begin{array}{l}207.9960, \\
568.4179, \\
822.7620, \\
556.3637\end{array}$ & 35.9253 & 27.0175 & 279.2741 & $\begin{array}{l}26,20,35,30, \\
12\end{array}$ \\
\hline 7 & $\begin{array}{l}33,19 \\
23,20 \\
2\end{array}$ & $\begin{array}{l}\text { 533.0945, } \\
913.4328, \\
139.9755, \\
429.4281, \\
821.5341\end{array}$ & 36.7124 & 31.2641 & 218.7507 & $\begin{array}{l}4,19,27,24 \\
32\end{array}$ \\
\hline 8 & $\begin{array}{l}23,2, \\
24,19 \\
22\end{array}$ & $\begin{array}{l}867.5652, \\
813.7468, \\
467.3644, \\
141.2449, \\
738.6867\end{array}$ & 33.0319 & 25.5680 & 313.5803 & $\begin{array}{l}8,15,11,23, \\
31\end{array}$ \\
\hline
\end{tabular}

The most desired objective of the proposed approach is to determine the optimal location and size of the planned DGs units. The real power and reactive power losses, as well as the voltage profile enhancement, are the primary goals of this work. Table 6.1 shows the simulation results of deferent cases for single and multiple DGs unites installed at their optimal location and size. Figure 6,3, and 6.4 presents the simulation results of the case studies. It can be seen, there has been a significant reduction of the real and reactive power 
losses after installing the DGs units and network reconfiguration. The system node voltages are also improved and well constrained between the limits. Feeder current which is the current from the substation also been reduced after installing the DGs units. There has also been a significant achievement in mitigating the node Voltage deviation. The maximum and minimum boundary of the DGs size is set between $50 \mathrm{~kW}$ to $1000 \mathrm{~kW}$. Basically, for each case the installed DGs units inject power to the system and check the node voltages limits if one node violates its limit then the solution will not be considered, and the algorithm will consider another size and location and search for only the feasible solutions where all the constraints are satisfied.
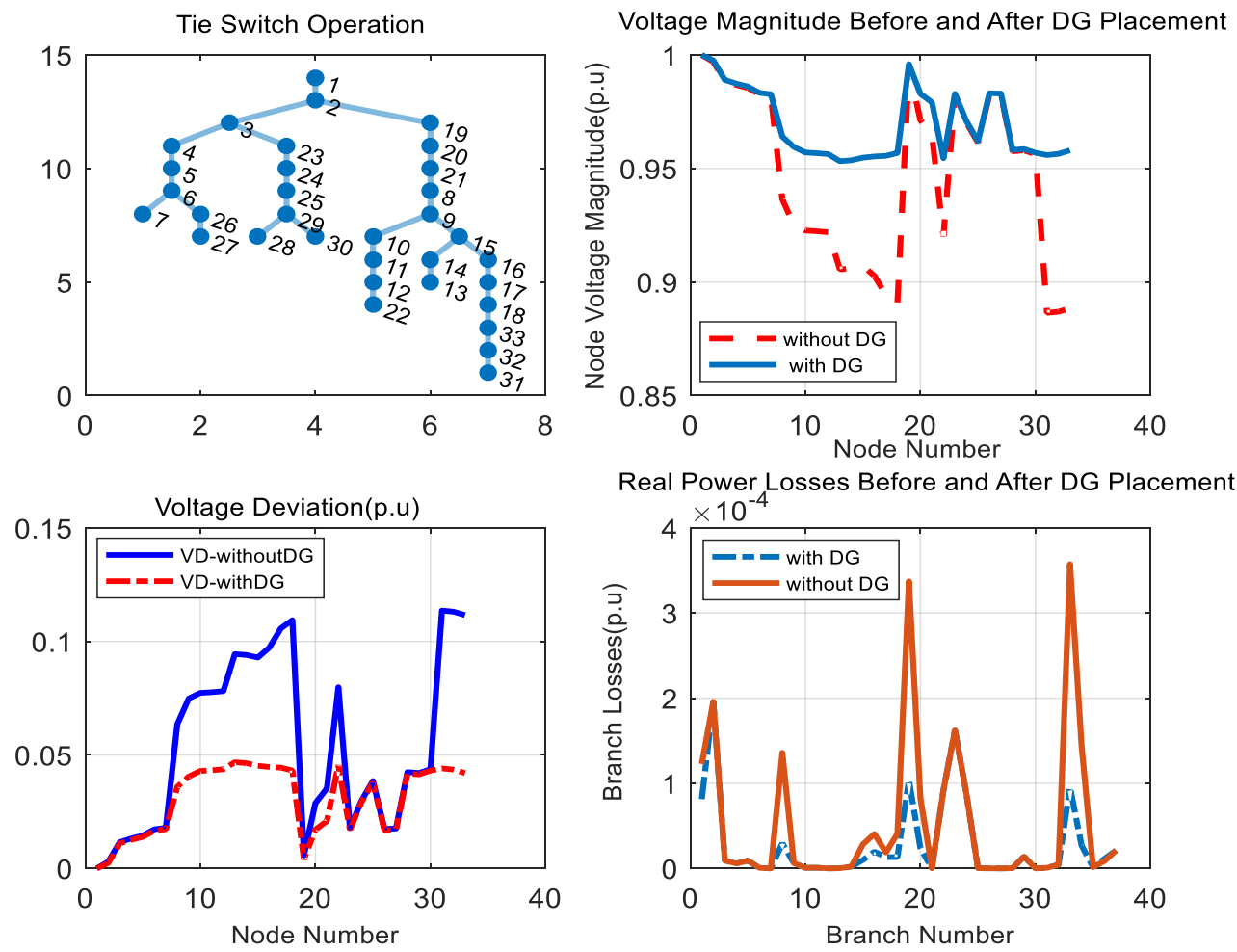

Figure 6.3 Power Flow results with DG unit installed at bus \# 33 

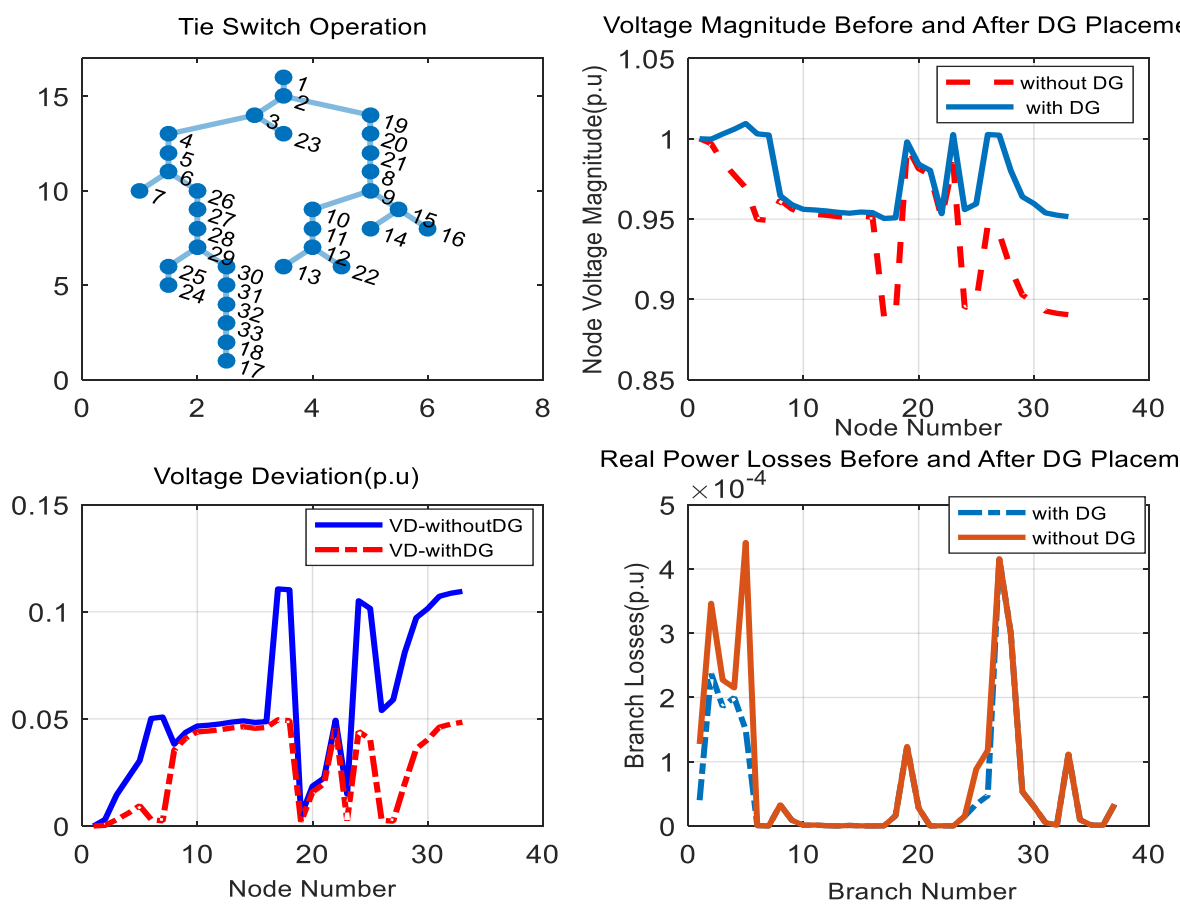

Real Power Losses Before and After DG Placement

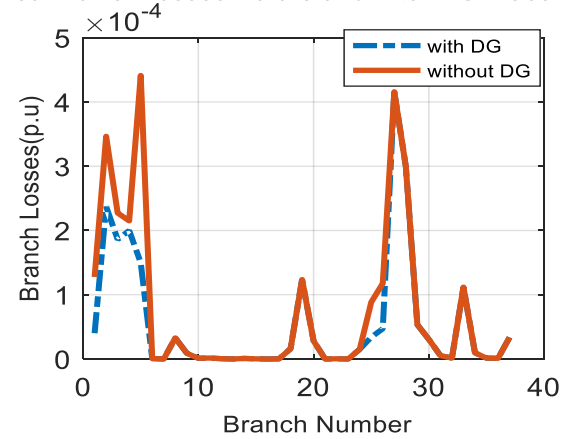

Figure 6.4 Power Flow results with DG unit installed at bus \# 5 and 27
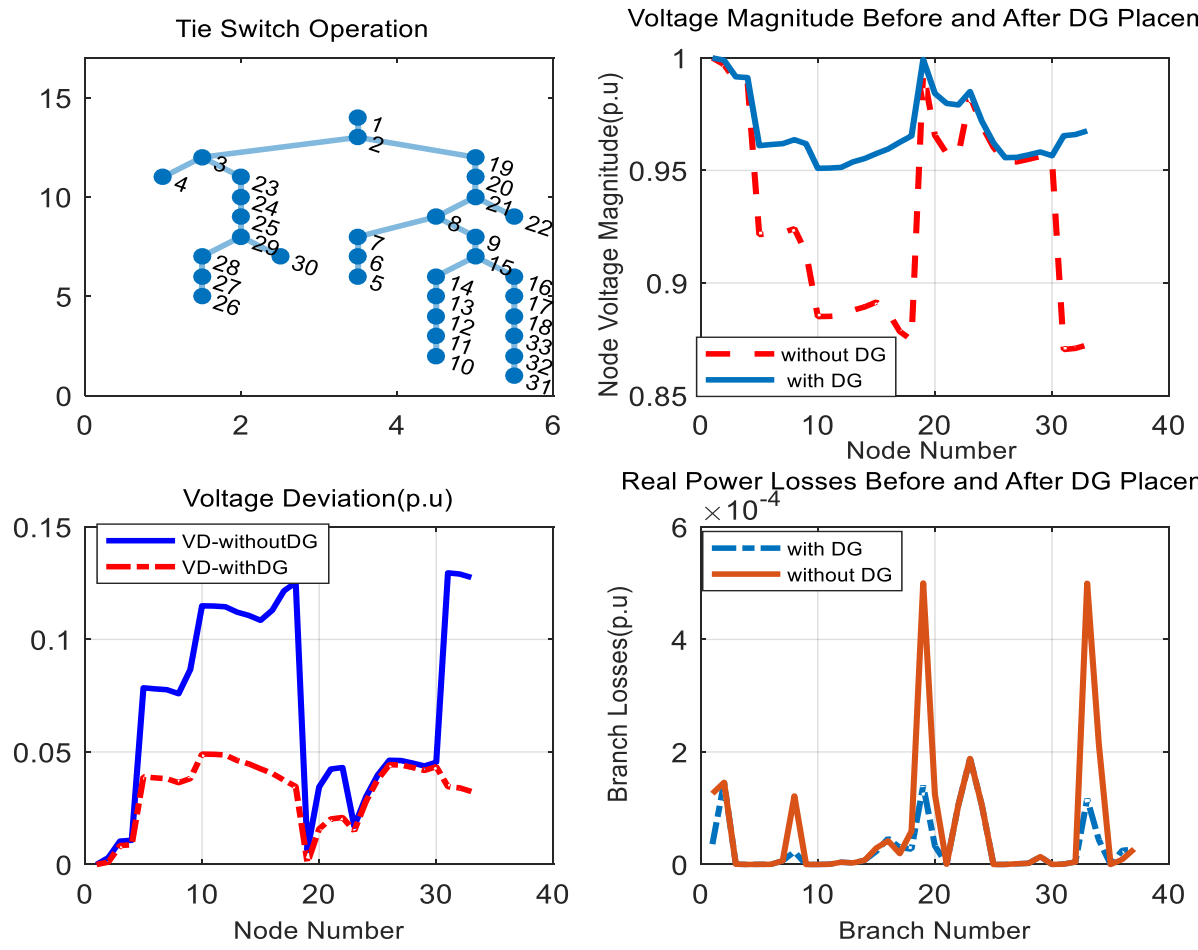

Figure 6.5 Power Flow results with DG units installed at bus \# 19 and 33 

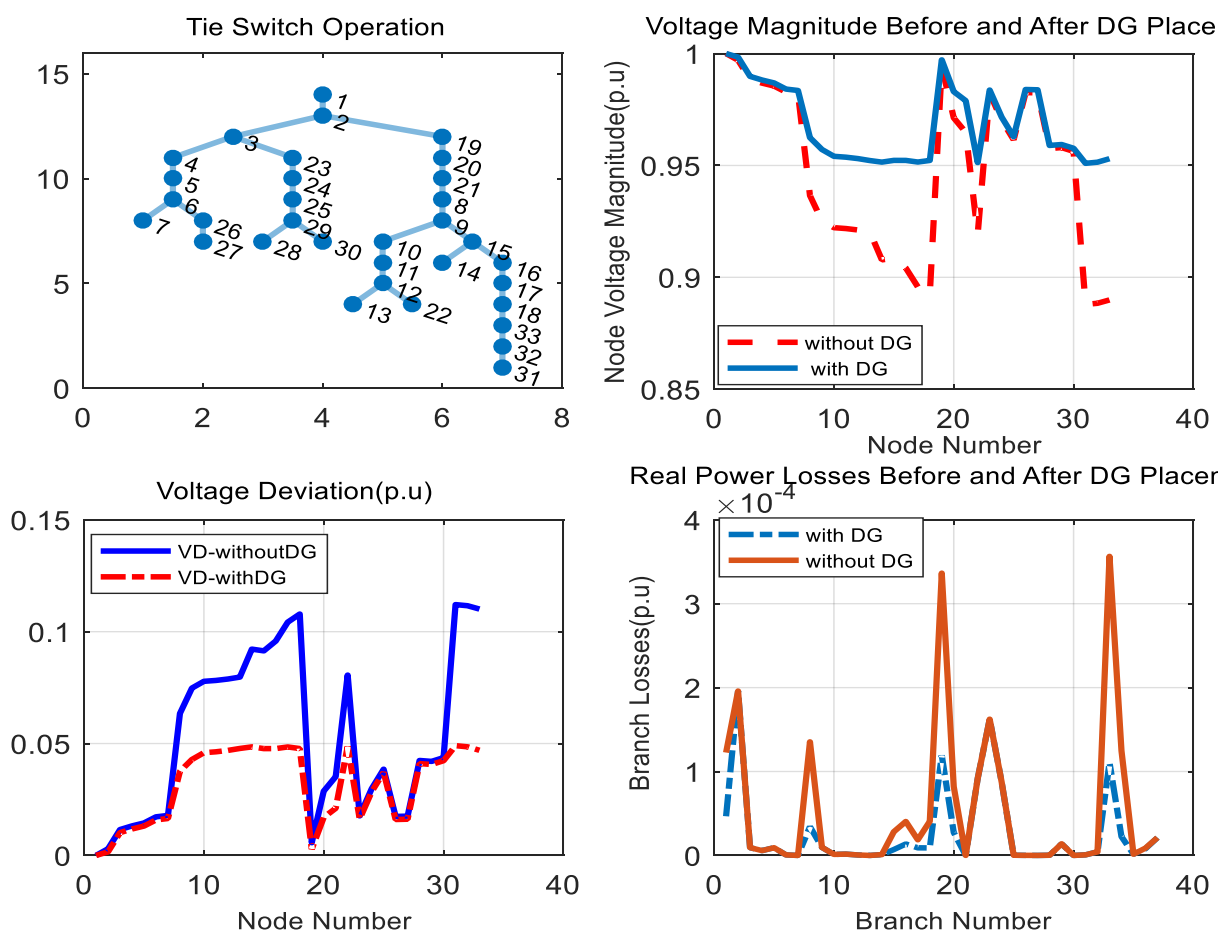

Figure 6.6 Power flow results with DG units installed at bus \# 19, 33 and 2
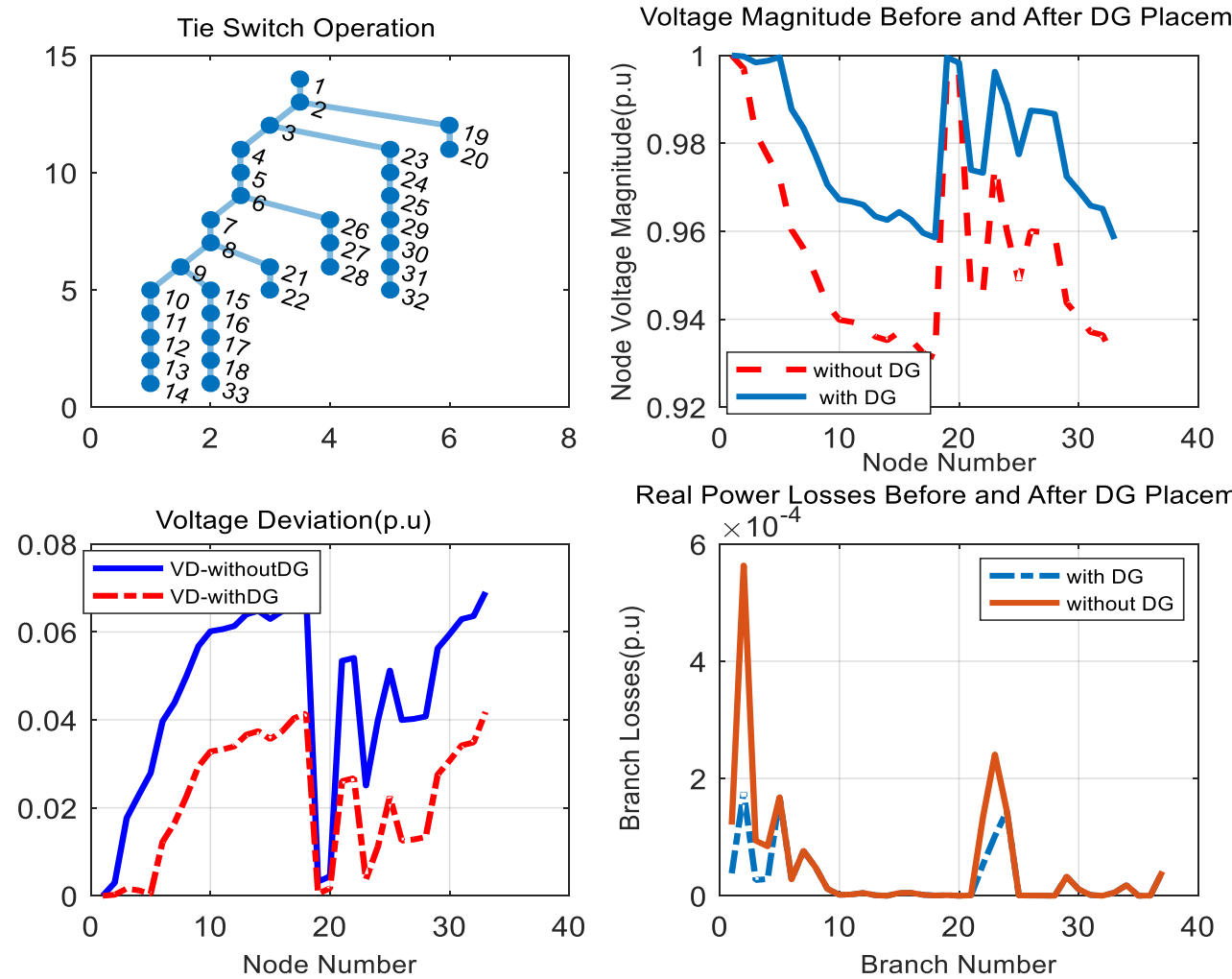

Real Power Losses Before and After DG Placement

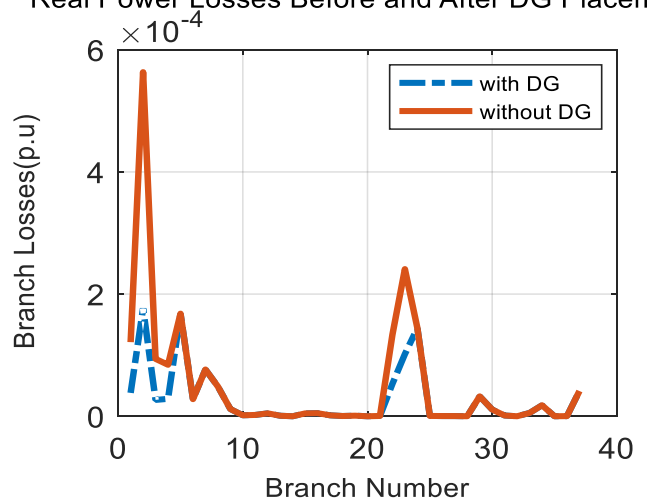

Figure 6.7 Power flow results with DG units installed at bus \# 2, 23, 5 and 24 

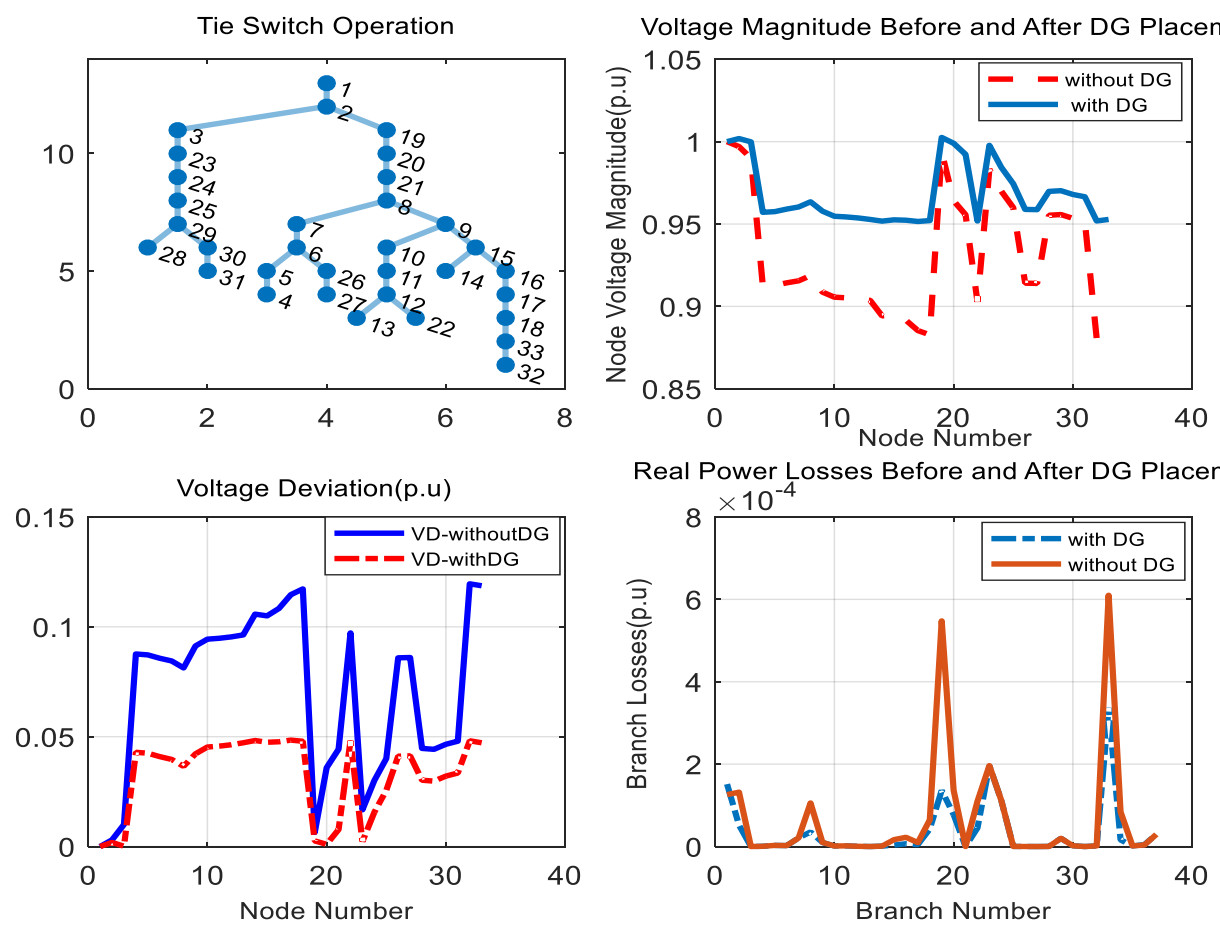

Real Power Losses Before and After DG Placement

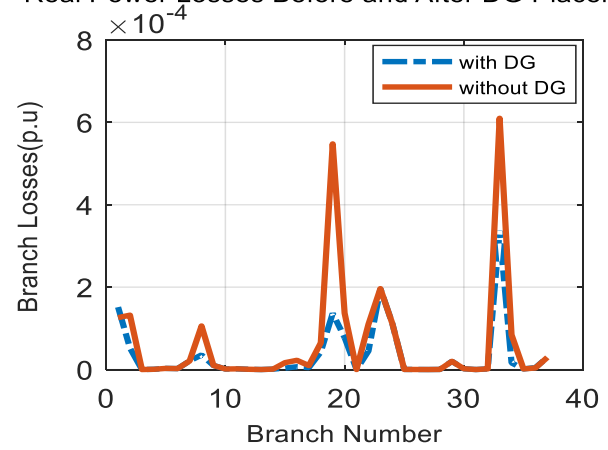

Figure 6.8 Power flow results with DG units installed at bus \# 33, 19, 23, 20 and 2
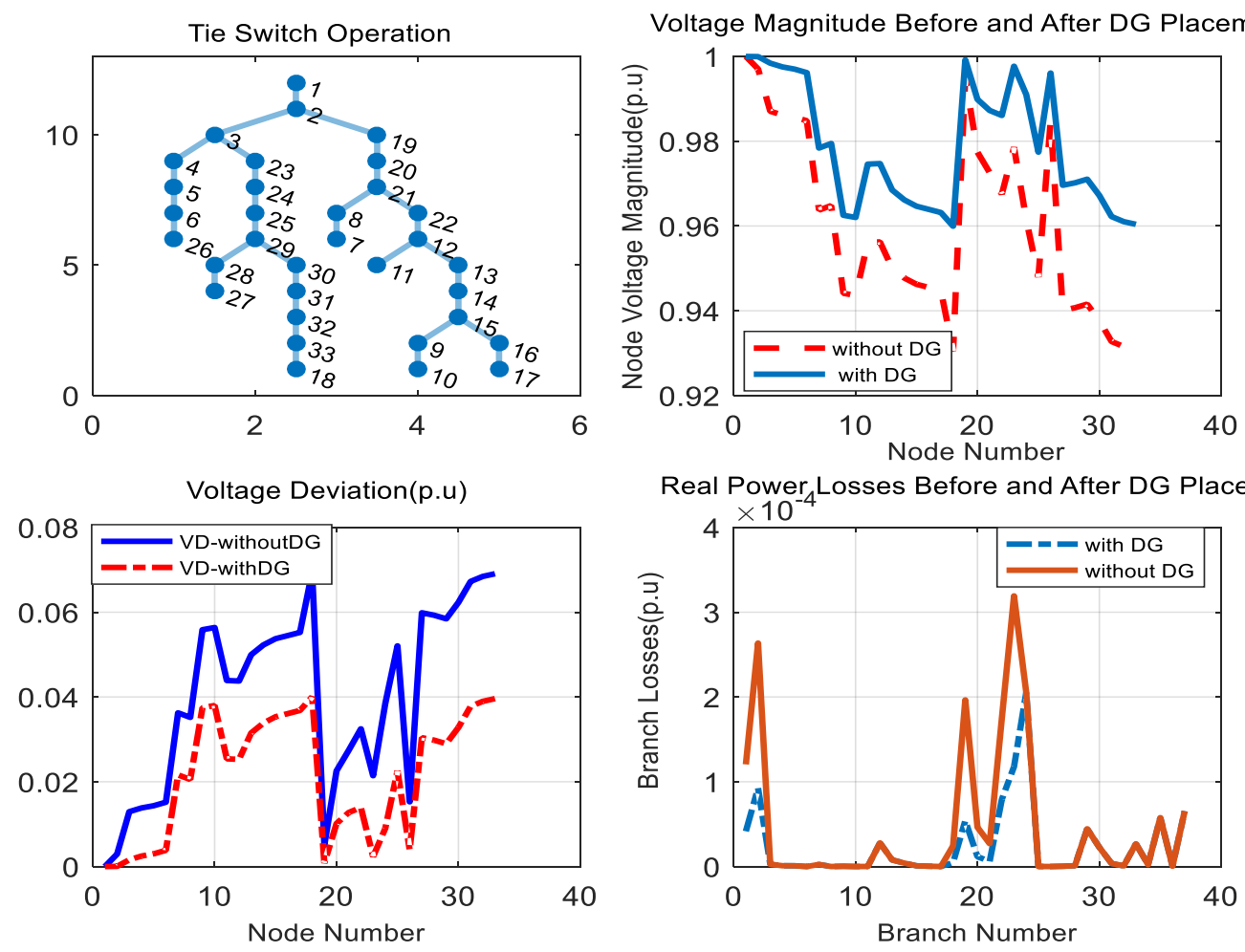

Figure 6.9 Power flow results with DG units installed at bus \# 23, 2, 24, 19, and 22 
Table 6-2 Simulation parameters of DEA

\begin{tabular}{|l|l|l|l|l|l|l|}
\hline $\begin{array}{l}\text { Population } \\
\text { size }\end{array}$ & $\begin{array}{l}\text { Mutation } \\
\text { factor }\end{array}$ & $\begin{array}{l}\text { Crossover } \\
\text { rate }\end{array}$ & $\begin{array}{l}\text { Max } \\
\text { iteration }\end{array}$ & $\begin{array}{l}\text { Number } \\
\text { of } \\
\text { variables }\end{array}$ & $\begin{array}{l}\text { Min DGs } \\
\text { unit } \\
\text { size }(k W)\end{array}$ & $\begin{array}{l}\text { Max DGs unit } \\
\text { size( }(k W)\end{array}$ \\
\hline 100 & 0.8 & 0.7 & 100 & 5 & 50 & 1000 \\
\hline
\end{tabular}

Simulation parameters of the proposed DEA are shown in Table 6.2. Mutation factor and crossover rate are mainly the tuning parameters. The new trial matrix is created from mutation and recombination procedure. Each trial is evaluated, and the obtained results are compared with the ones computed by the corresponding chromosome of the original population matrix. The best fitness is then saved and to be memorized. Figure 6.10 presents the performance of the proposed DEA. From the simulation results, the optimal minima were found in faster time and less iteration. The stopping criteria are that after each evaluation step the size of the population matrix is updated to prevent evaluating the same chromosome more than ones.

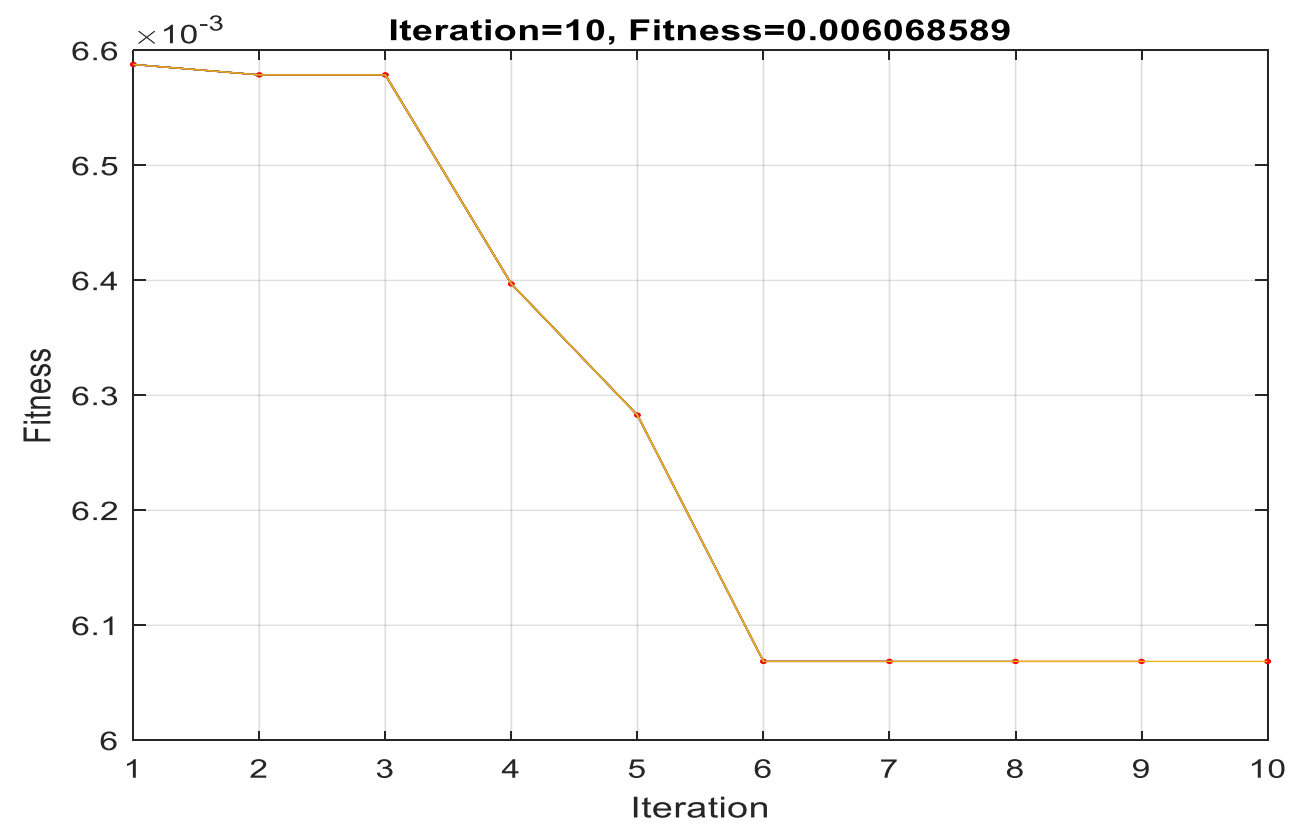

Figure 6.10 Convergence curve of the best fitness value of DE algorithm 


\subsection{Conclusions}

This chapter proposed a new approach for distribution system reconfiguration and optimal sizing and placement of DGs units. The problem is solved by using A Differential Evolution Algorithm DEA. The proposed method in this chapter is a continuation of the method discussed in the previous chapter of optimal distribution feeder reconfiguration with distributed generation using the intelligent technique. The objective of this chapter is to find the optimal location and size of the planned DGs unlike the study of chapter 5 where the size and location were chosen randomly because the assumption was the DGs units already exist in the network. Although, the existing DGs must have the ability to be coordinated and partially controlled by the operator (aggregator), its size and location are still uncontrolled. In this chapter the size of the DGs units was determined using the proposed DEA, and the location was also obtained using sensitivity analysis based on the network real power loss. Simulation results of the IEEE 33-bus test system have demonstrated the efficiency and the effectiveness of the proposed algorithm for determining the optimal sizing and placement of the DGs units and reconfiguring the distribution feeders. The future work would be to investigate the distribution feeder response characteristics, where the hosting capacity of the feeder is to be determined. The hosting capacity is defined as the amount of DERs a feeder can support under its existing topology, configuration, and physical response characteristics. If the hosting capacity is appropriately done, then it will provide a range of information such as, how many DERs can be accommodated without system upgrades, what issues arise at the hosting capacity limits, the location of the DERs so that problems can be avoided, and the location where additional DERs are likely to cause issues on the grid. 


\section{Chapter 7}

\section{Conclusion and Future Work}

\subsection{Conclusion}

In this dissertation, several distribution feeder reconfigurations with and without the impact of the integrated distributed generator have been proposed to investigate and provide a promising solution to reduce the total system power losses and improve the voltage profile. Chapter 1 provided an overview of a distribution system definition. It shows the difference between the traditional distribution system and the smart distribution system besides the impact of the upgrading the existing old networks. The traditional power plant is placed far away from the power consumption area, and electric power is transmitted through long transmission lines. Long transmission lines mean high power loss, less power quality, and lower reliability. Nowadays, the distribution system is neither traditional nor complex system because it has become a smart system. A smart system is a modern form of the traditional power grid which provides more secure, reliable and dependable electrical service. It is, in fact, two-way communication between the utility and the electricity consumer. In addition, the distribution term now is divided into two terms. One-Way distribution in which the power can only be distributed from the central plant using traditional energy infrastructure. The second type is the two-way distribution, while power still provided from the essential power plant, in a smart system, power can bi-directional and flow back to the transmission lines from a secondary power provider.

The review of the literature was presented in chapter 2. It provides the research history of most of the methods and theories applied to the radial distribution system in the effort of mitigation the power losses. it can be noticed that most of the approaches have proposed various topologies to network reconfiguration, and almost all of these studies assumed a balanced network to simplify the computation. However, distribution networks are usually unbalanced due to non-uniform load distribution and nonsymmetrical conductor spacing on the three-phase systems. Additionally, since this research is mainly forcing on optimally solving the optimization problems, the aim was to investigate and study the intelligent technique were used such as genetic algorithm and evolutionary algorithms. 
In chapter 3, distribution feeder reconfiguration for loss reduction was proposed. In this chapter, the problem is formulated as a multi-objective problem considering four objectives related to, minimization of the system real power loss, minimization of the deviations of the nodes voltage, minimization of branch current violation and minimization of feeder's currents imbalance. Since these objectives are different and difficult to be solved by the conventional approaches that may optimize only a single objective. A new approach based on load flow formulation using the graph theory was proposed. It is aiming to formulate and solve the distribution feeder reconfiguration problem using a simple load flow technique for the radial distribution network. This technique involves only the implementation of a simple algebraic expression of receiving-end voltages and employing the graph theory to efficiently construct the system structure. The four objectives are combined through appropriate weighting factors to form the objective function of the radial distribution system as presented in (3.15). Different loss reduction and load balancing scenarios have been investigated. For instance, simulation results study before and after network reconfiguration, with and without including DGs, and after reconfiguration with the presence of single and multiple DG units. At this point, the size and location of the DGs units are selected randomly and it has been assumed as a non-controllable source (owned by the customer). The proposed methods were tested on 70 -node $11 \mathrm{kV}$ and IEEE 33-bus $112.6 \mathrm{kV}$ radial distribution test system. The simulation results have proved the efficiency and feasibility of the proposed method, besides the obtained results has shown high improvement on the system performance by reducing its total power losses and balancing its branch currents. In addition, the convergence characteristics were fast due to the simple algebraic questions used in load flow.

In chapter 4 a new approach based on the Fast-Genetic Algorithm (FGA) was proposed to solve and reconfigure the distribution system problem for power losses minimization. The proposed method is similar to the traditional GA but it defers from in the way of constructing the population matrix. The distribution network is formulated as a meshed system, the number of the meshes are related to the number of open ties. The main idea is relying on setting the boundary of each parameter of the fitness function. There should be no common variable in any boundaries, and also substation nodes and end node load should not be included in the boundaries that are to ensure that all loads are served and system 
radially is retained. Additionally, the proposed method guarantees the fast convergence and comparable results. Most reconfiguration problems are formulated as a mixed integer nonlinear optimization problems. The proposed FGA is dealing with an integer parameter type because the parameters herein is a switch status ( e.g., switch numbers). Integer programming with FGA involves several modifications of the traditional algorithm. It can be noticed from the simulation results that the computed results are satisfied compared with other methods.

In chapter 5, the radial distribution feeder reconfiguration for loss reduction and voltage profile improvement including the presence of DGs using the Differential Evolution DE is proposed. The DGs units are assumed to exist in the system which means its location and size is not controllable since it is a customer owned. DGs can harm the grid if placed or connected randomly resulting in increasing the end user voltage. The study here is aiming to keep the system node voltages within an acceptable limit by configuring the system using DE and checking the voltage violation limit for each load flow execution. The proposed DE algorithm is a new approach used in a power system area. While DE shares similarities with other evolutionary algorithms (EA), it differs significantly in the sense that distance and direction information from the current population is used to guide the search process (e.g., using the current population). In DE algorithm, all solutions have an equal opportunity of being preferred as parents, and selection does not depend on their fitness values. DE performance depends on two processes, known as the mutation process and recombination (crossover) process. The two tuning parameters, called scale factor $(\mathrm{F})$ and crossover probability (CR), which control the performance of DE in its mutation and recombination processes, respectively. The scaling factor, $F \in(0, \infty)$, controls the amplification of the differential variations. The smaller the value of $F$, the smaller the mutation step sizes, and the longer it will be for the algorithm to converge. Larger values for $F$ facilitate exploration, but may cause the algorithm to overshoot good optima. The value of $F$ should be small enough to allow differentials to explore tight valleys, and large enough to maintain diversity. The crossover probability $C R$, has a direct influence on the diversity of DE. This parameter controls the number of elements of the parents that will change. The higher the probability of recombination, the more variation is introduced in 
the new population, thereby increasing diversity and increasing exploration. Increasing $C R$ often results in faster convergence, while decreasing $C R$ increases search robustness.

Chapter 6 proposed a new topology of finding the optimal location and sizing of the planned DG units. The optimizations technique is performed on three different scenarios: reconfiguring the system, finding the optimal locations, and optimal the sizes of the DGs units. In fact, DGs have a great impact on reducing power losses and improving the system performance if placed at its optimal location. However, if DGs placed randomly then they harm the system and cause issues instead of benefits.

The solution methodology presented in this chapter is to evaluate the system whit out DG connection and then reconfigure the system by closing the tie switches and specify the tie with the maximum voltage difference and consider closing this tie first, then the DG location is obtained using the sensitivity of power losses with respect to real power injection at each bus. Then the most sensitive bus is selected for installing the DG unit. Because the integration of the DG adds positive real power injections, the optimal location is the one with the most negative sensitivity in order to get the largest power loss reduction. Finally, after the location is specified the proposed DEA is used to obtain the optimal size of the DG unit. Only the feasible solution who satisfy all the constraint is considered.

\subsection{Future work}

For the optimal location and sizing of planning and integrating DGs units discussed in Chapter 6, which only investigate the optimal connection of the DGs into the distribution system. The distribution performance characteristics have to be taking into account especially with the high interest of energy consumers to install and invest from their DGs. The distribution feeder response characteristics attentional is to determine the hosting capacity of the feeder. The hosting capacity of a feeder is defined as the amount of DERs a feeder can support under its existing topology, configuration, and physical response characteristics. If the hosting capacity is appropriately done then it will provide a range of information such as, how many DERs can be accommodated without system upgrades, what issues arise at the hosting capacity limits, the location of the DERs so that problems can be avoided, and the location where additional DERs are likely to cause issues on the grid. 


\section{References}

[1] D. Burtraw, K. Palmer and M. Heintzelman, "Electricity Restructuring:

Consequences and Opportunities for the Environment," Resources for the Future, Washington, D.C, 2000.

[2] D. A. Augugliaro, "Voltage Regulation and Power Losses Minimization in Automated Distribution Networks by an Evolutionary Multiobjective Approach," in IEEE Transactions on Power Systems, August, 2004, Vol. 19, Issue 3,.

[3] A. P. Engelbrecht, Computational intelligence, South Africa: University of Pretoria, 2007.

[4] D. B. Fogel, "Applying Fogel and Burgin's 'Competitive Goal-Seeking through Evolutionary Programming' to Coordination, Trust, and Bargaining Games.," in In Proceedings of the IEEE Congress on Evolutionary Computation, volume 2 pages 1210-1216,, 2000.

[5] D. Das, "A fuzzy multi-objective approach for network reconfiguration of the distribution system," in IEEE Trans. Power Deliv, vol 21, pp202-209, 2009.

[6] A. a. B. H. Merlin, "Search for a minimal-loss operating spanning tree configuration in an urban power distribution system," in Proceedings of the fifth power system computation conference (PSCC), , Cambridge, UK, 1-18, 1975.

[7] D. H. Shikmoiiammadi, "Reconfiguration of electrical distribution networks for resistive line losses reduction," in IEEE Trans. Power Deliv'., PWRD-4, pp. 14921498, 1989.

[8] D. R. a. R. A. V. Borozan, "Improved method for loss minimization in distribution networks," in IEEE Trans. Power Syst., vol. 10, no. 3, pp. 1420-1425, , Aug. 1995.

[9] J. J. G. H. Y. a. S. S. H. L. S. Civanlar, "Distribution feeder reconfiguration for loss reduction," in IEEE Trans. Power Del., vol. 3, no. 3, pp. 1217-1223,, Jul. 1988.

[10] M. a. W. F. Baran, "Network reconfiguration in distribution systems for loss reduction and load balancing," in IEEE Transactions on Power Delivery, 14011407, 1989.

[11] S. J. L. a. K. V. C. C. Liu, "Loss minimization of distribution feeders: Optimality and algorithms," in IEEE Trans. Power Delivery, vol. 4, no. 1, pp. 1281-1289, Apr. 1989. 
[12] T. Taylor and D. Lubkeman, "Implementation of heuristic search strategies for distribution feeder reconfiguration," in IEEE Trans. Power Del., vol. 5, no. 1, pp. 239-246, Jan. 1990.

[13] H. D. Chiang and R. M. Jean-Jameau, "Optimal network reconfiguration in distribution systems, part 1: "A new formulation and a solution methodology"," in IEEE Trans. Power Del., vol. 5, no. 4, pp. 1902-1909, Oct. 1990.

[14] H. D. C. a. R. M. J.-J. “, "Optimal network reconfigurations in distribution systems, part 2:" Solution algorithms and numerical results"," in IEEE Trans. Power Del., vol. 5, no. 3, pp. 1568-1574, , Jul. 1990..

[15] J. C. K. J. O. K. J. R. S. a. K. Y. L. Y. J. Jeon, "An efficient simulated annealing algorithm for network reconfiguration in large-scale distribution systems," in IEEE Trans. Power Del., vol. 17, no. 4, pp. 1070-1078, Oct. 2002..

[16] ]. C. S. C. a. M. Y. Cho, "Energy loss reduction by critical switches," in IEEE Trans. Power Del., vol. 8, no. 3, pp. 1246-1253,, Jul. 1993.

[17] A. Y. C. a. R. H. T. P. Wagner, "Feeder reconfiguration for loss reduction," in IEEE Trans. Power Del., vol. 6, no. 4, pp. 1922-1933, Oct. 1991.

[18] D. S. a. W. H. E. L. Q. Zhou, "Distribution feeder reconfiguration for service restoration and load balancing," in IEEE Trans. Power Syst., vol. 12, no. 2, pp. 724-729, , May 1997..

[19] D. S. a. W. H. E. L. Q. Zhou, "Distribution feeder reconfiguration for operation cost reduction," in IEEE Trans. Power Syst., vol. 12, no. 2, pp. 730-735, May 1997.

[20] W. M. Lin and H. C. Chin, "A new approach for distribution feeder reconfiguration for loss reduction and service restoration," in IEEE Trans. Power Del., vol. 13, no. 3, pp. 870-875, , Jul. 1998.

[21] V. Borozan and N. Rajakovic, "Application assessments of distribution network minimum loss reconfiguration," in IEEE Trans. Power Del., vol. 12, no. 4, pp. 1786-1792, Oct. 1997.

[22] R. Taleski and D. Rajicic, "Distribution network reconfiguration for energy loss reduction," in IEEE Trans. Power Syst., vol. 12, no. 1, pp. 398-406, Feb. 1997.

[23] N. R. a. S. Sirisumrannukul, "Feeder Reconfiguration for Loss Reduction in ThreePhase Distribution System under Unbalanced Loading Conditions," in IEEE Conv, UPEC 2010, no. 116880603, 1st Aug - 3rd Sept 2010.. 
[24] M.P.Sharma, "Feeder Reconfiguration of Distribution Network Using Minimum Power Flow methodology," in IEEE Conv, India Conference (INDICON), 2015 Annual IEEE, no. 15888391, 17-20 Dec. 2015..

[25] N. a. P. K. Sahoo, "A fuzzy genetic approach for network reconfiguration to enhance voltage stability in radial distribution systems," in Energy Conversion and Management, 47, 3288-3306, (2006.

[26] N. Rugthaicharoencheep, "Feeder reconfiguration with dispatchable distributed generators in distribution system by tabu search," in 44th International Universities Power Engineering Conference (UPEC). Pp 1-5., 2009.

[27] a. M. K. N. G. A. Hemdan, "Distributed generation location and capacity effect on voltage stability of distribution networks," in Student paper annual IEEE Conf., pp. $1-5,2008$.

[28] D. Das, "Distribution Network Reconfiguration using Distributed Generation Unit considering Variations of Load," in IEEE International Conf. Power Deliv, 2016.

[29] F. Ding and K. Loparo, "Feeder reconfiguration for unbalanced distribution systems with distributed generation: a hierarchical decentralized approach," in IEEE Power and Energy Society General Meeting (PESGM), DOI: 10.1109/PESGM.2016.7741124. no. 16463988, 2016.

[30] I. R. a. V. Ganesan, "Coordinated local and centralized control in distribution management systems," in IEEE Trans. Power Del.,vol. 15, no. 2, pp. 718-724, Apr. 2000 .

[31] C. S. C. a. M. Y. Cho, "Energy loss reduction by critical switches," in IEEE Trans. Power Del., vol. 8, no. 3, pp. 1246-1253, Jul 1993.

[32] Y. K. a. M. K. Y. Tzeng, "Generic switching actions of distribution system operation using dynamic programming method," in Proc. IEEE Industrial and Commercial Power Systems Technical Conf, Detroit, MI, USA, 2006, pp. 1-7..

[33] A. S. M. K. a. T. I. K. Nara, "Implementation of genetic algorithm for distribution systems loss minimum re-configuration," in IEEE Trans. Power Syst., vol. 7, no. 3, pp. 1044-1051, , Aug. 1992.

[34] J. a. S. Vasconcelos, "Genetic Algorithm Coupled with a Deterministic Method for Optimization in Electromagnetics," in IEEE Transactions on Magnetics, 3 (2), 1860-1863, 1997.

[35] J. R. J. T. R. a. S. Vasconcelos, "Improvements in Genetic Algorithms," in IEEE Transactions on Magnetics, 37 (5), 3414-3417., 2001. 
[36] J. C. H. a. H. B. Zhang, "Adaptive Probabilities of Crossover and Mutation in Genetic Algorithms Based on Clustering Technique," in Congress on Evolutionary Computation, CEC2004, 2, 2280-2287, 2004.

[37] A. G. J. R. a. F. L. E. Romero, "Path-based distribution network modeling: Application to reconfiguration for loss reduction," in IEEE Trans. Power Syst., vol. 20, no. 2, pp. 556-564, May 2005.

[38] J.-C. Wang and H.-D. Chiang, "An efficient algorithm for real-time network reconfiguration in large-scale unbalanced distribution systems," in IEEE Transactions on Power Systems, Pages: 511 - 517, DOI: 10.1109/59.486141, 1995.

[39] ]. N. Rugthaicharoencheep and S. Sirisumrannukul, "Feeder reconfiguration with dispatchable distributed generators in distribution system by tabu search," in IEEE Universities Power Engineering Conference (UPEC), Proceedings of the 44th International, no. 11, 2009.

[40] S. Ghosh, "Optimal sizing and placement of distributed generation in a network system. Electrical Power and Energy Systems," in International Journal of Electrical Power, Elsevier, 2010.

[41] T. H. M. Gozel, "An analytical method for the sizing and sitting of distributed generators in radial systems. Electrical Power Systems Research," in Electric Power Systems Research, 2009.

[42] A. O. M. Keane, "Optimal Allocation of Embedded Generation on Distribution Networks," in IEEE Transactions on Power Systems, 2005.

[43] M. M. J. Vallem, "Siting and Sizing of Distributed Generation for Optimal Microgrid Architecture," in IEEE Conference, pp. 611-616, 2005.

[44] K. W. O. Wichit, "Optimal Placement of Distributed Generation Using Particle Swarm Optimization'," in M. Tech Thesis., AIT, Thailand , 2005.

[45] M. R. V. U. V. R. N. Lalitha, "Application of fuzzy and PSO for DG placement for minimum loss in radial distribution system," in ARPN Journal of Engineering and Applied Sciences 5(4), 32-37, 2010.

[46] M.-R. F. H. M. O. Haghifam, "Risk-based distributed generation placement," in Transm. Distrib. 2(2), 252-260, 2008.

[47] L. P. F. H. G. Ochoa, "Evaluating distributed time-varying generation through a multiobjective index," in IEEE Trans. Power Delivery 23(2), 1132-1138, 2008. 
[48] G. G. E. M. S. P. F. Celli, "A multiobjective evolutionary algorithm for the sizing and sitting of distributed generation," in IEEE Trans. 20(2), 750-757, 2005.

[49] K. S. M. Vinothkumar, "Impact of DG Model and Load Model on Placement of Multiple DGs in Distribution System," in IEEE Conference, pp. 508-513, 2010.

[50] A. B. Ghaweta and Y. Liao, "Optimal Placement and Sizing of Distributed Generation in a Radial Distribution System Using Differential Evolution Algorithm," in PAC World Americas Conference, Raleigh, NC, 2018.

[51] K. Price, "New Ideas in Optimization," in McGraw-Hill Publishing Company pp. 77-106, 1999.

[52] R. S. J. L. KV Price, Differential evolution: a practical approach to global optimization, Springer, Berlin, 2005.

[53] R. Storn, "Differential Evolution, A Simple and Efficient Heuristic Strategy for Globle Optimization over Continuous Spaces," in Journal of Global Optimization, Vol. 11, Dordrecht, pp. 341-359, 1997.

[54] R. J. Trudeau., "Introduction to Graph Theory," 1993.

[55] Y.T. Hasio and C.Y Chien, "Multiobjective optimal feeder reconfiguration," in IEEE Proc. Genr> Trans. Distrib., vol. 148, pp. 333-336, 2001.

[56] D. Savier, "impact of network reconfiguration on loss allocation of radial distribution system," in IEEE Trans. Power Deliv., vol. 22, pp. 2473-2480, 2007.

[57] M. Guerra, "“Allocation of Distributed Generation for," in Energy Management of Distributed Generation Systems, p. Chapter 12.

[58] "TAB Mittelspannung Technical conditions for connection to the medium-voltage," 2008.

[59] E. Eiríksson, "Distribution grid capability for reactive power support," KTH - Royal Institute of Technology, 2017.

[60] L. Davis, Genetic algorithms and simulated annealing, 1987.

[61] K. S. A. K. M. a. I. T. NARA, "implement of genetic algorithm for distribution systems loss minimum reconfiguration," in IEEE, Trans, Power system, PWRS-7, (3), pp. 1044-1051, 1992.

[62] W. -M. Lin, F. -S. Cheng and M. -T. Tsay, "Distribution feeder reconfiguration with refined genetic algorithm," in IEE Proceedings - Generation, Transmission and Distribution, Pages: 349 - 354, DOI: 10.1049/ip-gtd: 20000715, 2000. 
[63] W. m. lin, F. S. Cheng and M. T. Tsay, "Distribution feeder reconfiguration with refined genetic algorithm," in IEEE proc-Gener.Transm. Distrib. Vol 147.No 6, November 2000.

[64] S. Ghosh and D. Das, "Method for load-flow solution of radial distribution networks," in IEEE Proc-gener.Transm. Distrib.Vol 146, No 6, 1999.

[65] S. Roytelman, "Practical Aspects of Distribution Automation in Normal and Emergency Conditions," in IEEE Trans. on Power Delivery, Vol. 8, pp. 2002-2008, October 1993.

[66] [. S. Kumar, "Memetic Search in Differential Evolution Algorithm," in International Journal of Computer Applications (0975 - 8887), Volume 90- No 6, March 2014.

[67] J. R. and A. C. Sanderson, "Minimal Representation Multisensor Fusion using Differential Evolution," in the International Symposium on Computational Intelligence in Robotics and Automation pages 255-273, 1997.

[68] J. P. Chiou and F. S. Wang, "A Hybrid Method of Differential Evolution with Application to Optimal Control Problems of A Bioprocess System," in the IEEE International Conference on Evolutionary Computation, pages 627-632, 1998.

[69] M. Ali and A. Torn, "Population Set-Based Global Optimization Algorithms: Some Modifications and Numerical Studies," in Computers \& Operations Research 31(10):1703-1725,, 2004.

[70] I. Cruz, v. Willigenburg and v. Straten, "Efficient Differential Evolution algorithms for multimodal optimal control problems," in Applied Soft Computing, 3(2):97-122, 2003.

[71] S. R and P. K. , "Differential Evolution - A Simple and Efficient Heuristic for Global Optimization over Continuous Spaces," in journal of Global Optimization, 11(4):431-359, 1997.

[72] T. Basso, "IEEE 1547 and 2030 Standards for Distributed Energy Resources Interconnection and Interoperability with the Electricity Grid," in National Renewable Energy Laboratory, December 2014.

[73] B. a. J. M. C, "Optimal Placement of Distributed Generators Networks Using Evolutionary Programming," in Proceedings of IEEE PES Transmission and Distribution, Latin American, Venezuela, 2006.

[74] H. Siano, "Strategic Placement of Distributed Generation Capacity," in Proceeding of 19th International Conference on Electricity, Vienna, 2007. 
[75] O. Wichita, "Optimal Placement of Distributed Generation Using Particle Swarm Optimization," in Proceedings of the Australasian Universities Power Engineering, Melbourne, Victoria, Australia., 2006.

[76] A. Hegazy and O. Weerakorn, "A simulated annealing algorithm for multi-objective distributed generation planning," in Australasian Universities Power Engineering Conference - AUPEC, 2010.

[77] Maciel and Padilha-Feltrin, "Distributed generation impact evaluation using a multi-objective Tabu search," in 15th International, Nov. 2009..

[78] G. Vakilian and Ehsan, "A genetic-based Tabu Search algorithm for optimal DG allocation in distribution networks," in Electric Power Components and System, vol. 33, no.12, pp.1351-1363, 2005.

[79] A.-M. and El-Hawary, "Optimal distributed generation allocation and sizing in distribution systems via artificial bee colony algorithm," in IEEE Trans. on Power Del., vol. 26, no. 4, pp.2090-2101, 2011.

[80] B. Zhu, "Impact of DG placement on reliability and efficiency with time-varying loads," in IEEE Trans. Power Syst., vol. 21, no. 1, pp.419-427, 2006.

[81] P. H. Ochoa, "Evaluating distributed time-varying generation through a multiobjective index," in IEEE Transactions on Power Delivery, vol. 23, no. 2, pp.1132-1138, 2008.

[82] Y. L. Ahmad Ghaweta, "Optimal Distribution Feeder Reconfiguration with Distributed Generation using Intelligent Techniques," in ELECTROINFORMATION TECHNOLOGY CONFERENCE, Rochester MI, 2018.

[83] Price, Storn and Lampinen, a practical approach to global optimization, München, Germany: Natural Computing Series, 2005.

[84] S. Kumar, "Memetic Search in Differential Evolution Algorithm," International Journal of Computer Applications, vol. 90 - No 6, pp. 0975 - 8887),, March 2014..

[85] A. Engelbrecht, "Computational intelligence," in an introduction., University of South Africa, Pretoria, Wiley, 2007, p. 241.

[86] F. Ding and K. Loparo, "Feeder reconfiguration for unbalanced distribution systems with distributed generation: a hierarchical decentralized approach," in IEEE Power and Energy Society General Meeting (PESGM), Cleveland , 2016. 
[87] D. Das, "Distribution Network Reconfiguration using Distributed Generation Unit considering Variations of Load," in IEEE International Conf. Power Deliv, (ICPEICES), 2016.

[88] J. H. Teng, "Modeling distributed generations in three-phase distribution load flow," in IET Gener., Transm., Distrib., Vol 2 no.3, pp 330-340, 2008.

[89] L. F. Ochoa and G. P. Harrison, "Minimizing energy losses:optimal accommodation and smart operation," in IEEE Trans. Power syst., Vol 26, no.1 pp 198-205, 2011.

[90] M. Baran, "Network reconfiguration in distribution systems for loss reduction and load balancing," in IEEE Transactions on Power Delivery, 1989.

[91] T. Basso, "IEEE 1547 and 2030 Standards for Distributed Energy Resources Interconnection and Interoperability with the Electricity Grid," National Renewable Energy Laboratory, December 2014.

[92] Benemar and J. M. C, "Optimal Placement of Distributed Generators Networks Using Evolutionary Programming," in Proceedings of IEEE PES Transmission and Distribution, Latin American, Venezuela, 2006.

[93] H. Siano, "Strategic Placement of Distributed Generation Capacity," in Proceeding of 19th International Conference on Electricity, Vienna, 2007.

[94] O. Witchit, "Optimal Placement of Distributed Generation Using Particle Swarm Optimization," in Proceedings of the Australasian Universities Power Engineering, Melbourne, Victoria, Australia., 2006.

[95] Hegazy and Alsharkawy, "A simulated annealing algorithm for multi-objective distributed generation planning," in Proc., 2010. 


\title{
Vita
}

\begin{abstract}
Ahmad B. Ghaweta received the B.Sc. degree in electrical and computer engineering from the University of Almergeb, Alkoms, Libya, in 2003, the M.Sc. degree in electrical engineering from Gannon University, Erie, Pennsylvania, in 2011, and he is currently pursuing the Ph.D. degree in electrical engineering from University of Kentucky, USA.
\end{abstract}

